\title{
Investigation of a Novel NDE Method for Monitoring Thermo-Mechanical Damage and Microstructure Evolution in Ferritic- Martensitic Steels for Generation IV Nuclear Energy Systems
}

\section{Reactor Concepts RD\&D}

\author{
Dr. Peter Nagy
} University of Cincinnati

In collaboration with: Idaho National Laboratory 
NEUP 10-849

Investigation of a Novel NDE Method for Monitoring Thermo-Mechanical Damage and Microstructure Evolution in Ferritic-Martensitic Steels for Generation IV Nuclear Energy Systems

Workscope G4A-2

\title{
Final Report
}

\author{
Peter B. Nagy ${ }^{1}$, Vijay K. Vasudevan ${ }^{2}$, Dennis C. Kunerth ${ }^{3}$ \\ ${ }^{1}$ Department of Aerospace Engineering and Engineering Mechanics \\ University of Cincinnati \\ peter.nagy@uc.edu \\ ${ }^{2}$ Department of Mechanical and Materials Engineering \\ University of Cincinnati \\ vasudevk@ucmail.uc.edu \\ ${ }^{3}$ Materials Science and Engineering Department \\ Idaho National Laboratory, \\ dennis.kunerth@inl.gov
}

September 30, 2013 


\section{Contents}
Abstract

Acknowledgements

1. Introduction

2. Directional Potential Drop Measurement for In Situ Creep Monitoring

2.1 Geometrical gauge factor

2.2 Material gauge factor

2.3 Oriented clusters of microcracks

2.4 Inversion for creep under uniaxial stress

2.5 Inversion for creep under biaxial stress

3. Directional Potential Drop Measurement for Manual Creep Characterization 3.1 Deployable DACPD probe

3.2 Apparent anisotropy of a normal boundary between two conducting domains

3.3 Numerical validation of the analytical predictions

3.4 Boundary inclination and gradual transition

4. DACPD Creep Assessment with Deployable Electrodes

4.1 Effect of thermal evolution of the microstructure

4.2 Electric properties of $9 \mathrm{Cr}$-1Mo steel

5. DACPD Creep Monitoring System with Permanently Installed Electrodes

5.1 First generation DACPD system and validation tests

5.2 System validation

5.3 Second generation DACPD system

5.4 Creep tests with DACPD monitoring

5.5 Creep tests in welds

5.6 Creep tests in Gleeble thermo-mechanical simulator at INL

6. Metallurgical Investigation

6.1 Inspection methods

6.2 Inspection results and discussion

7. Conclusions 


\section{Abstract}

The main goal of this project was the development and validation of a novel nondestructive evaluation (NDE) technique for in-situ monitoring of ferritic-martensitic steels, like Grade 91 9Cr-1Mo, which are candidate materials for Generation IV nuclear energy structural components operating at temperatures up to $\approx 650{ }^{\circ} \mathrm{C}$ and for steam generator tubing for sodium-cooled fast reactors. Full assessment of thermo-mechanical damage requires a clear separation between thermally activated microstructural evolution and creep damage caused by simultaneous mechanical stress. This research project utilized a multifaceted approach in which the feasibility of low-frequency electrical NDE methods was studied and coupled with detailed postthermal/creep exposure characterization of microstructural changes and damage processes using state-of-the-art electron microscopy techniques. Our aim was to establish the most effective nondestructive materials evaluation technique for particular degradation modes in Grade 91 steel as well as to provide the necessary mechanism-based underpinnings for relating the two.

Microstructure evolution and cavity nucleation and growth over the creep life of 9Cr-1Mo ferritic-martensitic steel were investigated. The microstructure of the samples was characterized by transmission electron microscopy, electron backscattered diffraction microscopy, and scanning electron microscopy to determine the accurate grain size, boundary character, texture formation, and precipitation characteristics. The results show that at high stress levels damage initiation and failure happen in a very short time, whereas at lower stress levels damage initiation and propagation are distinguishable before fracture.

Our NDE development effort was based on the directional alternating current potential drop (DACPD) technique using a square-electrode configuration. This measurement technique is based on a modified potential drop (PD) technique and measures simultaneously two resistance values in orthogonal directions. Our tests showed that small anisotropic changes in the two resistances caused by directional effects of creep can be distinguished from potentially far larger isotropic changes caused by non-directional reversible and irreversible thermal effects. The sensitivity of the square-electrode PD sensor to geometrical and material variations was analyzed separately and the analytical predictions were validated by experimental results. The directional PD technique was found to exhibit high sensitivity that allows the detection of both elastic and plastic strains. Extensive creep tests lasting between 4 hours and 4,000 hours were conducted in Grade $919 \mathrm{Cr}-1 \mathrm{Mo}$ steel between $70 \mathrm{MPa}$ and $150 \mathrm{MPa}$ at $650^{\circ} \mathrm{C}$.

Our tests with permanently mounted electrodes demonstrated the feasibility of the DACPD technique for creep to monitoring. Permanently mounted probes are ideal for both continuous and intermittent creep monitoring since the electrodes are attached to the surface and follow the deformation of the component, therefore the measured resistance ratio is sensitive not only to material changes caused by creep damage but also to much stronger geometrical changes caused directly by creep strain. However, in some applications, it is desirable to use deployable probes even if the sensitivity to creep is necessarily lower. Therefore, we also developed a deployable DACPD probe that is solely sensitive to material effects and can be used to detect creep-induced electric anisotropy in the material by manually scanning the surface area of interest.

Through the results obtained from this integrated materials behavior and NDE study, new insight was obtained into the dominant creep degradation mechanism in Grade 91 steel. The project also included collaboration with partners at the Idaho National Laboratory and our results will serve as a foundation to guide further efforts of scientists at industrial, government and university laboratories concerned with monitoring creep and microstructural evolution in materials planned to be used in Generation IV Nuclear Energy Systems. 


\section{Acknowledgements}

This work was supported by the Nuclear Energy University Program (NEUP) of the US Department of Energy subcontract 00101721 issued under prime contract DE-AC07-05ID14517 to Battelle Energy Alliance, LLC. The authors would like to express their appreciation to their graduate students, Elhoucine Madhi, Seeran Prajapati, Nataraj Jonnalagadda, William Banks, Xiaoka Xinag, Don Carraher, and Deepthi Tammana, who participated in this project and provided valuable contributions to this report. We are especially grateful to Senior Research Associate Curtis Fox for his invaluable contributions to measurement automation, and hardware and software development and to Postdoctoral Fellow Dr. Behrang Poorganji for his valuable work on metallurgical characterization of our specimens.

We would also like to thank Professor Leijun Li of the Department of Mechanical and Aerospace Engineering of Utah State University for providing a large piece of welded G91 steel pipe for our investigation. We are very grateful to the Idaho National Laboratory for providing a large quantity of G91 9Cr-1Mo steel material for our study and in particular to Dr. Corrie I. Nichol of Idaho National Laboratory who helped us adapt the directional alternating current potential drop creep monitoring system to work with the Gleeble thermo-mechanical simulator of the Idaho National Laboratory and conducted a series of preliminary tests described in this report. Finally, we would like to thank Chris Ward of RWE npower for providing some of the creep damaged specimens and accompanying replica results described in this report. 


\section{Introduction}

Ferritic-martensitic steels derive their creep strength from solid solution strengthening, precipitation hardening through complex inter- and intragranular metal carbonitride particles and from the phase-transformation induced dislocation sub-structures [1]. These strengthening mechanisms are vulnerable to in-service degradation due to thermal exposure and creep, which takes the form of extensive deformation and cracking and may compromise the material's safe long-term operation, thus necessitating costly inspection and repair. A number of NDE methods with potential monitoring capability have been identified in previous studies and electromagnetic methods have emerged as the leading candidates for such applications because of the relative ease of their adaptation for continuous monitoring of subtle microstructural changes under field conditions [2]. In order to optimize the inspection procedure, the sensitivity and selectivity of individual techniques must be studied for different types of damage mechanisms on a case-tocase basis.

It should be noted that the operating characteristics of GEN IV high temperature structural components involve long service lives ( $>60$ years). As it is not often practical to perform longterm creep tests under these conditions and obtaining creep-fatigue test data required to determine the negligible creep condition is very challenging because of the long test times that are involved, it is a prudent strategy to compensate for the high uncertainty in the negligible creep conditions by implementing NDE procedures to achieve better health/safety management of nuclear power plants. We conducted an investigation of the feasibility of a novel NDE method that is capable of reliable, in-situ monitoring of creep damage and changes in microstructure of materials and components in the field.

During creep deformation, material damage accumulates with time as a function of temperature and mechanical stress. The specific form of degradation is material dependent, but in creep-resistant steels it might follow two main types of path [3]. First, there is transformation and precipitation of carbides from the initial microstructure. These precipitations might be in the form of tempered martensite and bainite or pearlite and ferrite, depending on the heat treatment used. Second, at a later stage of the degradation process, the presence of voids and microcracks becomes more evident. For creep-resistant materials used at high temperatures, creep rupture caused by cumulative damage is a common failure mode. The process starts with the nucleation and then growth of cavities at grain boundaries. The cavities tend to gather preferentially on grain boundaries approximately perpendicular to the applied stress [4-6]. Eventually these cavities connect together to form microcracks that propagate and join together in a later stage of degradation that leads to ultimate failure. This process leads to anisotropic texture in electric resistivity, which can be exploited for creep monitoring. In effect, the resulting preference in damage orientation introduces small but perceivable path-length differences for electrical current. This induced anisotropy is detectable by directionally sensitive sensors.

Most damage detection techniques have sufficient level of sensitivity for the purposes of damage monitoring, but the crucial issue is their selectivity, or the lack of it, to a specific material degradation mechanism. There are numerous variables to which a given sensor can be sensitive including microstructure evolution, carbide precipitation, hardening, plastic strain and elastic strain. Effective creep monitoring requires that the sensor sufficiently suppress changes that are primarily associated with thermally activated microstructural evolution. Even prior to mechanical loading, the material contains various microdefects, such as microcracks, voids, inclusions, and second-phase particles [7]. Also, most steel components used in power plants accumulate an initial amount of cold work during manufacturing. The presence of cold work induced texture and directional defects is expected to result in an initial material anisotropy that 
is detectable by sensitive probes as recovery and relaxation takes place during thermal exposure.

Because of the above described complexities, creep monitoring in general and detection of void precursors in early stages of creep in particular continue to be great challenges and also great opportunities for the nondestructive evaluation community. The most popular techniques for creep detection include replica metallography, various forms of surface strain measurements, ultrasonic velocity, attenuation, backscatter, and birefringence measurements, magnetic methods including Barkhausen and magneto-acoustic emission, hardness measurements, positron annihilation, small-angle neutron scattering, eddy current and various other electromagnetic methods. For a critical comparison of the most important techniques see the recently published comprehensive review by Sposito et al. [2]. From the point of view of the present project, efforts to use electric potential drop (PD) measurements are the most relevant. PD assessment of creep damage was found especially promising early on in the degradation process [8,9]. PD creep assessment exploits changes in electrical resistivity due to microstructural evolution and damage accumulation throughout the creep life of the material [10]. Several papers have reported that long-term thermal exposure of low-alloy ferritic steels causes a perceivable reduction in electrical resistivity and that simultaneous applied stresses lead to even greater reductions [9-21]. It was reported that during creep the electric resistivity tends to first decrease until about $40 \%$ of creep life [11]. Around this point, the resistivity stabilizes and eventually starts to increase as failure approaches. In summary, these recent studies indicate that PD techniques are well suited for monitoring creep degradation and that their main advantage over alternative inspection methods is their ability to monitor creep in early stages when most other NDE methods cannot yet detect the resulting damage [2]. Unfortunately, microstructural evolution, which is not necessarily creep-related, also contributes and sometimes dominates changes in electric resistivity [15].

In recent years, electric resistivity measurement has emerged as a leading candidate for monitoring microstructural changes caused by thermal exposure in steel components. Under creep loading, part of the increased resistivity is due to increasing porosity, but microstructural evolution also contributes to the change in resistivity. In this context, the key microstructural changes that occur in ferritic-martensitic steels, like modified $9 \mathrm{Cr}-1 \mathrm{Mo}$, which have enormous influence on the creep properties and rupture life, include recovery of the sub-grain/dislocation structure within the tempered martensite laths and precipitation of Fe2M Laves phase and carbides/carbonitrides of the $\mathrm{MX}(\mathrm{VN}, \mathrm{NbC})$ and $\mathrm{M} 23 \mathrm{C} 6$ types [1]. The sequence in which these features evolve with time is a strong function of applied stress and test temperature. The above studies suggested that electrical resistivity monitoring can be used for the measurement of volumetric creep damage, especially at early stages when creep damage is hardly detectable by other methods. All previous efforts directed at creep damage assessment by ACPD relied on non-directional resistance measurements using conventional in-line electrodes. In the following section we will lay down the theoretical foundation of directional alternating current potential drop measurements as a viable alternative. When used with permanently attached electrodes, the method is mainly sensitive to geometrical effects of strain, but in the final stage of creep it is also sensitive to the creep-induced electric anisotropy of the material.

\section{Directional Potential Drop Measurement for In Situ Creep Monitoring}

In this project we exploited an advanced directional alternating current potential drop (DACPD) technique to achieve the high inspection sensitivity needed to monitor gradual material degradation and microstructural changes in both base metal and welded structures of ferriticmartensitic GEN IV steels like modified 9Cr-1Mo. To aid interpretation of the data, state-of-the- 
art instrumentation and advanced digital signal processing methods were used. A robust understanding of the relationship between the mechanical and electromagnetic properties of the material with its microstructure at the nano- and micro-scales was also developed. Throughout this project, special attention was paid to the necessity of developing a simple, reliable, and rugged nondestructive monitoring procedure that can be easily adapted to field conditions. This ultimate goal determined our approach of screening the most adaptable NDE techniques for sensitivity and selectivity rather than starting with the most sensitive and selective known laboratory techniques and later trying to adapt them to in-situ monitoring. Ideally, such sensors should have a low profile, be of robust construction, be unaffected by the operating environment and have simple wire connections. Electromagnetic methods are well suited for this purpose. Conventional alternating current potential drop (ACPD) methods have an established track record in nondestructive characterization of material properties in a wide range of engineering materials and are particularly well suited for monitoring thermo-mechanical damage.

Most PD techniques measure the electrical transfer impedance of a conducting material with a pair of injection electrodes and a separate pair of sensing electrodes arranged in a line as shown in Figure 1. In the so-called direct current (DC) or quasistatic mode the spread of the injected current inside the specimen is controlled by geometrical effects and the distribution of the electric resistivity of the material. Similar alternating current (AC) measurements can be conducted at sufficiently high frequencies so that the frequency-dependent electromagnetic "skin" depth becomes first comparable and then smaller than the quasistatic penetration depth of the current distribution in the specimen. Because of this, ACPD measurements offer better control over the inspected depth in the specimen and yield larger, therefore more easily measurable, potential differences at lower levels of injection current. Furthermore, ACPD measurements can exploit phase-locked detection to increase the measurement accuracy. In order to minimize the spurious effects of large variations in magnetic properties, in this study we consider only low-frequency, essentially quasistatic, ACPD measurements when the skin effect is negligible and the injection current spreads throughout the whole cross section of the specimen limited only by the electrode separation just like in the case of DCPD measurements. For a comprehensive summary of the use of four-point potential drop measurements for materials characterization purposes see the recent review by Bowler [22].

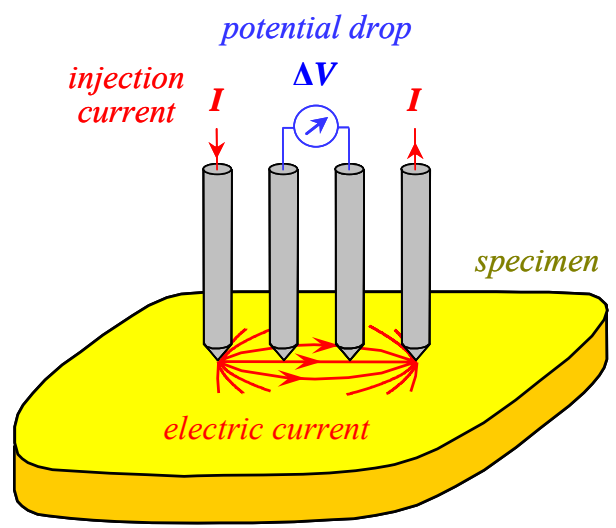

Fig. 1 A schematic diagram of a typical PD measurement with in-line electrode configuration.

It was recently suggested that directional PD resistance measurements with square-electrode configuration offer many advantages over the conventional non-directional in-line electrode 
configuration in creep monitoring [23]. A permanently installed sensor consists of the material to be tested and four thermocouple wires spot-welded to it as shown schematically in Figure 2. Let us assume that a uniaxial tensile load is applied in the $x_{1}$ direction. First, the "axial" transfer resistance $R_{1}=V_{1} / I_{1}$ is obtained by injecting current $I_{1}$ at electrodes A-B and measuring the resulting in-phase voltage drop $V_{1}$ between electrodes D-C. Then, the "lateral" transfer resistance $R_{2}=V_{2} / I_{2}$ is obtained by injecting current $I_{2}$ at electrodes A-D and measuring the resulting in-phase voltage drop $V_{2}$ between electrodes B-C. Creep can be monitored through the variations observed in the resistance ratio $R_{1} / R_{2}$. It should be mentioned that, based on the Reciprocity Theorem, the injection and sensing electrodes can be exchanged without affecting the measured transfer resistances, which might be exploited to improve the measurement accuracy.
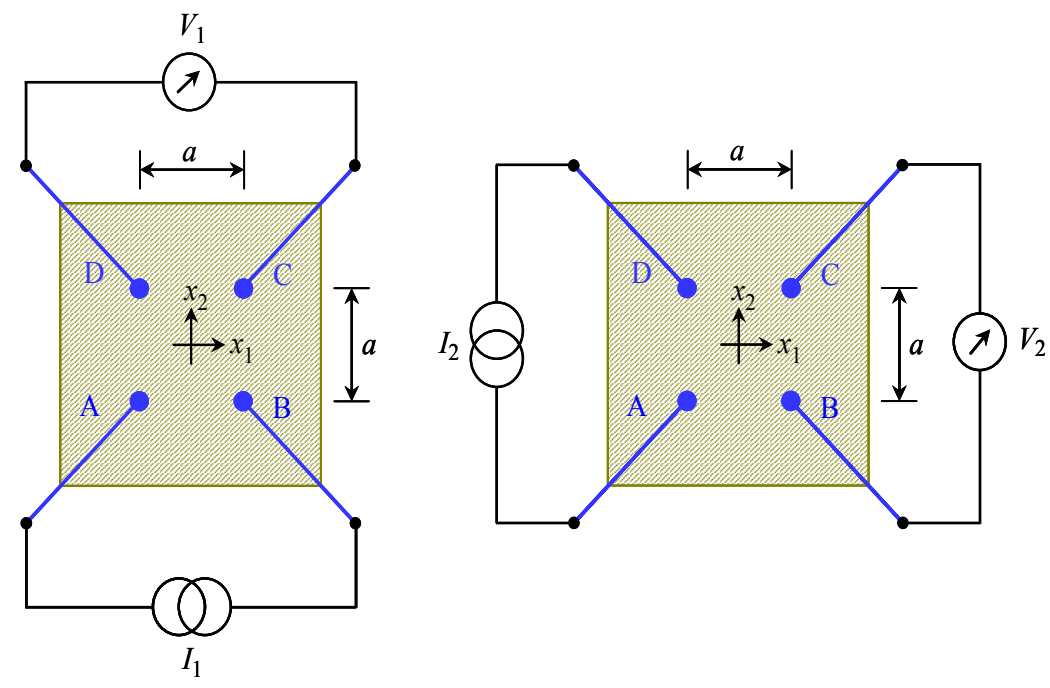

Fig. 2 A schematic diagram of directional PD measurement with square-electrode configuration.

Generally, the measured electric resistances $R_{1,2}$ are complicated functions of both reversible and irreversible thermal, mechanical and thermo-mechanical (creep) effects in the material. For our current purposes, these effects can be modeled as follows

$$
R_{1,2}(T, \varepsilon) \approx R_{10,20} B_{1,2}(T) C_{1,2}(\varepsilon),
$$

where $R_{10}$ and $R_{20}$ are the initial "intact" values of $R_{1}$ and $R_{2}$, respectively, $B_{1} \approx B_{2}$ represent the very similar temperature dependence of the resistance in the two principal directions, $T$ is the instantaneous temperature of the specimen, $C_{1,2}$ are functions that represent the strain dependence of the resistances in the principal directions, and $\varepsilon$ is the total axial strain that includes both elastic $\varepsilon_{\mathrm{e}}$ and plastic $\varepsilon_{\mathrm{p}}$ strain components. Since thermal expansion is negligible in comparison with the much higher temperature dependence of the electric resistivity, $B_{1,2}(T)$ can be approximated as a purely material effect

$$
B_{1,2}(T) \approx 1+\beta\left(T-T_{0}\right)^{m}+\beta_{1,2},
$$

where $\beta$ and $m$ are the essentially isotropic coefficient and power of reversible temperature variation, respectively, $T_{0}$ is an arbitrary reference temperature, and $\beta_{1,2}$ represent the irreversible 
changes caused by thermally-activated microstructural evolution. These irreversible thermal effects are essentially identical, but might exhibit slightly different values in the $x_{1,2}$ directions partly because of preexisting texture and residual stress in the "intact" material and partly because of possible directional thermally-activated microstructural changes in the presence of unidirectional state of stress.

In order to suppress inevitable variations in electrode positioning and other inherent variations unrelated to subsequent creep, such as preexisting inhomogeneity and texture that together could result in as much as $10-20 \%$ difference between $R_{10}$ and $R_{20}$, the measured resistances are first normalized to their initial intact values

$$
\eta_{1,2}=\frac{R_{1,2}(T, \varepsilon)}{R_{10,20}},
$$

where the normalized resistances $\eta_{1,2}$ are functions of temperature and strain. In the second step of data evaluation the normalized resistance ratio is calculated between the axial and lateral directions as follows

$$
\xi=\frac{\eta_{1}(T, \varepsilon)}{\eta_{2}(T, \varepsilon)} .
$$

This second normalization is necessary because during creep the reversible resistivity changes over a typical service temperature range of $500-700{ }^{\circ} \mathrm{C}$ for power plant steels often exceed 100 $200 \%$ while the expected overall increase of $\xi$ in the early stages of creep is only a few percent. Fortunately, this strong reversible temperature effect is mainly isotropic since the increase in lattice vibrations at high temperature causes the resistivity to increase by equal amounts in every direction, which was exploited in Eq. (2).

In contrast to the reversible temperature effects, the irreversible resistivity changes $\beta_{1,2}$ caused by thermal exposure are usually not entirely isotropic. As we mentioned earlier, any electric anisotropy caused by pre-existing texture in the microstructure or stress relaxation can cause directional irreversible resistivity changes due to thermal exposure. Thermally-activated microstructural evolution starts at significantly lower temperature and progresses at significantly higher rate in the presence of microstructural damage [24]. For instance, in cold-rolled materials resistivity changes with thermal exposure can be notably different in two principal directions. This difference in resistivity variation with thermal exposure introduces apparent directionality to the measurement since the orthogonal resistances are normalized to their respective initial values which include the effects of preexisting anisotropic texture.

The fact that the temperature effect $B_{1,2}(T)$ is mostly isotropic while the strain effect $C_{1,2}(\varepsilon)$ is highly anisotropic can be exploited for the separation of these two competing effects

$$
\xi=\xi_{\mathrm{g}} \xi_{\mathrm{m}} \approx \frac{C_{1}(\varepsilon)}{C_{2}(\varepsilon)},
$$

where $\xi_{\mathrm{g}}$ is the geometrical factor and $\xi_{\mathrm{m}}$ is the material factor of the overall normalized resistance ratio $\xi$. The strain effect $C_{1,2}(\varepsilon)$ can be further approximated as follows

$$
C_{1,2}(\varepsilon) \approx 1+g_{1,2} \varepsilon_{\mathrm{e}}+G_{1,2} \varepsilon_{\mathrm{p}}+m_{1,2} \varepsilon_{\mathrm{e}}+M_{1,2} \varepsilon_{\mathrm{p}} .
$$


Here, $g_{1,2}$ and $G_{1,2}$ are linearized gauge factors that account for purely geometrical changes caused by elastic and plastic strains, respectively. In contrast, $m_{1,2}$ and $M_{1,2}$ are linearized gauge factors that account for purely material changes, namely reversible and irreversible anisotropic resistivity changes in the principal directions. Then, for small values of elastic and plastic strains, $\xi$ can be also approximated as follows

$$
\xi \approx 1+g \varepsilon_{\mathrm{e}}+G \varepsilon_{\mathrm{p}}+m \varepsilon_{\mathrm{e}}+M \varepsilon_{\mathrm{p}}
$$

where $g=g_{1}-g_{2}$ and $G=G_{1}-G_{2}$ are differences between the respective geometrical gauge factors in the axial and lateral directions, and $m=m_{1}-m_{2}$ and $M=M_{1}-M_{2}$ denote the relative anisotropic texture of resistivity caused by elastic and plastic strain, respectively. In this way, the geometrical $\xi_{\mathrm{g}}$ and material $\xi_{\mathrm{m}}$ factors of the normalized resistance ratio can be expanded into

$$
\xi_{\mathrm{g}} \approx 1+g \varepsilon_{\mathrm{e}}+G \varepsilon_{\mathrm{p}}
$$

and

$$
\xi_{\mathrm{m}} \approx 1+m \varepsilon_{\mathrm{e}}+M \varepsilon_{\mathrm{p}} \approx 1+D A .
$$

Here, $A$ is a measure of the induced electric anisotropy defined as

$$
A=\frac{\rho_{1}-\rho_{2}}{\left(\rho_{1}+\rho_{2}\right) / 2}
$$

where $\rho_{1}$ and $\rho_{2}$ are the principal resistivity values in the $x_{1}$ and $x_{2}$ directions, and $D \approx\left(\xi_{\mathrm{m}}-1\right) / A$ is the directionality factor of the sensor. The sign of $A$ was chosen so that its expectation value is positive for uniaxial stress along the $x_{1}$ axis.

Let us consider a simple strain gauge whose resistivity $R(\varepsilon) \approx R_{0}(1+F \varepsilon)$, where $R_{0}$ is the gauge's unstrained resistance and $F$ is the so-called gauge factor that is defined as its relative sensitivity to the applied strain

$$
F=\frac{\Delta R / R_{0}}{\varepsilon}
$$

where $\Delta R=R-R_{0}$ is the strain-induced resistance change. The sensitivity of the strain gauge is partly due to geometrical effects and partly due to material effects

$$
F=F_{\mathrm{g}}+F_{\mathrm{m}} \text {. }
$$

The geometrical part of the gauge factor is

$$
F_{\mathrm{g}}=1+2 \mathrm{v}
$$

where $v$ is Poisson's ratio. The material part of the gauge factor is

$$
F_{\mathrm{m}}=\frac{\Delta \rho / \rho_{0}}{\varepsilon}
$$

where $\rho_{0}$ is the resistivity of the unstrained material. For most conventional strain gauges, $F_{\mathrm{g}} \approx$ $1.6(v \approx 0.3)$ and $F_{\mathrm{m}} \approx 0.4$ to 0.6 for an overall gauge factor $F \approx 2.0-2.2$. In the following 
sections, we are going to analyze the relative sensitivity of the square-electrode PD sensor to geometrical and material effects following this concept using asymptotic analytical solutions for very thick and very thin specimens [25-27].

\subsection{Geometrical gauge factor}

In an isotropic half-space the spread of electric current from a point source is spherical

$$
E(r)=\rho J(r)=\frac{I \rho}{2 \pi r^{2}},
$$

where $E$ is the radial electrical field, $\rho$ is the resistivity of the material, $J$ is the current density, $I$ is the injected current, and $r$ is the distance from the source. The electric potential can be evaluated as

$$
\varphi(r)=\int_{r}^{\infty} E(r) d r=\frac{I \rho}{2 \pi} \int_{r}^{\infty} \frac{d r}{r^{2}}=\frac{I \rho}{2 \pi r}+\varphi_{0},
$$

where $\varphi_{0}$ is an arbitrary integration constant that corresponds to the assumed electric potential very far away from the injection point. The transfer resistance can be determined by superposition which yields

$$
\frac{\Delta V}{I}=\frac{\rho}{2 \pi}\left(\frac{1}{r_{11}}-\frac{1}{r_{12}}-\frac{1}{r_{21}}+\frac{1}{r_{22}}\right),
$$

where $r_{i j}(i, j=1$ or 2$)$ is the distance between the $i^{\text {th }}$ injection electrode and the $j^{\text {th }}$ sensing electrode. For the sake of simplicity, let us consider only an ideal square-electrode sensor with an initial characteristic dimension $a$ as shown in Figure 2.

After deformation, the characteristic dimensions in the $x_{1}$ and $x_{2}$ directions will be $a_{1}$ and $a_{2}$, respectively. For the measurement in the axial direction, $r_{11}=r_{22}=a_{2}$ and $r_{12}=r_{21}=\sqrt{a_{1}^{2}+a_{2}^{2}}$. Let the current $I^{+}$be injected at $\mathrm{A}$ and $I^{-}$at $\mathrm{B}$, and the voltage $V^{+}$be measured at $\mathrm{D}$ and $V^{-}$at $\mathrm{C}$. Then, the transfer resistances can be determined from the measured voltage differences as follows

$$
R_{1,2}=\frac{\rho}{\pi}\left(\frac{1}{a_{2,1}}-\frac{1}{\sqrt{a_{1}^{2}+a_{2}^{2}}}\right) .
$$

After some algebra, the geometrical part of the sought resistance ratio $\xi=R_{1} / R_{2}$ is

$$
\xi_{\mathrm{g}}=\frac{\sqrt{1+\gamma^{2}}-1}{\sqrt{1+\gamma^{-2}}-1}
$$

where $\gamma=a_{1} / a_{2}$ is the geometrical aspect ratio of the deformed probe. In the case of uniaxial tension in the $x_{1}$ direction, $a_{1}=a(1+\varepsilon)$ and $a_{2}=a(1-v \varepsilon)$. Assuming small strains $\varepsilon<<1$ only, $\gamma=1+(1+v) \varepsilon$ and the geometrical factor of the resistance ratio $\xi_{\mathrm{g}}$ can be approximated as follows 


$$
\xi_{\mathrm{g}} \approx 1+n(1+v) \varepsilon
$$

where $n=\sqrt{2} /(\sqrt{2}-1) \approx 3.41$. It should be mentioned that although elastic strains are inherently small, plastic strains that occur in later stages of creep could be as high as $50 \%$ depending on the material and the creep conditions therefore the above described simple linearization breaks down as rupture approaches.

Similar calculations can be conducted for a thin plate as well. In an isotropic thin plate the spread of electric current from a point source is cylindrical, therefore

$$
E(r)=\rho J(r)=\frac{I \rho}{2 \pi r t},
$$

where $t$ is the thickness of the plate. The electric potential distribution can be evaluated as

$$
\varphi(r)=\int_{r}^{\infty} E(r) d r=\frac{I \rho}{2 \pi t} \int_{r}^{\infty} \frac{d r}{r}=-\frac{I \rho}{2 \pi t} \ln r+\varphi_{0}
$$

and the transfer resistance can be again determined by superposition which yields

$$
\frac{\Delta V}{I}=-\frac{\rho}{2 \pi t}\left[\ln r_{11}-\ln r_{12}-\ln r_{21}+\ln r_{22}\right] \text {. }
$$

After calculating the voltage difference in a similar fashion as in the case of a half-space, the transfer resistances in the axial $x_{1}$ and lateral $x_{2}$ directions can be obtained as follows

$$
R_{1,2}=\frac{\rho}{2 \pi t} \ln \frac{a_{1}^{2}+a_{2}^{2}}{a_{2,1}^{2}} .
$$

Thus, the geometrical part of the resistance ratio is

$$
\xi_{\mathrm{g}}=\frac{\ln \left(1+\gamma^{2}\right)}{\ln \left(1+\gamma^{-2}\right)}
$$

which can be approximated for small strains exactly as before for a half-space in Eq. (20) with the exception that for thin plates the coefficient $n=2 / \ln (2) \approx 2.88$ is somewhat smaller.

The half-space and thin-plate approximations can be used for specimens of finite thickness whenever $a<<t$ and $t<<a$, respectively. When the plate thickness $t$ is comparable to the electrode separation $a$, exact solutions can be obtained only in the form of an infinite series. The above calculated two asymptotic values represent upper (3.41) and lower (2.88) bounds for $n$ that ranges between these two values depending on the thickness of the plate relative to the electrode separation. Therefore, for typical values of Poisson's ratio $(v \approx 0.3)$, the elastic geometrical gauge factor $f$ ranges between 3.75 and 4.44. The plastic geometrical gauge factor $F$ is a little higher between 4.33 and 5.12 because in the presence of plastic flow the effective Poisson's ratio is $v \approx 0.5$. In short, the square-electrode PD sensor has a geometrical gauge factor that is more than twice that of conventional strain gauges.

Of course, the gauge function $\xi(\gamma)$ is far from linear for large deformations, which has to be taken into consideration whenever the geometrical aspect ratio $\gamma$ exceeds a value of $\approx 1.1$, which is likely to happen during creep well before rupture. The simplistic concept of gauge factor is based on a linearized approximation of the gauge function that is limited to small deformations 
only. For large deformations, a power approximation of the gauge function $\xi \approx \gamma^{n}$ is more accurate, where $n$ is exactly the same as before since $\gamma^{n} \approx 1+n(\gamma-1)$ for $\gamma \approx 1$. Figure 3 illustrates the linear and power approximations of the gauge function for (a) thick and (b) thin plates based on Eqs. (19) and (25), respectively. In most cases, the maximum creep strain reaches about $30 \%$ just before rupture so that the peak value of $\gamma \approx 1+(1+v) \varepsilon \approx 1.5$ while the normalized resistance ratio might reach $\xi \approx 3-4$. In this range of practical interest, the linear approximation might cause more than $20 \%$ underestimation in the normalized resistance ratio while the power approximation is accurate within $\approx 0.5 \%$.

a)

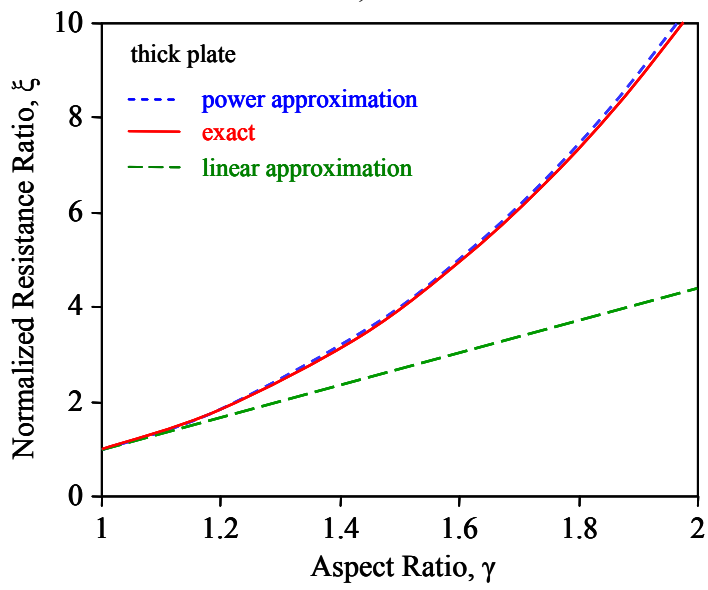

b)

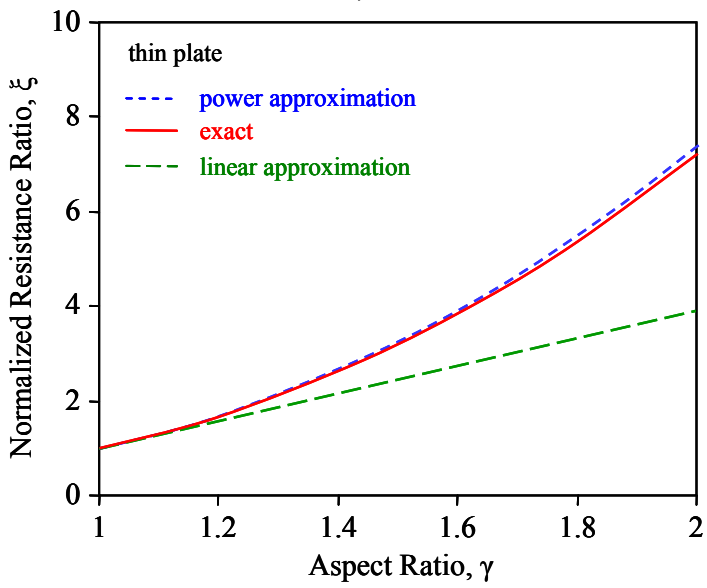

Fig. 3 Linear and power approximations of the gauge function for (a) thick and (b) thin plates.

\subsection{Material gauge factor}

Potential drop measurements with square-electrode configuration are also sensitive to the electric anisotropy of the material, which makes the sensor suitable for detecting variations due to creepinduced texture. In an anisotropic half-space with its free surface lying in the $\left(x_{1}, x_{2}\right)$ principal plane, the potential distribution of a point current source is [28-30]

$$
\varphi=\frac{I \sqrt{\rho_{1} \rho_{2} \rho_{3}}}{2 \pi} \frac{1}{\sqrt{\rho_{1}\left(x_{1}-x_{1 S}\right)^{2}+\rho_{2}\left(x_{2}-x_{2 S}\right)^{2}+\rho_{3}\left(x_{3}-x_{3 S}\right)^{2}}}+\varphi_{0},
$$

where $x_{1}, x_{2}$ and $x_{3}$ are the spatial coordinates of the field point, $x_{1 S}, x_{2 S}$ and $x_{3 S}=0$ are the coordinates of the source point on the surface, and $\rho_{1}, \rho_{2}$ and $\rho_{3}$ denote the electric resistivity in the principal directions. Of course, for sensing points on the surface $x_{3}=0$.

Following the previously used procedure for a square-electrode PD sensor,

$$
R_{1,2}=\frac{\sqrt{\rho_{1} \rho_{2} \rho_{3}}}{\pi a}\left(\frac{1}{\sqrt{\rho_{2,1}}}-\frac{1}{\sqrt{\rho_{1}+\rho_{2}}}\right),
$$

and the material part of the resistance ratio is 


$$
\xi_{\mathrm{m}}=\frac{\sqrt{1+\rho_{1} / \rho_{2}}-1}{\sqrt{1+\rho_{2} / \rho_{1}}-1} .
$$

For the sake of simplicity, let us assume that the initial anisotropy is negligible $\left(\rho_{10} \approx \rho_{20}\right)$. Then, for small values of induced anisotropy $A$ from Eq. (10) the material factor of the normalized resistance ratio $\xi_{\mathrm{m}}$ can be approximated as follows

$$
\xi_{\mathrm{m}} \approx \frac{\frac{2}{\sqrt{2-A}}-1}{\frac{2}{\sqrt{2+A}}-1} \approx 1+\frac{2-\sqrt{2}}{6-4 \sqrt{2}} A
$$

and the directionality factor of the square-electrode sensor defined in Eq. (9) is $D \approx 1.71$.

Similarly, in a thin anisotropic plate and assuming that $x_{1}, x_{2}$ and $x_{3}$ are aligned with the principal axes of conductivity, the potential function has been derived by Tatarnikov [30] as

$$
\varphi=-\frac{I \sqrt{\rho_{1} \rho_{2}}}{2 \pi T} \ln \sqrt{\rho_{1} x_{1}^{2}+\rho_{2} x_{2}^{2}}+\varphi_{0} .
$$

The potential differences and the resulting transfer resistances can be derived like before. After some algebra,

$$
R_{1,2}=\frac{\sqrt{\rho_{1} \rho_{2}}}{2 \pi t} \ln \left(1+\frac{\rho_{1,2}}{\rho_{2,1}}\right)
$$

and the resistance ratio becomes

$$
\xi_{\mathrm{m}}=\frac{\ln \left(1+\frac{\rho_{1}}{\rho_{2}}\right)}{\ln \left(1+\frac{\rho_{2}}{\rho_{1}}\right)} .
$$

Assuming again that the initial anisotropy is negligible and that the induced anisotropy is small

$$
\xi_{\mathrm{m}}=\frac{\ln \left(\frac{4}{2-A}\right)}{\ln \left(\frac{4}{2+A}\right)} \approx 1+\frac{A}{\ln (2)},
$$

therefore the directionality factor of the square-electrode sensor is $D \approx 1.44$.

In summary, the square-electrode PD sensor's sensitivity to anisotropy $D$ ranges between 1.44 and 1.71 depending on the thickness of the specimen relative to the electrode separation. It is interesting to mention that the directionality factor of a conventional in-line PD sensor on a half-space is $D=-0.5$, i.e., negative. It is also somewhat counterintuitive that for a thin plate, the directionality factor of a conventional in-line PD sensor is not only small, but exactly zero [30].

The weak creep-induced electric anisotropy $A \approx A_{\mathrm{e}}+A_{\mathrm{p}}$ can be crudely approximated as a sum of elastic $A_{\mathrm{e}}$ and plastic $A_{\mathrm{p}}$ contributions produced by reversible and irreversible piezoresistivity, respectively. Both effects are relatively weak, therefore, for the sake of 
simplicity, an essentially linear relationship between induced anisotropy and strain can be assumed, so that $A_{\mathrm{e}} \approx p \varepsilon_{\mathrm{e}}$ and $A_{\mathrm{p}} \approx P \varepsilon_{\mathrm{p}}$, where $p$ and $P$ are linearized coefficients of elastic and plastic piezoresistivity, respectively. However, in some cases the nonlinearity of the plastic contribution is so pronounced that it is better to refer to $A_{\mathrm{p}}\left(\varepsilon_{\mathrm{p}}\right)$ in terms of a general function.

Piezoresistivity is defined as the stress dependence of the electric resistivity in the material. In the presence of elastic stress $\tau$, the electric resistivity $[\rho]$ tensor of an initially isotropic conductor becomes slightly anisotropic. In a normalized form, the linearized elastic piezoelectric relationship can be written as

$$
\left[\begin{array}{c}
\Delta \rho_{1} / \rho_{0} \\
\Delta \rho_{2} / \rho_{0} \\
\Delta \rho_{3} / \rho_{0}
\end{array}\right] \approx\left[\begin{array}{lll}
p_{11} & p_{12} & p_{12} \\
p_{12} & p_{11} & p_{12} \\
p_{12} & p_{12} & p_{11}
\end{array}\right]\left[\begin{array}{c}
\tau_{1} / E \\
\tau_{2} / E \\
\tau_{3} / E
\end{array}\right]
$$

where $E$ is Young's modulus, $\Delta \rho_{i}=\rho_{i}-\rho_{0}(i=1,2,3)$ and $\rho_{0}$ represents the electric resistivity in the absence of stress. In terms of the corresponding un-normalized piezoresistivity tensor $[\pi]$ of the material, $p_{11}=E \pi_{11}$ and $p_{12}=E \pi_{12}$ are the unitless axial and lateral piezoresistivity coefficients [31]. In general, $p$ is an intrinsic material parameter that has a relatively modest magnitude in the range of $0.5-1.0$.

The relationship between electric resistivity and plastic strain is usually more nonlinear than the corresponding elastic relationship and strongly depends on the microstructure of the material. For weak effects, the linearized plastic piezoresistivity coefficient $P$ can be defined in a conceptually similar manner as the elastic parameter $p$

$$
\left[\begin{array}{c}
\Delta \rho_{1} / \rho_{0} \\
\Delta \rho_{2} / \rho_{0} \\
\Delta \rho_{3} / \rho_{0}
\end{array}\right] \approx\left[\begin{array}{lll}
P_{11} & P_{12} & P_{12} \\
P_{12} & P_{11} & P_{12} \\
P_{12} & P_{12} & P_{11}
\end{array}\right]\left[\begin{array}{c}
\varepsilon_{\mathrm{p} 1} \\
\varepsilon_{\mathrm{p} 2} \\
\varepsilon_{\mathrm{p} 3}
\end{array}\right] .
$$

The magnitude of $P$ is also relatively modest; it is often in the range of $1-2 \%$ for typical creep levels, i.e., well below its linear counterpart $p$. Therefore, overall, this creep monitoring technique is more sensitive to creep-induced dimensional variations than to creep-induced material changes that are sometimes non-monotonic functions of the damage accumulation.

\subsection{Oriented clusters of microcracks}

Most creep-induced crystallographic defects, e.g., dislocations, exert a negligible influence on the electric resistivity of metals at room or elevated temperatures. Increasing precipitate size and number density might significantly change, usually decrease, the electric conductivity in metals, but the change is essentially isotropic, i.e., the same in the principal creep direction and normal to it, therefore will not affect our estimate of the creep strain in the case of uniaxial creep considered here since only the resistance ratio between the axial and transverse directions is used for inversion purposes. We will illustrate later that under biaxial creep, isotropic effects such reversible temperature changes or irreversible microstructural changes cannot be so easily suppressed by evaluating the resistance ratio only, therefore even isotropic resistivity changes will affect our measurements. However, in the simpler case of creep under uniaxial stress considered in this section, only anisotropic resistivity changes can influence the measured results. 
In later stages of creep, oriented clusters of grain boundary cavities form that leads to oriented grain boundary separation usually normal to the principal creep direction. In contrast with other material effects, such oriented discontinuities might lead to significant electric anisotropy. As an example, let us consider an oriented cluster of randomly distributed pennyshaped cracks. For the sake of simplicity, let us also assume that all the cracks have the same diameter of $d$ and they are all normal to the inspection direction. Then the normalized conductivity change can be expressed with the following second-order tensor

$$
\left[\begin{array}{c}
\Delta \rho_{1} / \rho_{0} \\
\Delta \rho_{2} / \rho_{0} \\
\Delta \rho_{3} / \rho_{0}
\end{array}\right] \approx \delta\left[\begin{array}{lll}
1 & 0 & 0 \\
0 & 0 & 0 \\
0 & 0 & 0
\end{array}\right] .
$$

where $\delta$ is the normalized excess resistivity in the inspection direction that can be calculated from the volume density $N$ of the cracks [32] as follows

$$
\delta=\frac{d^{3}}{3} N
$$

On optical micrographs of the polished surface of creep damaged materials, or the replicas of such surfaces, one can count the number of visible cracks and calculate the area number density $n$ of them. All those penny-shaped cracks whose center is within $\pm d / 2$ of the section will be cut and therefore will show up on the surface to be inspected. Their length on the surface will be smaller than their actual diameter depending on how far from their center they were cut by the sectioning plate, therefore one can estimate their diameter based on the largest observed length. Then, the area number density of the cracks on the surface will be $n=N d$ so that

$$
\delta=\frac{d^{2}}{3} n
$$

Assuming that typical crack sizes are around of $d \approx 25 \mu \mathrm{m}$ and the area number density could be on the order of $n \approx 100 \mu \mathrm{m}^{-2}$, the creep cracking induced electric anisotropy is $\delta \approx 0.02$ or $2 \%$.

\subsection{Inversion for creep under uniaxial stress}

Since material changes are expected to be negligible with respect to the accompanying geometrical changes, inversion of the measured electric resistances can be conducted by assuming pure geometrical effect. Such approximations are expected to be acceptable throughout the primary and secondary stages of creep up to the last fraction of tertiary creep when oriented clusters of grain boundary separations and microcracks coalesce into larger macrocracks and ultimately lead to the formation of a terminal crack and rupture. As a result, the inverted NDE strain $\varepsilon_{\mathrm{NDE}}$ is expected to be a good measure of the actual local plastic strain except maybe the last few percents of creep life when it inherently overestimates the true strain.

In the previous section we demonstrated that the measured resistance ratio $\xi$ between the axial and lateral directions is mainly a function of the aspect ratio $\gamma=a_{1} / a_{2}$ of the electrode configuration. Assuming an initially square electrode configuration $\left(a_{10}=a_{20}\right)$, the aspect ratio can be estimated in the small strain approximation as

$$
\gamma=\frac{1+\varepsilon_{1}}{1+\varepsilon_{2}} \approx 1+\left(\varepsilon_{1}-\varepsilon_{2}\right)
$$


Under the additional simplifying conditions of uniaxial stress, the electrode aspect ratio can be further simplified as follows

$$
\gamma \approx 1+\varepsilon_{1}(1+v)
$$

where we used the axial strain $\varepsilon_{1}$ as a single parameter to characterize the creep and $v$ denotes Poisson's coefficient that is assumed to be 0.5 for plastic strains. In early stages of creep degradation we can use the simple linearized concept of geometrical gauge factor $G=n(1+v)$

$$
\xi \approx 1+G \varepsilon_{1}
$$

for inversion purposes so that we get

$$
\varepsilon_{\mathrm{NDE}} \approx \frac{\xi-1}{1.5 n}
$$

where $n=2.88$ for thin plates and $n=3.41$ for thick ones.

As Figure 3 illustrated it, Eq. (41) is valid only for aspect ratios of 1.1 or less, i.e., plastic strains of $2 \%$ or less. However, plastic strains as high as $10-30 \%$ can be expected during creep before rupture, therefore the linearized approximation will cause unnecessary errors (overestimations) in the actual plastic strain. Much better results can be obtained by using the above introduced nonlinear approximation of the resistance ratio

$$
\xi \approx \gamma^{n}
$$

Combining Eqs. (39) and (41) leads to a nonlinear inversion equation as follows

$$
\varepsilon_{\mathrm{NDE}} \approx \frac{\xi^{1 / n}-1}{1+\frac{\xi^{1 / n}}{2}} .
$$

Figure 4 shows a comparison between the linearized inversion formula of Eq. (42) and the improved nonlinear inversion formula of Eq. (44) for a thick plate $(n=3.41)$. The experimental data was obtained in a uniaxial creep test of $9 \mathrm{Cr}-1 \mathrm{Mo}$ specimen at $120 \mathrm{MPa}$ and $650{ }^{\circ} \mathrm{C}$. It is evident, that beyond $50 \%$ of the creep rupture time the linear approximation introduced originally for its conceptual simplicity is not sufficient for accurate quantitative materials characterization purposes. 
a) analytical

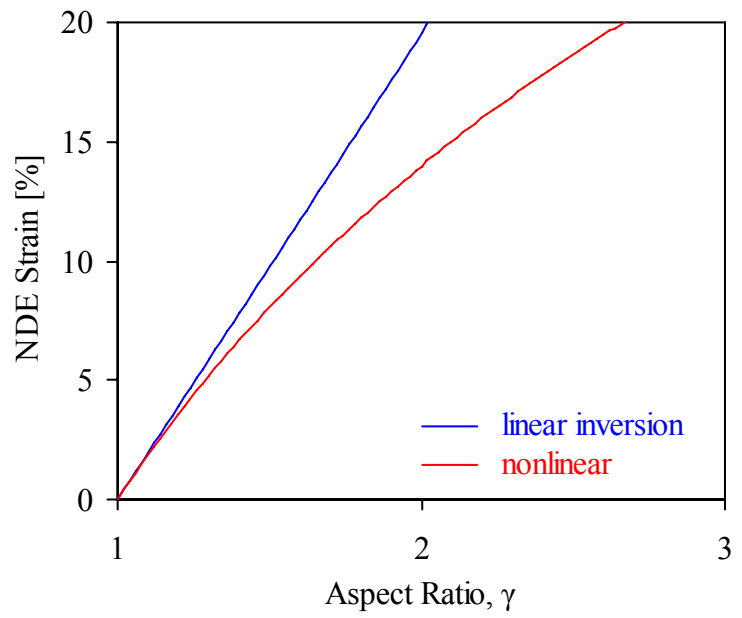

b) experimental

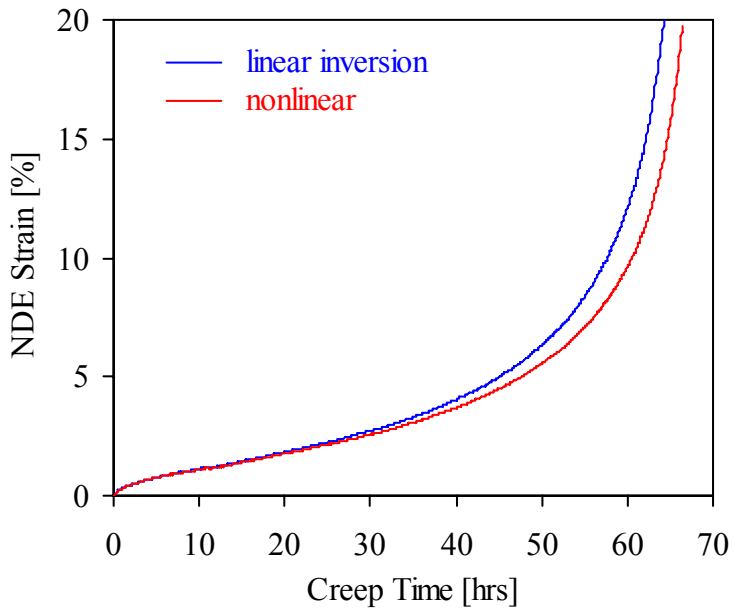

Fig. 4 Comparison between the linearized and nonlinear inversion formulas for a thick plate.

The experimental data was obtained in a uniaxial creep test of $9 \mathrm{Cr}-1 \mathrm{Mo}$ specimen at $120 \mathrm{MPa}$ and $650^{\circ} \mathrm{C}$.

\subsection{Inversion for creep under biaxial stress}

For the purposes of laboratory tests, it is sufficient to consider creep under uniaxial stress, when the axial $\varepsilon_{1}$ and lateral $\varepsilon_{2}$ plastic strains are related to each other via the Poisson effect that assures that the volume of the material remains essentially the same therefore $\varepsilon_{2}=-\varepsilon_{1} / 2$. In the more general case of creep under biaxial stress, the axial $\varepsilon_{1}$ and lateral $\varepsilon_{2}$ plastic strains will depend on the ratio of the axial $\tau_{1}$ and lateral $\tau_{2}$ principal stresses (the third principal stress is normal to the monitored surface therefore vanishes $\tau_{3}=0$ ). For the sake of simplicity, we will limit our discussion of biaxial stress to the case of a thick plate, but similar results can be obtained for thin plates as well. Let us assume that an initially square electrode system $\left(a_{10}=a_{20}\right.$ $=a)$ is mounted on a thick plate. From Eq. (18), the two resistances measured in the $x_{1}$ and $x_{2}$ directions are

$$
R_{1,2}=\frac{\rho}{\pi a}\left(\frac{1}{1+\varepsilon_{2,1}}-\frac{1}{\sqrt{\left(1+\varepsilon_{1}\right)^{2}+\left(1+\varepsilon_{2}\right)^{2}}}\right) .
$$

Assuming that the creep induced material anisotropy is weak, the electric resistivity $\rho$ changes mainly because of reversible temperature variations and, to a much lesser degree, because of irreversible thermally-induced microstructural evolution (e.g., precipitation coarsening). Inversion requires the estimation of the principal plastic strains from the measured resistances. The difficulty lies in the fact that we have three unknowns, namely the two strains $\varepsilon_{1,2}$ and the electric resistivity $\rho$, but only two measurements, namely $R_{1,2}$, therefore only two equations for three unknowns. In the simpler case of uniaxial creep we could exploit that $\varepsilon_{2}=-\varepsilon_{1} / 2$ and suppress the reversible and irreversible temperature variations of $\rho$ by considering the resistivity ratio $R_{1} / R_{2}$. 
In the case of creep under biaxial stress, the ratio between the principal strains is not known a priory. The first step is still normalizing the measured resistances to their initial values

$$
\eta_{1,2}=n \frac{\rho}{\rho_{0}}\left(\frac{1}{1+\varepsilon_{2,1}}-\frac{1}{\sqrt{\left(1+\varepsilon_{1}\right)^{2}+\left(1+\varepsilon_{2}\right)^{2}}}\right),
$$

where $n=\sqrt{2} /(\sqrt{2}-1) \approx 3.41$ is a geometrical factor and $\rho_{0}$ is the initial resistivity of the material at a reference temperature $T_{0}$. Nonlinear inversion based on the power approximation of Eq. (43) leads to

$$
\varepsilon_{1,2} \approx n \frac{\rho}{\rho_{0}} \frac{\left(\frac{\eta_{1,2}}{\eta_{2,1}}\right)^{1 / n}-1}{\eta_{1,2}-\eta_{2,1}}-1,
$$

where the $\rho / \rho_{0}$ ratio is essentially unknown. The reversible temperature variation of the electric resistivity can be fairly easily compensated by either measuring the temperature or measuring the resistance of the material at a location where creep is known to be negligible, but in practice both approaches are fraught with difficulties. The biaxial inversion of Eq. (47) sacrifices the almost perfect thermal common mode rejection ratio (CMRR) of the uniaxial inversion. Let us assume that a small uncorrected isotropic increase $\delta$ occurred in the electric resistivity, i.e., $\Delta \rho / \rho_{0}=\delta$. Then both inverted strains will exhibit an error of $\Delta \varepsilon_{1,2}=-\delta$. This additive error is relatively high compared to typical plastic strain levels that occur during secondary creep, therefore will have to be compensated for in case of field monitoring of creep under biaxial stress conditions.

\section{Directional Potential Drop Measurement for Manual Creep Characterization}

Detectability of creep damage in its early stages is of major importance because cracks may develop and propagate very rapidly as rupture approaches. These cracks are most likely to originate in the critical areas of pre-existing defects or stress concentrations. The most critical locations tend to be in the vicinity of welds. Depending on their location, creep cracks have been classified into four types [2]. Type I cracks develop in the weld metal. Type II cracks form in the weld metal and propagate towards the coarse grains in the heat-affected zone (HAZ). Type III cracks originate in the coarse-grain region of the HAZ, whereas Type IV cracks develop in the transition region between the fine-grain region of the HAZ and the base metal, where the creep strength is typically lower. This transition region between the HAZ and the base metal tends to have the highest concentration of diffusion cavities and is the most likely area for creep crack initiation due to hoop, residual or bending stresses [33-38]. Monitoring creep damage accumulation in its early stage has been a major concern in the NDE community for quite some time. From numerous NDE methods, potential drop measurement emerged as one of the most promising options [2]. Alternating current potential drop (ACPD) measurement is an electromagnetic technique used mainly for monitoring crack growth [39,40], estimating the depth of surface breaking defects $[41,42]$ and evaluating material properties such as conductivity or permeability $[43,44]$. The main advantage of ACPD mode of operation over DCPD is that the same injection current produces much larger potential differences [45]. Using this technique the measured resistance increases when the probe overlaps a defect because the injected current is forced to travel around and underneath the defect, such as a surface breaking crack, which leads to increasing potential drop between the sensing electrodes $[46,47]$. 
In this project we also investigated the feasibility of low-frequency directional ACPD (DACPD) evaluation of creep damage close to the boundary separating a weld from the base metal of typically significantly higher electric conductivity. In the previous section, only permanently installed probes were considered that are mainly sensitive to the purely geometrical effects of creep due to plastic strain. In this section we focus on deployable probes that are sensitive to the material effects of creep only. We will present analytical and computational results to assess the feasibility of DACPD with deployable electrodes to quantitatively assess the apparent local electric anisotropy produced by the interface between regions of different electric conductivity, which ultimately limits the detection threshold of creep damage in the vicinity of welds.

\subsection{Deployable DACPD probe}

A spring-loaded deployable DACPD probe can be used to measure the electrical anisotropy of conductors without the previously described geometrical effect that dominates measurements using permanently attached electrodes. Figure 5 shows an image of the deployable squareelectrode DACPD probe. It consists of four spring-loaded heavy-duty pins with $a=4 \mathrm{~mm}$ separation that are embedded in a Plexiglas fixture. The technique uses low-frequency ACPD to measure two resistances in two orthogonal directions at the same location as it was shown in Figure 2. Since the electrodes are sprung-loaded, the average electrode separation remains constant independent of the plastic deformation the material might have undergone between measurements, though slight random variations are inevitable due to the limited targeting accuracy of deployable electrodes. Because of this, the probe is not sensitive to geometrical effects of creep strain and can be used to detect selectively material changes only.

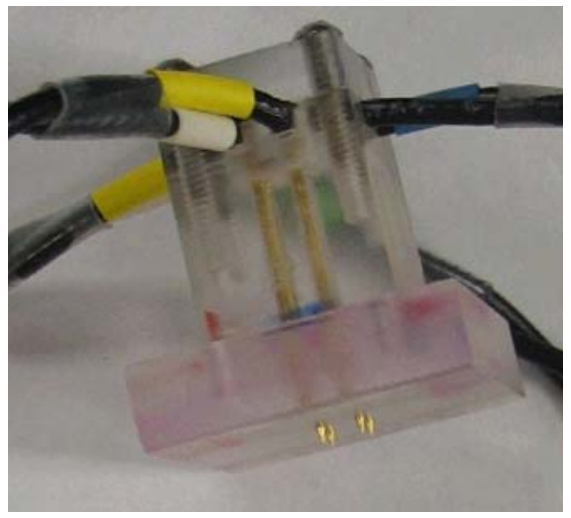

Fig. 5 A picture of a deployable DACPD probe with spring-loaded electrode pins.

The best commercially available spring loaded electrodes exhibit $\pm 50 \mu \mathrm{m}$ targeting accuracy which, unless further reduced, could cause as much as $\Delta a / a \approx \pm 2 \%$ relative electrode separation uncertainty. Since the geometrical gauge factor of the DACPD probe is around $G \approx 5$, such positioning uncertainty causes as much as $\pm 10 \%$ random variation in the sought $R_{1} / R_{2}$ resistance ratio, which is unacceptable in most measurements. Therefore, we further reduced the electrode positioning uncertainty by the adding a Plexiglas guiding plate to the front of the probe.

In order to illustrate the directional sensitivity of the DACPD probe, electrical anisotropy measurements were conducted on an isotropic stainless steel 304 plate and on an anisotropic textured Ti-6Al-4V plate. The results of these DACPD resistance measurements are shown in Figures 6(a) and 6(b), respectively. One hundred readings were taken at the center of the plates 
at two orthogonal probe orientations alternating between axial $\left(0^{\circ}\right)$ and lateral $\left(90^{\circ}\right)$ directions. Rotation of the probe was necessary to suppress inevitable small differences between the electrode separations in the two measurement directions. Each resistance data point is the average of the two readings, i.e., the $0^{\circ}$-measurement taken at axial probe orientation was averaged with the $90^{\circ}$-measurement taken at lateral probe orientation. Similarly, the $90^{\circ}$ measurement taken at axial probe orientation was averaged with the $0^{\circ}$-measurement taken at lateral probe orientation. The two averaged values of the electric resistance were then normalized to the combined average resistance.

It is well known that cubic materials do not exhibit crystallographic anisotropy in their electric properties, while those preferentially crystallizing in hexagonal symmetry, like Titanium alloys, do [48]. The normalized resistance results obtained on textured Ti-6Al-4V illustrate that the DACPD probe can easily detect the approximately $4 \%$ electric conductivity anisotropy caused by crystallographic texture, while the isotropic 304 stainless steel plate does not exhibit any perceivable anisotropy. The scattering of the data at each orientation is less than $1 \%$ and mainly due to the uncertainty in pin positioning that increases the random error of the measurement well above the intrinsic electric noise. Based on these results, this DACPD probe could be used to evaluate creep-induced electric anisotropy above about $1 \%$, so it is expected to be sensitive to material effects of creep in its advanced stages of damage accumulation.

a)

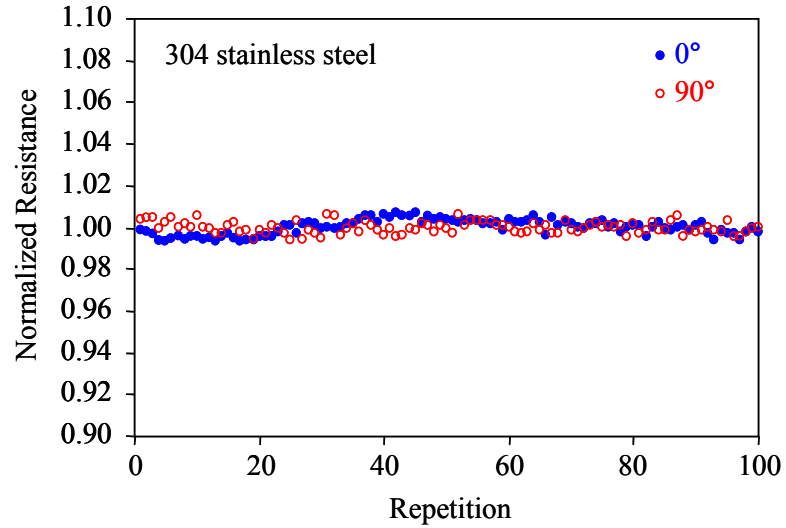

b)

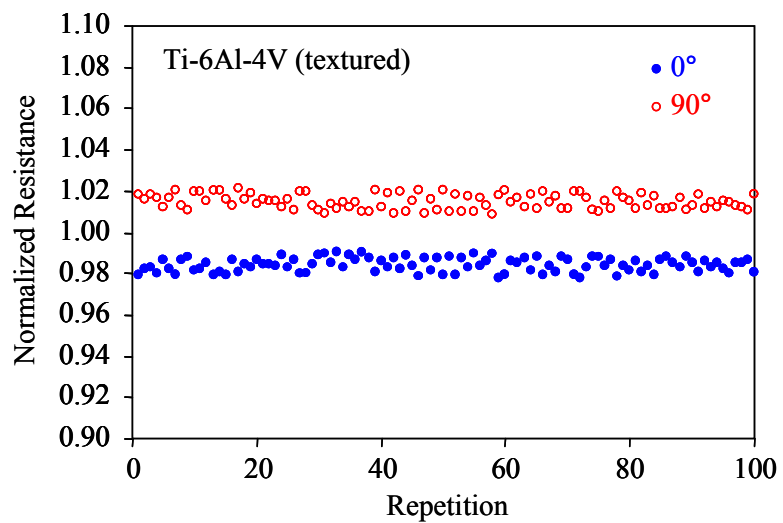

Fig. 6 DACPD resistance measurements in (a) 304 stainless steel and (b) textured Ti-6Al-4V plates.

These results indicate that the deployable DACPD probe is capable of detecting and quantitatively assessing electric resistivity anisotropy on the surface of conducting components. However, it is also sensitive to directional conductivity variations in otherwise isotropic materials. For example, it registers an apparent anisotropy in the vicinity of the boundary between two conductive domains of different resistivity even when both domains are individually isotropic. In the next session we will investigate the influence of this apparent anisotropy on creep monitoring in the vicinity of weld/base metal interfaces.

\subsection{Apparent anisotropy of a normal boundary between two conducting domains}

First, we analyzed the simplest case of a normal plane boundary separating two conducting halfspaces. The Method of Images was used to derive the resistance equations at two orthogonal orientations either normal or tangential to the boundary [49]. The radial component of the spherical potential distribution produced by a single current source $I$ on the surface of a 
homogeneous isotropic half-space can be calculated from Gauss' law as it was given in Eq. (16). Figure 7(a) shows the geometrical configuration when the source point $\mathrm{A}\left(x_{\mathrm{s}}, y_{\mathrm{s}}\right)$ and the sensing point $\mathrm{B}(x, y)$ are on the same side of the interface separating two quarter-spaces of electric conductivity $\sigma_{1}$ and $\sigma_{2}$. According to the Method of Images, the electric field on the injection side of the boundary can be calculated by superposition of the direct field produced by the injection current $I$ at $\mathrm{A}\left(x_{\mathrm{s}}, y_{\mathrm{s}}\right)$ and the reflected image of the injection source $I$ ' placed at $\mathrm{A}^{\prime}\left(x_{\mathrm{s}}\right.$, $y_{\mathrm{s}}$ ) while assuming that the medium is homogeneous with an electric conductivity equal to $\sigma_{1}$ everywhere.

Figure $7(\mathrm{~b})$ shows the corresponding geometrical configuration when the source point $\mathrm{A}\left(x_{\mathrm{s}}\right.$, $\left.y_{\mathrm{s}}\right)$ and the sensing point $\mathrm{B}(x, y)$ are on the opposite sides of the interface. Then, the electric field on the sensing side of the boundary can be calculated as the transmitted image of the injection source $I$ " placed at $\mathrm{A}\left(x_{\mathrm{s}}, y_{\mathrm{s}}\right)$ while assuming that the medium is homogeneous with an electric conductivity equal to $\sigma_{2}$ everywhere. The physical boundary conditions at the interface, i.e., the continuity of the normal current density and the tangential electric field, can then be satisfied by the appropriate choice of the $I ' / I$ reflection and $I$ ''/I transmission coefficients as follows [49]

a)
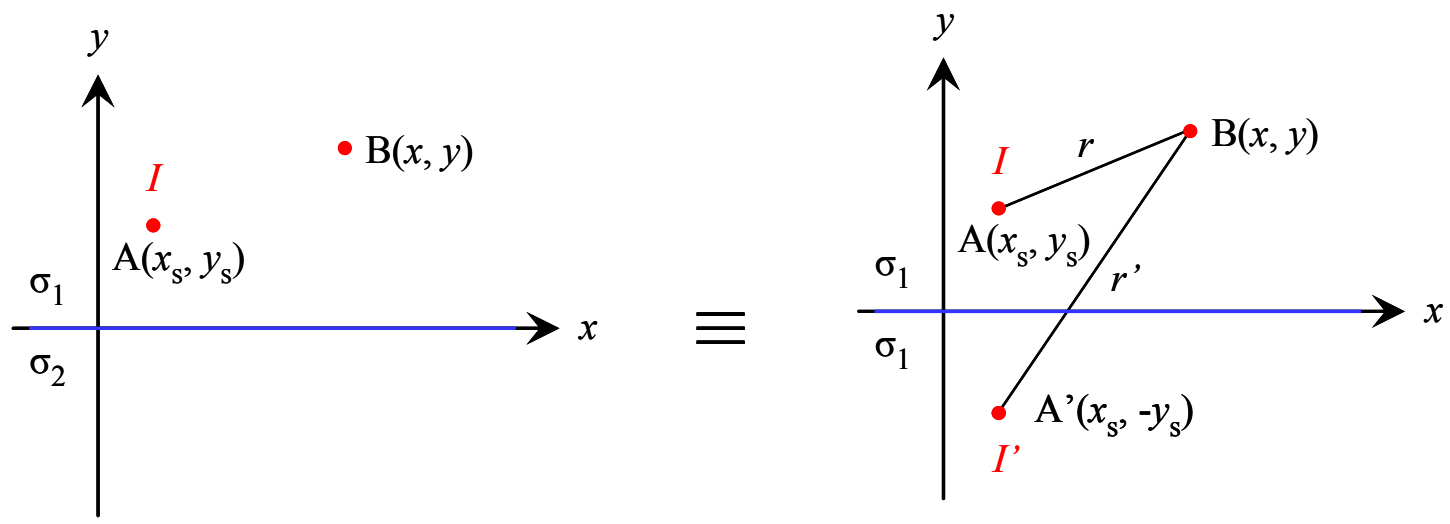

b)
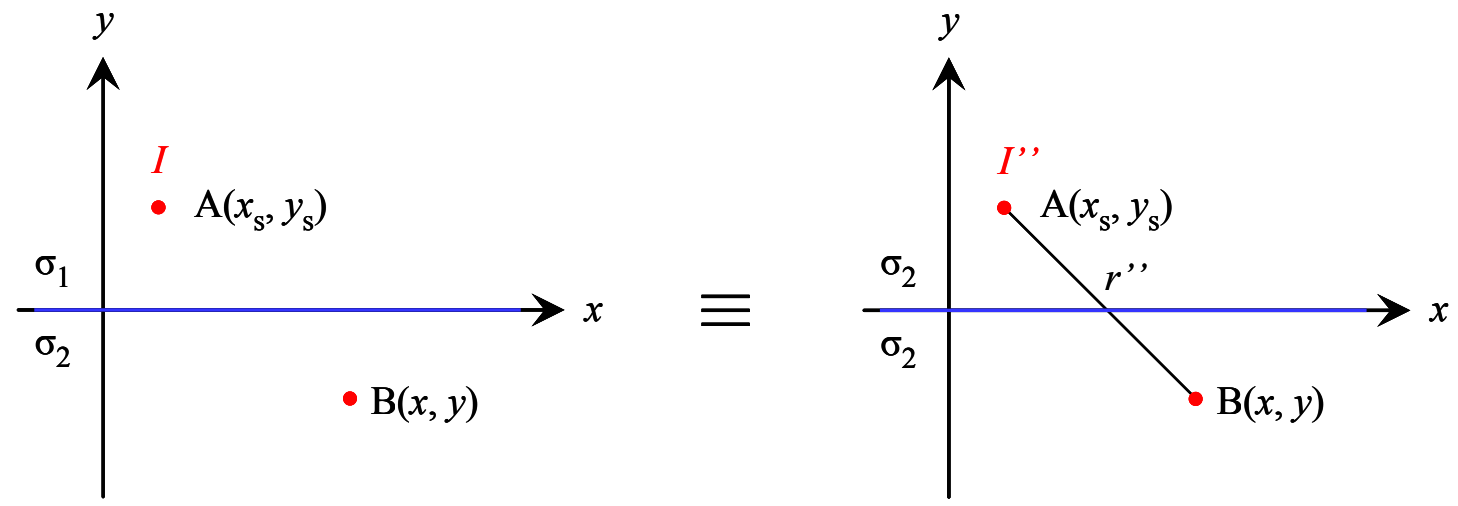

Fig. 7 Illustration of the Method of Images with the injection and sensing points on the same side (a) and opposite sides (b) of the plane boundary separating two conducting quarter-spaces. 


$$
\frac{I^{\prime}}{I}=\frac{\sigma_{1}-\sigma_{2}}{\sigma_{1}+\sigma_{2}}
$$

and

$$
\frac{I^{\prime \prime}}{I}=\frac{2 \sigma_{2}}{\sigma_{1}+\sigma_{2}}
$$

When the injection and sensing points are located on the first side $\left(\sigma_{1}\right)$ of the plane boundary separating two conducting quarter-spaces, the electric potential can be written as

$$
V^{(11)}=\frac{I}{2 \pi \sigma_{1} r}+\frac{I^{\prime}}{2 \pi \sigma_{1} r^{\prime}}=\frac{I}{2 \pi \sigma_{1} \sqrt{\left(x-x_{s}\right)^{2}+\left(y-y_{s}\right)^{2}}}+\frac{I^{\prime}}{2 \pi \sigma_{1} \sqrt{\left(x-x_{s}\right)^{2}+\left(y+y_{s}\right)^{2}}} .
$$

On the other hand, when the injection point is on the first side and the sensing point is on the other side of the plane boundary, the electric potential is

$$
V^{(12)}=\frac{I^{\prime \prime}}{2 \pi \sigma_{2} r^{\prime \prime}}=\frac{I^{\prime \prime}}{2 \pi \sigma_{2} \sqrt{\left(x-x_{s}\right)^{2}+\left(y-y_{s}\right)^{2}}} .
$$

Substituting $I$ ' and $I$ ' from Eqs. (48) and (49) into Eqs. (50) and (51), respectively, the potential distribution can be written as

$$
V^{(11)}=\frac{I}{2 \pi \sigma_{1}}\left[\frac{1}{\sqrt{\left(x-x_{s}\right)^{2}+\left(y-y_{s}\right)^{2}}}+\frac{\sigma_{1}-\sigma_{2}}{\sigma_{1}+\sigma_{2}} \frac{1}{\sqrt{\left(x-x_{s}\right)^{2}+\left(y+y_{s}\right)^{2}}}\right],
$$

when the injection and sensing points are on the same side of the plane boundary and

$$
V^{(12)}=\frac{I}{\pi\left(\sigma_{1}+\sigma_{2}\right)} \frac{1}{\sqrt{\left(x-x_{s}\right)^{2}+\left(y-y_{s}\right)^{2}}},
$$

when the injection and sensing points are on the opposite sides of the plane boundary. When the injection and sensing points are both located on the second side $\left(\sigma_{2}\right)$ of the boundary, the potential $V^{(22)}$ can be obtained from Eq. (52) by simply replacing $\sigma_{1}$ with $\sigma_{2}$ and vice versa. Of course, according to the Law of Reciprocity, the injection and sensing electrodes are interchangeable, therefore $V^{(21)}=V^{(12)}$, as one can easily see from the symmetric form of Eq. (53).

In the case of parallel and normal potential drop measurements across the boundary, the injection and sensing points might be on either the same side or the opposite sides of the boundary separating the two conducting quarter-spaces as shown in Figure 8. Generally, the measured transfer resistance can be calculated from

$$
R=\frac{V^{(+)}-V^{(-)}}{I}
$$

where $I^{(+)}=-I^{(-)}=I$. Using the combination of potential distribution equations obtained above for $V^{(11)}, V^{(22)}, V^{(12)}$ and $V^{(21)}$, the parallel and normal resistances can be readily derived. 
For a probe entirely on the first side $\left(\sigma_{1}\right)$ of the boundary as shown in Figures 8(a) and 8(c), the parallel and normal resistances are

$$
R_{\mathrm{p}}^{(11)}=\frac{1}{\pi \sigma_{1} a}\left[\left(1-\frac{1}{\sqrt{2}}\right)+\frac{\sigma_{1}-\sigma_{2}}{\sigma_{1}+\sigma_{2}}\left(\frac{1}{2 h / a}-\frac{1}{\sqrt{1+(2 h / a)^{2}}}\right)\right]
$$

and

$$
\begin{aligned}
R_{\mathrm{n}}^{(11)}= & \frac{1}{2 \pi \sigma_{1} a} \\
& {\left[2\left(1-\frac{1}{\sqrt{2}}\right)+\frac{\sigma_{1}-\sigma_{2}}{\sigma_{1}+\sigma_{2}}\left(\frac{1}{\sqrt{1+(1+2 h / a)^{2}}}+\frac{1}{\sqrt{1+(1-2 h / a)^{2}}}-\frac{2}{\sqrt{1+(2 h / a)^{2}}}\right)\right], }
\end{aligned}
$$

a)

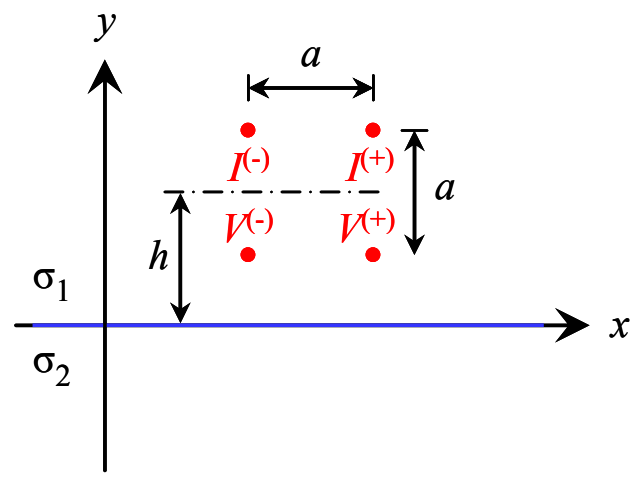

c)

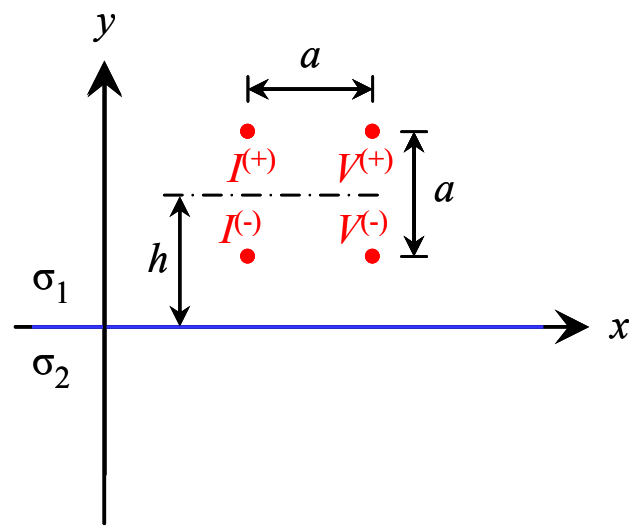

b)

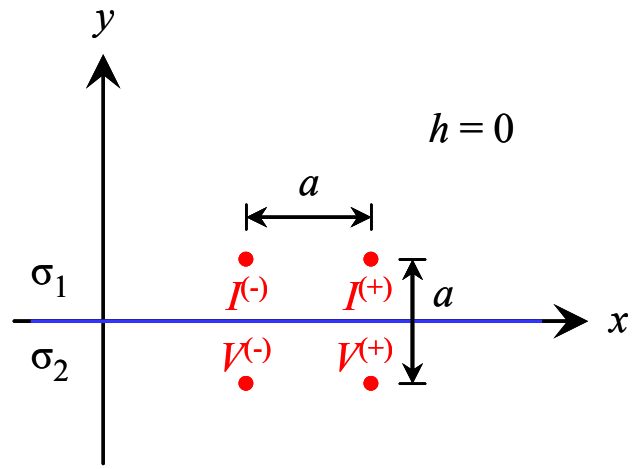

d)

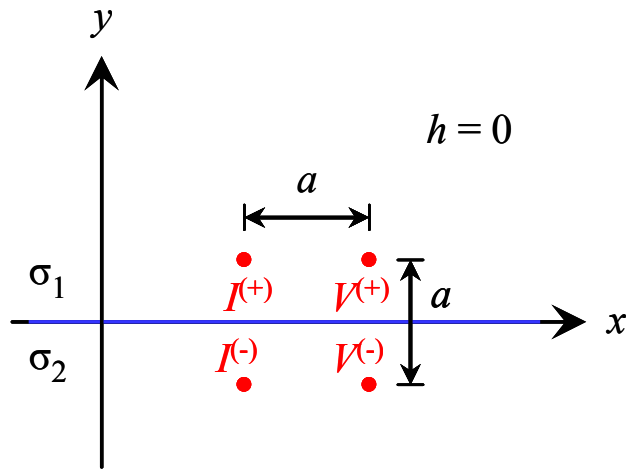

Fig. 8 Parallel $(\mathrm{a}, \mathrm{b})$ and normal $(\mathrm{c}, \mathrm{d})$ potential drop measurements with square-electrode configuration on the same side of the boundary $(a, c)$ and across it $(b, d)$.

where $h$ is the distance from the centerline of the square-electrode probe to the boundary separating the two conducting quarter-spaces. For a probe straddling the boundary as shown in Figures 8(b) and 8(d), the parallel and normal resistances are 


$$
R_{\mathrm{p}}^{(12)}=\frac{2}{\pi\left(\sigma_{1}+\sigma_{2}\right) a}\left(1-\frac{1}{\sqrt{2}}\right)
$$

and

$$
\begin{aligned}
R_{\mathrm{n}}^{(12)}= & \frac{1}{2 \pi\left(\sigma_{1}+\sigma_{2}\right) a} \\
& {\left[4-\frac{4}{\sqrt{2}}-\left(\sigma_{1}-\sigma_{2}\right)\left\{\frac{1}{\sigma_{1}}\left(1-\frac{1}{\sqrt{1+(1+2 h / a)^{2}}}\right)-\frac{1}{\sigma_{2}}\left(1-\frac{1}{\sqrt{1+(1-2 h / a)^{2}}}\right)\right\}\right] }
\end{aligned}
$$

Finally, when the probe is entirely on the second side $\left(\sigma_{2}\right)$ of the boundary, the parallel and normal resistances are

$$
R_{\mathrm{p}}^{(22)}=\frac{1}{\pi \sigma_{2} a}\left[\left(1-\frac{1}{\sqrt{2}}\right)+\frac{\sigma_{2}-\sigma_{1}}{\sigma_{2}+\sigma_{1}}\left(-\frac{1}{2 h / a}-\frac{1}{\sqrt{1+(2 h / a)^{2}}}\right)\right]
$$

and

$$
\begin{aligned}
R_{\mathrm{n}}^{(22)}= & \frac{1}{2 \pi \sigma_{2} a} \\
& {\left[2\left(1-\frac{1}{\sqrt{2}}\right)+\frac{\sigma_{2}-\sigma_{1}}{\sigma_{2}+\sigma_{1}}\left(\frac{1}{\sqrt{1+(1+2 h / a)^{2}}}+\frac{1}{\sqrt{1+(1-2 h / a)^{2}}}-\frac{2}{\sqrt{1+(2 h / a)^{2}}}\right)\right], }
\end{aligned}
$$

where besides replacing $\sigma_{1}$ with $\sigma_{2}$ and vice versa the sign of the probe offset $h$ relative to the interface also had to be inverted, which affects Eq. (59) but not (60).

Using the parallel and normal resistances derived in Eqs. (55) through (60), the normalized average resistance

$$
R_{\mathrm{a}}=\frac{R_{\mathrm{p}}+R_{\mathrm{n}}}{2 R_{\mathrm{r}}}
$$

and normalized anisotropy

$$
A=\frac{R_{\mathrm{p}}-R_{\mathrm{n}}}{2 R_{\mathrm{r}}}
$$

were obtained. These quantities are presented in a dimensionless form normalized with respect to the reference resistance that the same square-electrode probe would measure on a hypothetical homogeneous and isotropic half-space with an electric conductivity equal to the average of the conductivities of the two contacting quarter-spaces. For an ideal square-electrode probe, this reference resistance can be calculated as follows

$$
R_{\mathrm{r}}=\frac{2}{\pi\left(\sigma_{1}+\sigma_{2}\right) a}\left(1-\frac{1}{\sqrt{2}}\right) .
$$


This simplified model can be applied to a weld/base metal interface by assuming that $\sigma_{1}$ is the isotropic conductivity of the base metal, $\sigma_{2}$ is the lower conductivity of the also isotropic weld, and the plane boundary between the quarter-spaces represents the idealized fusion surface between them. Figure 9(a) shows how the normalized average resistance $R_{\mathrm{a}}$ gradually increases from the base metal, through the interface (fusion line), to the weld region for different $\sigma_{1} / \sigma_{2}$ conductivity ratios over a transition region that extends roughly one probe dimension on both sides of the bondline. The normalized apparent anisotropy $A$ exhibits significant values and sharp variations only over the transition region as shown in Figure 9(b) and higher conductivity ratios produce higher apparent anisotropy in the vicinity of the interface. This anisotropy is called "apparent" because the intrinsic electric conductivity is isotropic everywhere and the observed macroscopic anisotropy is caused entirely by the presence of the interface. For 50\% difference between the more conducting base metal and the less conductive weld, the apparent anisotropy could reach as much as $+/-20 \%$ in the worst case scenario which occurs when the probe offset $(h)$ relative to the bondline is such that two pins are directly over the bondline $(h / a=$ $+/-0.5)$. This apparent anisotropy changes so rapidly with offset from the bondline that small positioning errors will inevitably lead to large measurement uncertainties in terms of anisotropy, which explains the large experimental uncertainties observed in our measurements to be introduced later when the probe is repeatedly re-positioned over the bond line.

a)

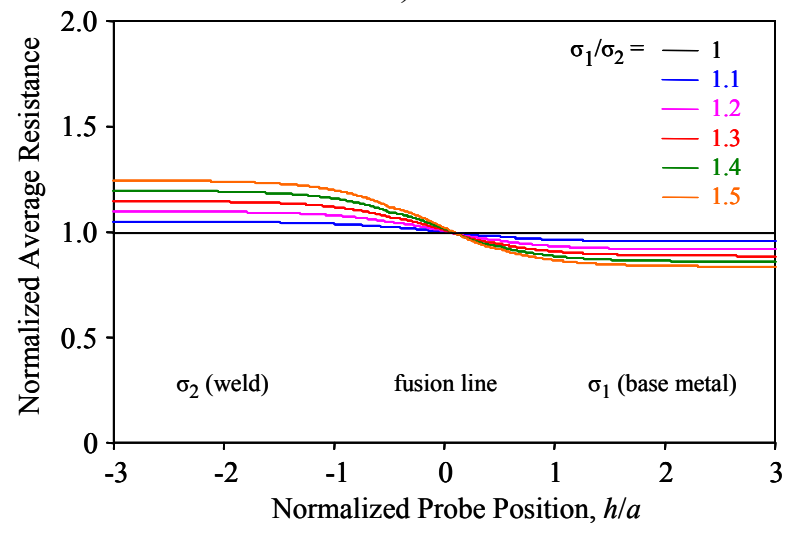

b)

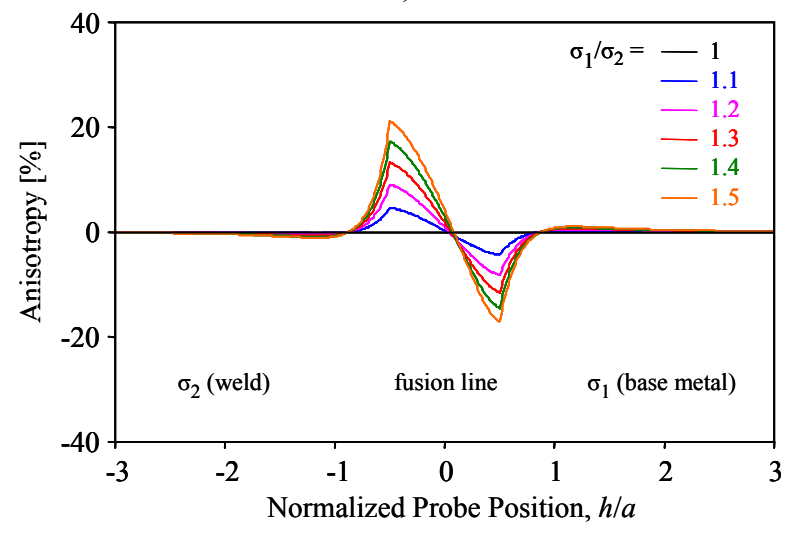

Fig. 9 The normalized average resistance (a) and anisotropy (b) across the three regions (base metal, interface and the weld) as a function of the normalized probe position.

\subsection{Numerical validation of the analytical predictions}

The analytical predictions derived in the previous section showed a gradual increase in the average resistance across the interface from the base metal to the weld region as well as a spurious apparent anisotropy near the bondline (see Figure 9). Although the predicted apparent anisotropy essentially vanishes directly over the bondline, a small offset in either direction could cause a significant variation in the measured anisotropy. This high sensitivity to probe position suggests that there will be an inherently high experimental measurement uncertainty in the vicinity of the weld/base metal boundary. A more quantitative validation of the predicted apparent anisotropy will require a comparison to the results of measurements at various distances from the bondline. Such measurements will be presented later and, with the exception of the most damaged one, will reveal anisotropy profiles $A(h)$ similar to those predicted by the 
simplified analytical model which assumed a perfect normal interface between two connected quarter-spaces of different electric conductivities.

These effects can be much more easily studied by numerical simulation than by more complex analytical models, therefore we used a commercially available finite element analysis software (ANSYS) to numerically validate the analytical predictions for the one case where analytical results were already available, i.e., in the case of two quarter-spaces in perfect electric contact. A three-dimensional geometry was defined that included three regions, the base metal, the interface, and the weld, with a conductivity ratio of $\sigma_{1} / \sigma_{2}=1.67$ fitting that of one of our least damaged weld specimen. The electric transfer resistance of a square-electrode ACPD probe was calculated for two orthogonal directions as the probe was moved across the bondline from the weld to the base metal. The potential drop values obtained from these numerical simulations were used to calculate the normalized average resistance and anisotropy according to Eqs. (61) through (62). Figures 10(a) and 10(b) show a comparison of the analytical and numerical results for the normalized average resistance and normalized anisotropy, respectively. The finite element results agreed with the analytical predictions within a small numerical error related to the meshing density used. In the approximation of our simple idealized model of the weld/base metal interface, the average resistance gradually increases from the base metal, through the interface to the weld and the apparent anisotropy becomes significant only in the close vicinity of the bondline between the weld and the base metal.

a)

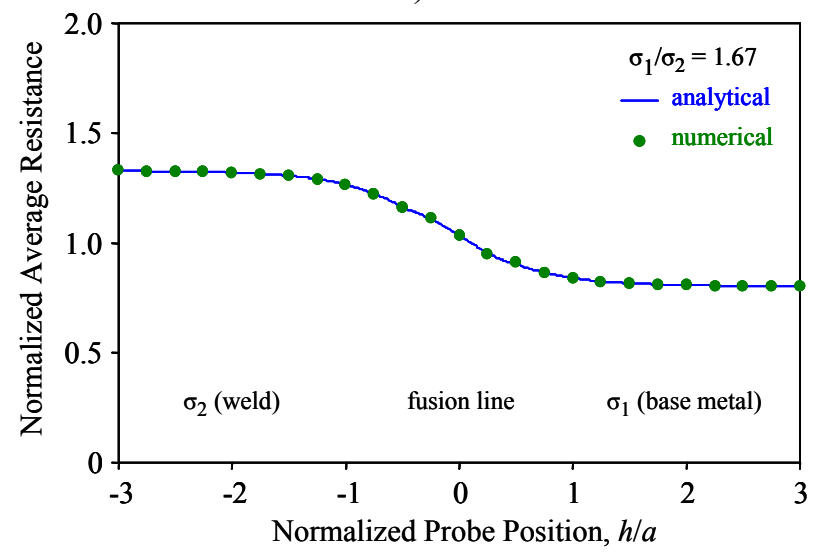

b)

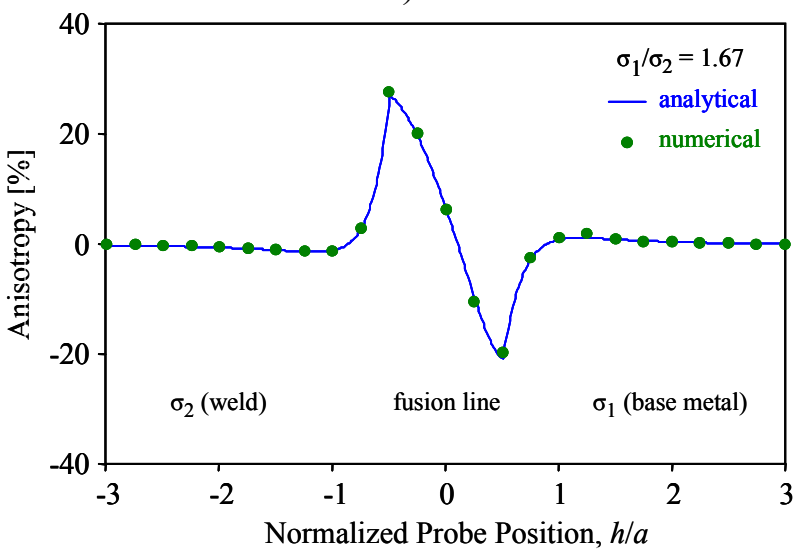

Fig. 10 Comparison between the analytical and numerical results for the normalized average resistance (a) and anisotropy (b) as a function of probe position for a normal interface.

From these results it can be concluded that the apparent anisotropy near the bondline is caused by the sudden change in conductivity between the base metal and the weld region and has nothing to do with the potential presence of creep damage in the same area. This apparent anisotropy that is observed only in the vicinity of the weld/base metal boundary renders the detection of any creep damage near this boundary more difficult. The exact match between the analytical and numerical simulations for the normalized apparent anisotropy is due to the consideration of a perfectly normal $\left(90^{\circ}\right)$ interface in the numerical simulation in agreement with the analytical model. In contrast, when comparing to experimental results we do not expect such perfect agreement with the analytical predictions. This discrepancy could be due to the inclination of the interface since the boundary between the weld and the base metal is usually not exactly normal to the surface. Another possible source of discrepancy between the theoretical 
model and the experimental test piece is the gradual rather than abrupt conductivity transition between the weld and the base metal. These two secondary effects are difficult to study analytically, but could easily be investigated by numerical means.

\subsection{Boundary inclination and gradual transition}

Comparisons between these analytical and numerical predictions and experimental results to be shown later indicate that the observed underestimation in the measured anisotropy could be due to the random offset uncertainty caused by probe positioning as it is placed over the bondline between the weld and the base metal. Even with perfect probe positioning relative to the bondline on the surface, significant additional uncertainty could be caused by the unknown inclination of the interface relative to the surface and/or by the gradual transition between the weld and the base metal. Figure 11 shows schematic diagrams of imperfect weld/base metal models for finite element simulation of boundary inclination (a) and gradual transition (b).

a)

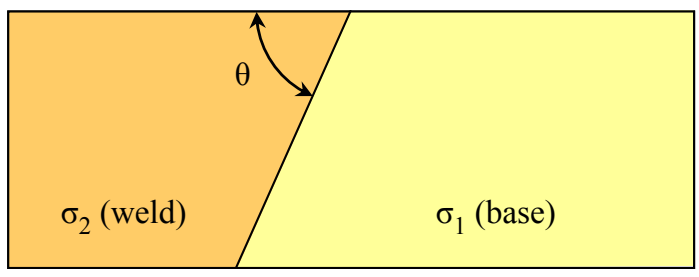

Fig. 11 Imperfect weld/base metal models for finite element simulation of boundary inclination (a) and gradual transition (b).

In order to study the effect of boundary inclination relative to the surface, numerical simulations were conducted at seven different boundary inclination angles. The simulation was performed the same way as before except that the boundary was not necessarily normal to the surface. Figure 12(a) shows the normalized average resistance as a function of the normalized probe position at seven different boundary inclination angles. Changing the boundary inclination angle caused only a minor shift in the normalized average resistance curves. This could be expected since the penetration depth of the measurement is roughly equal to only half of the electrode separation therefore the effective position of the boundary is shifted only by a small amount relative to the visible bondline on the surface depending on the inclination angle. Figure 12(b) shows the apparent anisotropy as a function of the normalized probe position at the same seven boundary inclination angles. Changing the inclination angle somewhat reduced the apparent anisotropy on both sides of the weld/base metal boundary depending on the inclination angle but did not eliminate it. This effect could be also expected since the inclined interface appears as a slightly shifted gradual transition and the location of the actual bondline is a function of depth therefore the probe averages over its effective penetration depth.

Gradual transition in the electric conductivity near the interface region was also studied using finite element analysis. In order to minimize the number of adjustable parameters in the finite element simulation, the transition layer was assumed to be of constant width $t=a / 2$ equal to half of the electrode separation. Linear interpolation was used to distribute the conductivity difference from weld to base metal and the number $n$ of homogeneous sub-layers was varied 
from 0 to 4 to represent transition profiles of different smoothness. Figure 13(a) shows the normalized average resistance for the four different transition profiles as a function of the normalized probe position. The average resistance did not show any perceivable sensitivity to the smoothness of transition and the gradual increase from the base metal through the interface to the weld region was essentially the same for each of the four cases studied. In contrast, the 4step smoothened transition layer significantly reduced the apparent anisotropy as shown in Figure 13(b), though did not completely eliminate it. These results suggest that gradual transition of the electric conductivity throughout the fusion boundary layer between the weld and the base metal is more likely to lead to reduced apparent anisotropy than non-normal inclination of the interface relative to the surface.

a)

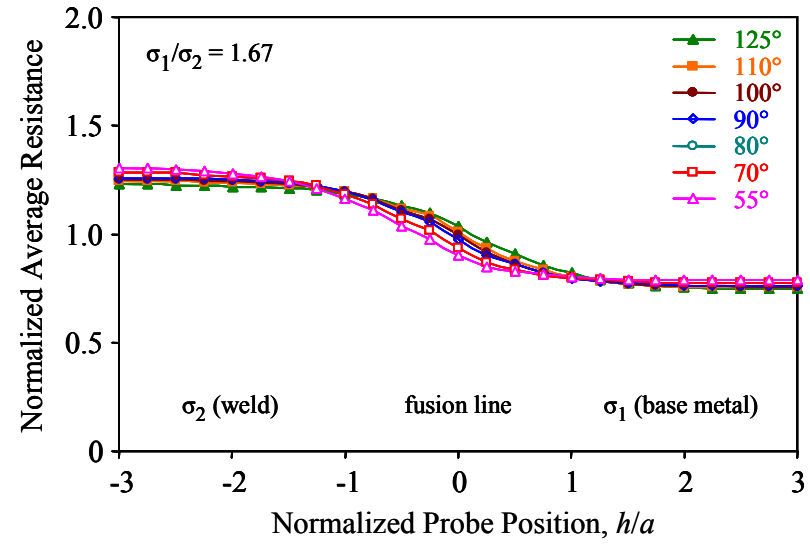

b)

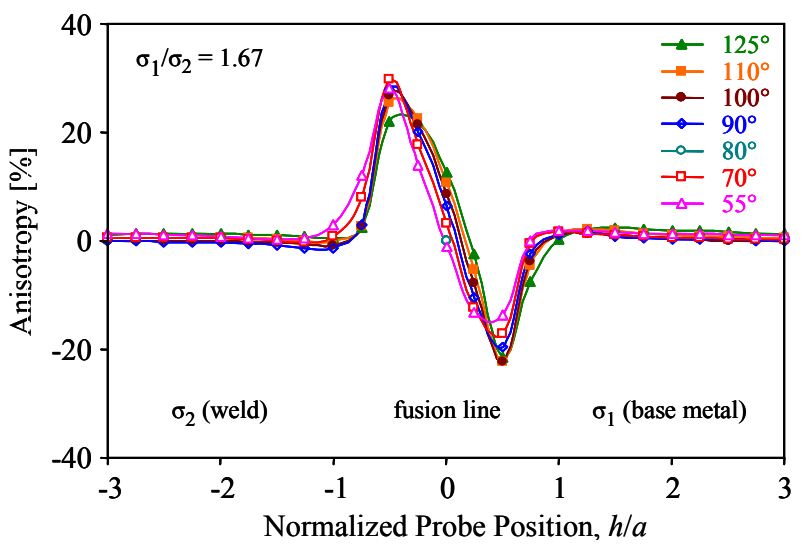

Fig. 12 Finite element predictions for the normalized average resistance (a) and anisotropy (b) for seven different boundary inclination angles.

a)

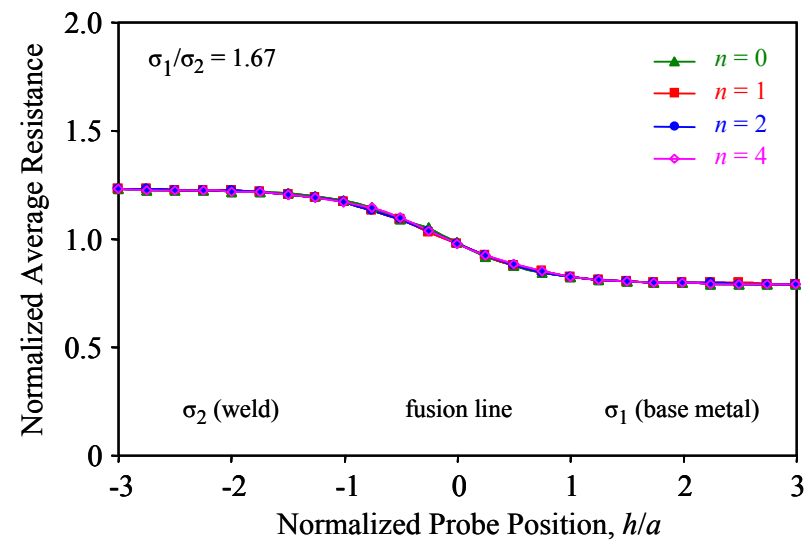

b)

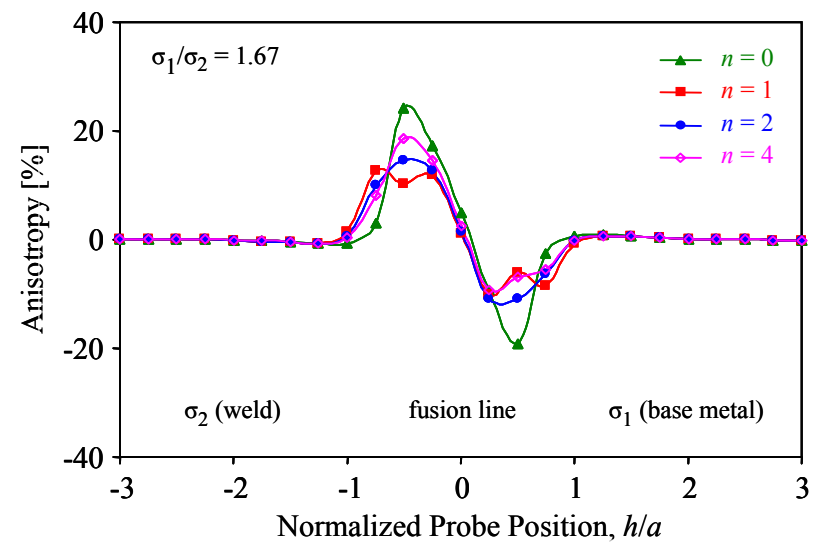

Fig. 13 Finite element predictions for the normalized average resistance (a) and anisotropy (b) for four different boundary models. 


\section{DACPD Creep Assessment with Deployable Electrodes}

In an exploratory test, electrical anisotropy measurements were performed in three regions (base metal, interface zone, and weld region) on four welded specimens cut from a large $0.5 \% \mathrm{Cr}$ $0.5 \% \mathrm{Mo}-0.25 \% \mathrm{~V}$ low-alloy steel pipe using the above described deployable DACPD probe [50]. All samples were sections of headers with welds, which had been tested by replica examination after operating for about 150,000 hours at $570{ }^{\circ} \mathrm{C}$ and 165 bars. Replica analysis invariably found that more cavities were present in the heat affected zone (HAZ) than in the region farther away from the weld. Table 1 lists the different cavity density levels measured by replica inspection in the HAZ of these four specimens.

Table 1 Different cavity density levels in the tested four creep-damaged specimens.

\begin{tabular}{|c|c|}
\hline Specimen & Cavity Density $\left[\mathbf{m m}^{-2}\right]$ \\
\hline$\# 1$ & 1200 \\
\hline$\# 2$ & 300 \\
\hline$\# 3$ & 150 \\
\hline$\# 4$ & 45 \\
\hline
\end{tabular}

Five sets of DACPD measurements were taken with twenty-five repetitions averaged per location in the three regions as shown in Figure 14 (sample \#2). As expected, the average resistance monotonically increased from the base metal, through the interface, to the weld region as shown in Figure 15(a). The error bars indicate the standard deviation of the measurement and include the measurement repeatability as well as random point to point variations parallel to the bondline. The resistivity anisotropy calculated between the normal and tangential directions relative to the weld boundary was low in both the base metal and the weld regions and it peaked in the interface region as shown in Figure 15(b).

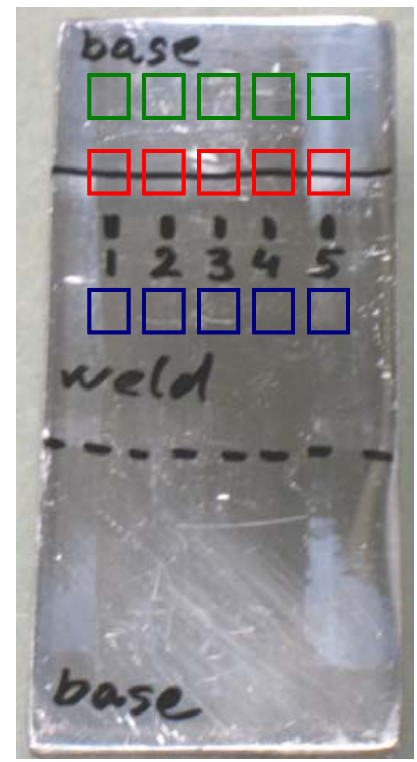

Fig. 14 Welded specimen \#2 with five measurement locations $(1,2, \ldots .5)$ marked in three regions (base metal, the interface and the weld). 
a)

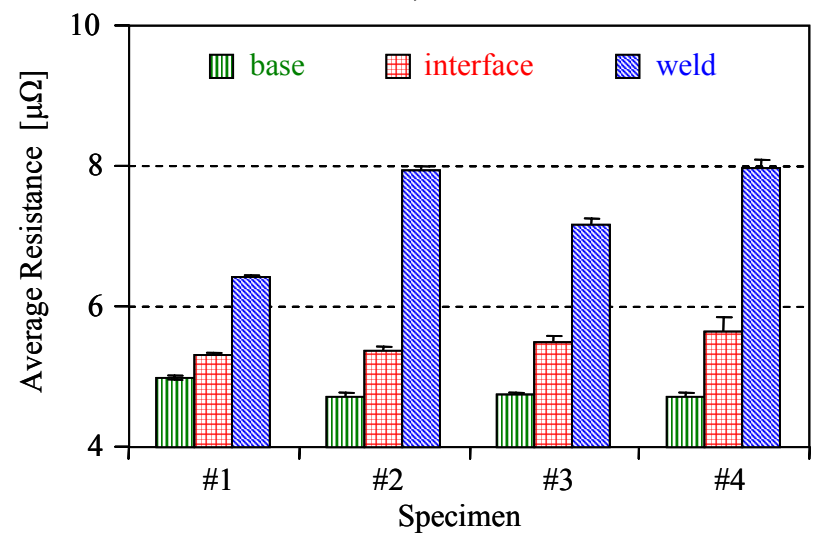

b)

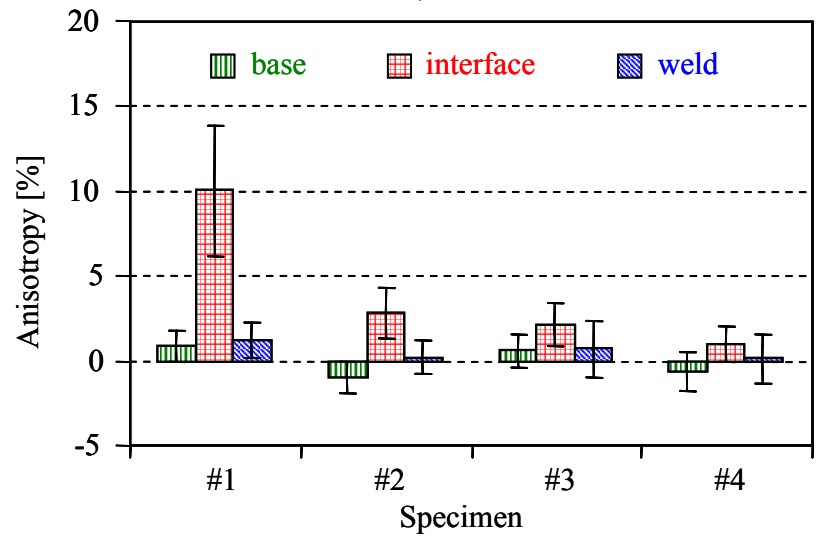

Fig. 15 The measured average resistance (a) and anisotropy (b) of four creep damaged weld specimens in three regions.

The above results indicate that the increase in electric anisotropy correlates well with the creep damage location. At the same time, there is a much higher than expected level of experimental variation in the data that cannot be explained by random electric noise and electrode positioning uncertainties. This added uncertainty occurs mainly in the interface region separating two regimes of different electric conductivity. The increased level of variability in the interface zone can be readily explained by the above discussed results of our combined analytical/computational effort to better understand the nature of this variation. As an example, Figure 16 shows a comparison between the analytical predictions and the experimental results for the least damaged specimen (\#4) we had access to. This specimen had negligible creep damage $\left(45\right.$ cavities $\left./ \mathrm{mm}^{2}\right)$ but very large conductivity contrast $\left(\sigma_{1} / \sigma_{2}=1.67\right)$.

a)

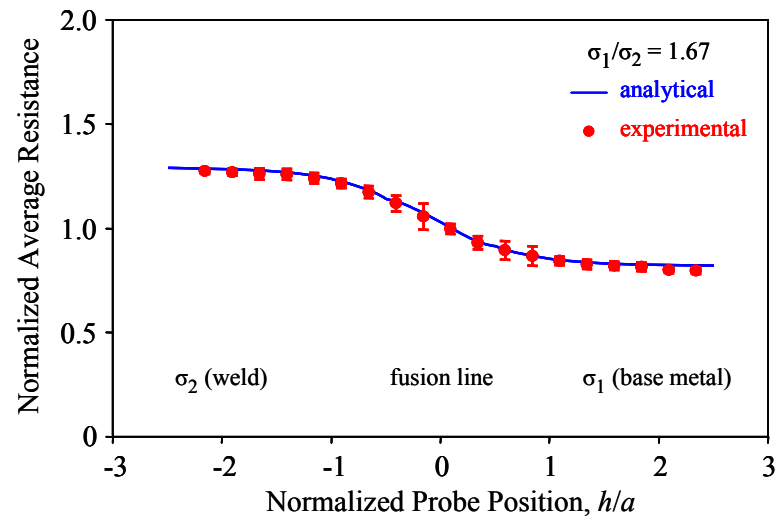

b)

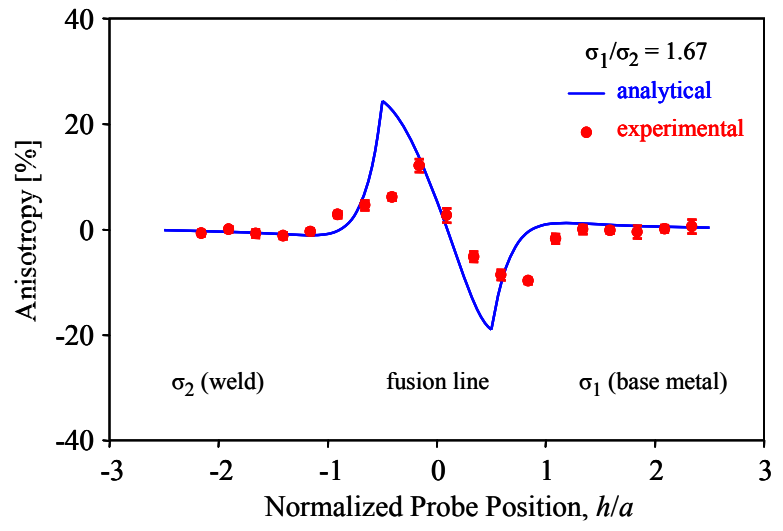

Fig. 16 Comparisons between analytical (normal interface) and experimental results from specimen \#4 of negligible creep damage for the normalized average resistance (a) and anisotropy (b) as a function of probe position (specimen \#4).

It should be pointed out that the actual offset from the perceived bondline could have been off by as much as $\Delta h=+/-0.5 \mathrm{~mm}$ while the electrode separation was only $a=4 \mathrm{~mm}$, therefore the normalized offset uncertainty could be as high as $\Delta h / a=+/-12.5 \%$ which is enough to cause $+/-8 \%$ uncertainty in the measured apparent anisotropy. Considering the inevitable uncertainty 
in probe positioning caused by the irregularity of the weld boundary, the experimental results agreed with the analytical predictions quite well for both the normalized average resistance shown in Figure 16(a) and normalized anisotropy shown in Figure 16(b). On the other hand, even with careful probe positioning, the measured anisotropy tends to be lower than expected because the interface between the weld and the base metal is not necessarily normal to the surface and the transition is not necessarily as sharp as assumed in the analytical model, two secondary effects that were addressed in the previous section.

From the comparison between the analytical, experimental and numerical results it can be concluded that the apparent anisotropy near the bondline is caused by the sudden change in conductivity between the base metal and the weld region and has nothing to do with the potential presence of creep damage in the same area. This apparent anisotropy that is observed only in the vicinity of the weld/base metal boundary makes any creep damage concentrated at this boundary more difficult to detect. The exact match between the analytical and numerical simulations for the normalized apparent anisotropy previously shown in Figure 10 was due to the consideration of a perfectly normal interface in the numerical simulation in agreement with the analytical model. In contrast, the experimental results, though they exhibit similar anisotropic behavior, do not overlap with the analytical predictions partly because of the inclination of the interface and partly because of the gradual rather than abrupt conductivity transition between the weld and the base metal.

\subsection{Effect of thermal evolution of the microstructure}

Further tests were conducted to study the effect of thermal exposure on the average resistance and intrinsic anisotropy of the weld and base metals separately. The importance of these tests lies in the role of changing anisotropy during creep. In creep monitoring with either permanently installed or deployable DACPD probes, the measured resistance ratio is normalized to the initial resistance ratio recorded in the intact state of the material to eliminate systematic errors caused by imperfect electrode positioning and pre-existing texture. Any further change in the resistance ratio is then interpreted as either creep-induced geometrical effect due to plastic strain (only in the case of permanently installed electrodes) or material effect in the form of creep-induced directional damage leading to electric anisotropy. An obvious concern in this respect is that small changes in the apparent or real material anisotropy might occur as a result of thermallyactivated microstructural evolution even in the absence of stress, i.e., actual creep.

In more or less homogeneous materials, i.e., far away from weld/base metal interfaces, the only issue is whether the intrinsic electric anisotropy of the material might change due to thermal exposure. The intrinsic electric anisotropy of creep resistant steels is limited to morphological texture and is usually very weak, less than $1 \%$, therefore this effect is not expected to be significant. However, the apparent anisotropy measured in the vicinity of weld/base metal boundaries could exhibit much more significant changes because the resistivity of the weld and base metals could change at very different rates during thermal exposure, which influences their resistance ratio and therefore the level of apparent anisotropy for a given probe position, especially if the probe straddles the boundary, but is not centered directly over it.

This effect was studied separately in specimens cut from the weld and base metal parts of a $0.5 \% \mathrm{Cr}-0.5 \% \mathrm{Mo}-0.25 \% \mathrm{~V}$ low-alloy steel pipe. Before thermal exposure, the resistivity of the weld was found to be as much as $75 \%$ higher than that of the base metal. Thermal exposure tests were conducted subsequently at three different temperatures, namely at $650{ }^{\circ} \mathrm{C}, 700{ }^{\circ} \mathrm{C}$ and $750{ }^{\circ} \mathrm{C}$. At each temperature, the base metal and weld were heated to the test temperature in five subsequent cycles of 3 hours, 7 hours, 20 hours, 70 hours and 200 hours. Between the cycles, 
i.e., after 3,10, 30, 100, and 300 hours of combined thermal exposure, the electric resistances were measured in two orthogonal directions with the previously described deployable DACPD probe. The relative anisotropy and average resistance were calculated from these measured resistance pairs. After the full five-step heat treatment cycle was completed at one temperature, the temperature was increased to the next level and the cycle was repeated. Overall, the two samples were heat treated for a total of 900 hours with 300 hours at each temperature.

During the whole series of thermal exposure tests, the intrinsic anisotropy of both the weld metal and the base metal remained essentially constant at $\Delta \approx 1 \%$, which was barely detectable in the presence of comparable measurement uncertainties. The normalized average resistances measured in every heat treatment step on the two samples are plotted in Figure 17. The symbols represent measurement data and the solid, dashed and dotted lines are linear regressions (they look exponential because of the logarithmic time scale). The averaged values of the electric resistances were normalized to those of the corresponding intact (initial state) samples for easier comparison. The average resistance for both the base metal and weld exhibited some increase during thermal exposure. However, in the weld metal the changes were barely noticeable at $650{ }^{\circ} \mathrm{C}$ and $700{ }^{\circ} \mathrm{C}$ and even at $750{ }^{\circ} \mathrm{C}$ remained below $0.6 \%$ total increase. Much larger changes occurred in the base metal. Initially, the resistance slightly decreased at $650{ }^{\circ} \mathrm{C}$ but then started to increase at $700{ }^{\circ} \mathrm{C}$ and reached as much as $6.6 \%$ higher at the end of the 300 -hour exposure at $750{ }^{\circ} \mathrm{C}$. This means that high-temperature thermal exposure will reduce the resistivity difference between the weld and the base metals, at least at peak temperatures. It should be mentioned that the average operational temperature for this kind of steel is expected to be around $600{ }^{\circ} \mathrm{C}$ or less, therefore the thermal exposure used in this example was relatively short, but still quite excessive because of the $750{ }^{\circ} \mathrm{C}$ peak temperature.

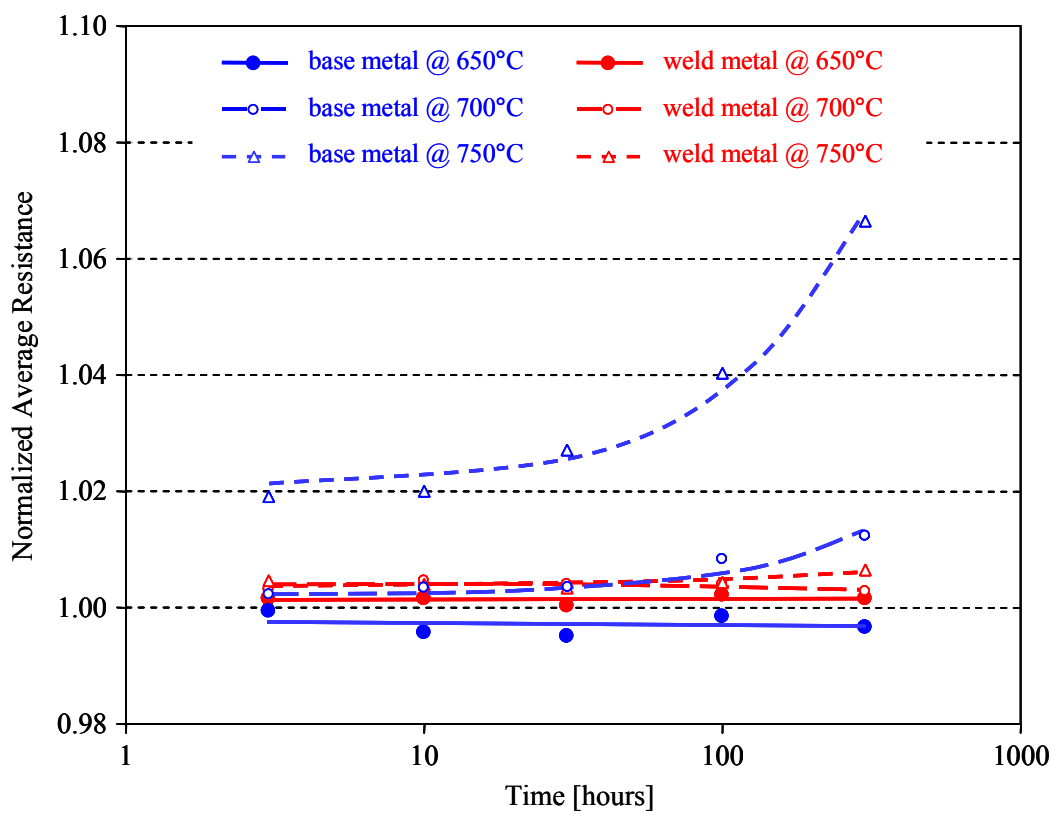

Fig. 17 Normalized average resistance of the base metal and weld throughout three different heat treatment cycles. The solid, dashed and dotted lines are linear regressions.

According to our earlier results, in the worst case scenario, i.e., when the offset of the probe from the weld/base metal boundary is the least favorable, $6.6 \%$ change in the resistivity contrast will cause approximately $2.6 \%$ spurious change in the measured resistance ratio. This change 
(reduction) in the apparent anisotropy measured by the DACPD probe exceeds all other experimental uncertainties, therefore will adversely affect the sensitivity of creep monitoring by this technique. However, in the case of permanently mounted probes, the combined geometrical and material gauge factors produce changes on the order of $200-300 \%$ in the resistance ratio before rupture, therefore the role of apparent anisotropy remains limited even in the vicinity of weld/base metal boundaries.

In the case of deployable DACPD probes the geometrical effect of creep strain is eliminated therefore material changes, including both real anisotropy changes due to creep damage accumulation and apparent anisotropy changes in the vicinity of the weld due to unrelated thermally-induced microstructural evolution, become dominant. The spurious apparent anisotropy can be minimized by aligning the probe with the weld/base metal boundary as suggested by the zero crossings in Figure 16(b) and also by our numerical simulations. In practice, deployable probes are not expected to reach much better than $+/-1 \%$ repeatability on perfectly isotropic materials even with precision-guided electrode pins. Compared to this inherent targeting uncertainty, the influence of apparent anisotropy changes due to thermal evolution can be all but eliminated by careful positioning of the probe over the weld/base metal boundary. One possibility is to measure the average resistances of the base metal and the weld far away from the boundary and then optimize the position of the probe so that the average of the two resistivity values measured normal and parallel to the bond line matches the algebraic means of the weld and base metal resistances.

\subsection{Electric properties of 9Cr-1Mo steel}

We also used the deployable DACPD probe to establish the electric conductivity and anisotropy of the Grade 91 9Cr-1Mo steel plates provided by Idaho National Laboratory for the purposes of this project as shown in Figure 18. Figure 19 shows the electric resistance (a) and anisotropy (b) of Grade 91 9Cr-1Mo steel measured by a 4-mm square-electrode DACP probe at $2 \mathrm{~Hz}$. The average electric resistance was found to be $11.82 \mu \Omega$ with a modest $0.18 \mu \Omega(\approx 1.5 \%)$ scatter over the inspected five locations. Based on the electrode separation, the electric resistivity can be calculated from Eq. (18) as $\rho=507 \mu \Omega \mathrm{mm}(\approx 3.4 \%$ IACS $)$, a rather low value but well in line with published data for this material [51]. Because of this relatively high electric resistivity, the standard penetration depth at $2 \mathrm{~Hz}$ is as high as 20-25 $\mathrm{mm}$ depending on the magnetic permeability of the material.

This is very important because the magnetic permeability of ferromagnetic steels is usually highly variable and very strongly influenced by such spurious effects as prior magnetization, the presence of residual and applied stresses, subtle microstructural changes, temperature, etc. The cut-off frequency $f_{\mathrm{c}}$ above which the real part of the AC impedance of the material is strongly affected by permeability variations can be estimated from the condition that the standard penetration depth $\delta$ is approximately equal to the electrode separation $a$. For example, in the case of a square-electrode probe of $a=4 \mathrm{~mm}$ on G91 steel, this cut off frequency is about $f_{\mathrm{c}}=80$ $\mathrm{Hz}$ at room temperature and drops to $f_{\mathrm{c}}=13 \mathrm{~Hz}$ if a larger $a=10 \mathrm{~mm}$ probe is used to increase the penetration depth of the measurement. It should be mentioned, that the resistivity increases with temperature and at $650{ }^{\circ} \mathrm{C}$ the resistivity is higher by more than a factor of two so that the cut-off frequency is higher by a factor of four. Still it is important to realize, especially when working with materials of lower electric resistivity that the inspection frequency must be chosen to be well below the cut-off frequency to avoid spurious interference of the material's magnetic behavior with AC electric resistance measurements. 


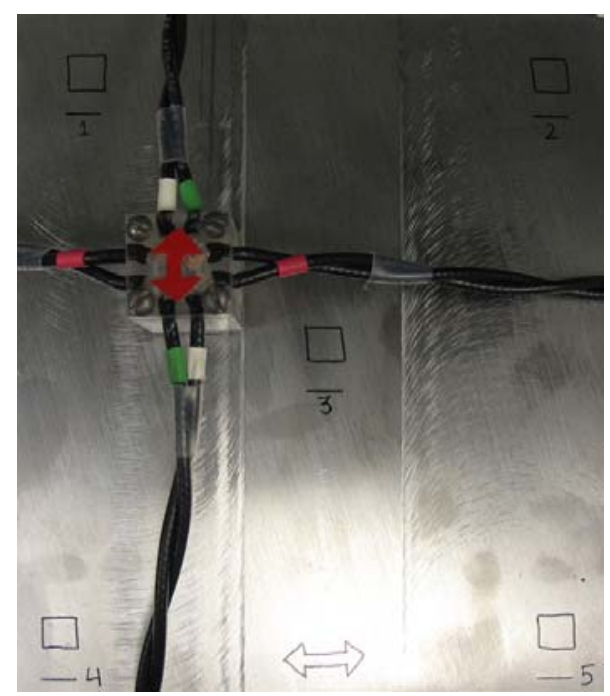

Fig. 18 Image of the deployable DACPD probe used to measure the electric conductivity and anisotropy of the Grade 91 9Cr-1Mo steel plate provided by Idaho National Laboratory. The five numbered squares indicate the measurement locations while the arrow shows the rolling direction of the plate.

The same measurements were also evaluated for the local anisotropy of the electric resistivity in the tested Grade 91 9Cr-1Mo steel plate. As it is shown in Figure 19(b), the measured relative anisotropy between the rolling and transverse directions was essentially negligible with respect to the roughly $\pm 1.3 \%$ random variation of the data. In the absence of directional creep damage, the lack of electric resistivity anisotropy is expected even in highly textured polycrystalline materials of cubic symmetry and our results are consistent with this expectation.

a)

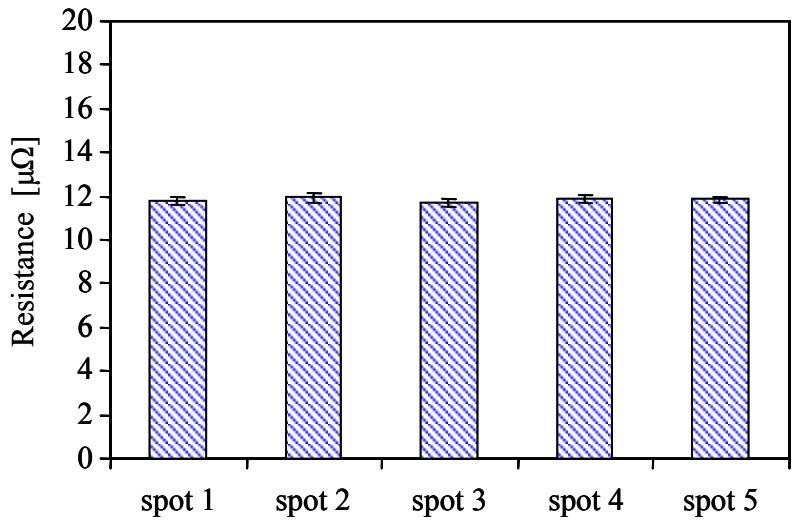

b)

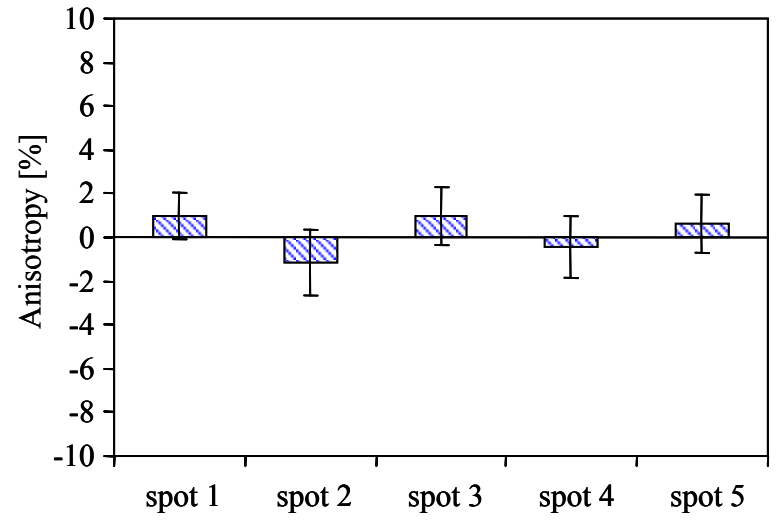

Fig. 19 Electric (a) resistance and (b) anisotropy of Grade 91 9Cr-1Mo steel measured by a 4-mm square-electrode DACPD probe at $2 \mathrm{~Hz}$.

\section{DACPD Creep Monitoring System with Permanently Installed Electrodes}

In this section we introduce the first generation DACPD system we developed for in-situ creep monitoring and validate our previously presented analytical predictions. Based on these initial tests, we also developed a second generation DACPD instrumentation that is specifically 
optimized for long-term creep tests on G91 steel. In the rest of this section we report on our extensive laboratory tests on G91 base metal and weld material as well as present some preliminary results obtained by our collaborators on a Gleeble thermo-mechanical simulator machine at the Idaho National Laboratory.

\subsection{First generation DACPD system and validation tests}

The first generation directional alternating current potential drop (DACPD) measurement system exploited the uniquely high accuracy of low-frequency ACPD techniques to monitor resistance changes in real time during thermo-mechanical exposure of the material. The DACPD creep monitoring system uses a pair of injection electrodes and a separate pair of sensing electrodes to measure the electrical impedance of a conducting material. Figure 20 shows how these electrodes are either spot welded to the specimen to be monitored or to standard electrodes of slightly larger diameter that have been stud-welded to the specimen for field tests requiring more rugged electrodes. In low-frequency ACPD measurements the so-called "skin" effect is negligible, i.e., the injection current spreads through the whole cross section of the specimen limited only by the electrode separation, like in the case of Direct Current Potential Drop (DCPD) measurements, while the phase-locked detection greatly increases the measurement accuracy.

a) spot-welded wires

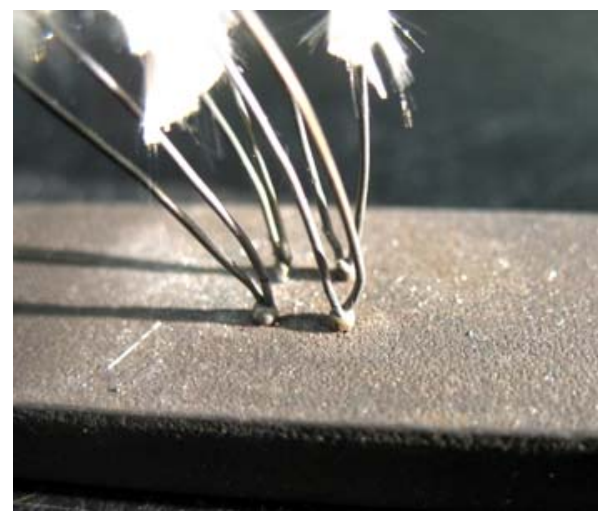

b) stud-welded electrodes

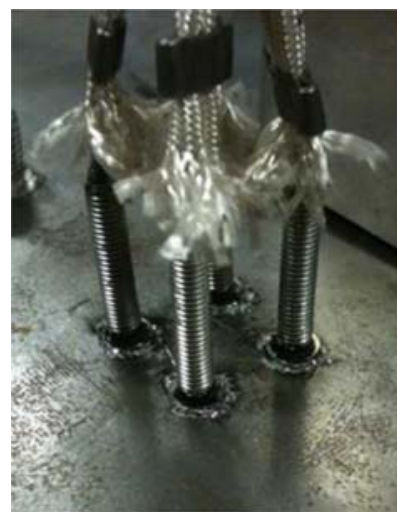

Fig. 20. Permanently attached electrodes for DACPD creep monitoring.

The block diagram of the first generation electronic instrumentation is shown in Figure 21. To summarize the working principle of the system, a signal is generated by the internal oscillator of the lock-in amplifier and passes through a differential output amplifier. The purpose of the latter is to minimize the common mode signal of the specimen since otherwise even the best common mode rejection ratio we can reach (around $140 \mathrm{~dB}$ ) would be insufficient to make sufficiently accurate measurements. Then the current goes through a non-contacting magnetic sensor to measure its actual value in order to compensate during data processing for any change in the current level due to variations in the resistance of the multiplexer's relays or in the wiring and contact resistances. The multiplexer's main role is to switch between electrode configurations in order to obtain the two sought resistance values in the axial and lateral directions and to allow multiple-site monitoring. In addition, the multiplexer allows us to monitor creep evolution essentially simultaneously at more than one location if necessary. The voltage measured between the sensing electrodes is fed first through a 1:8 high-impedance transformer, then through a 100-gain preamplifier, and then read with the lock-in amplifier. 
The quantity measured in potential drop tests is a voltage difference and the measurement results are presented in the form of transfer resistance which is taken as the real part of the ratio between the voltage difference $\left(V^{+}-V^{-}\right)$between the sensing electrodes and the value of the injected current. It should be mentioned that it was essential to optimize the sensor not only to maximize the signal-to-noise ratio but also to accommodate the wide range of magnetic permeability values inherent to power plant steels. This experimental system offers excellent reproducibility and accuracy by operating at sufficiently low inspection frequency so that the standard penetration depth is always larger than the electrode separation of $4 \mathrm{~mm}$. Under such conditions, this low-frequency ACPD instrument operates effectively as a DCPD technique that is inherently insensitive to inevitably large variations in magnetic permeability.

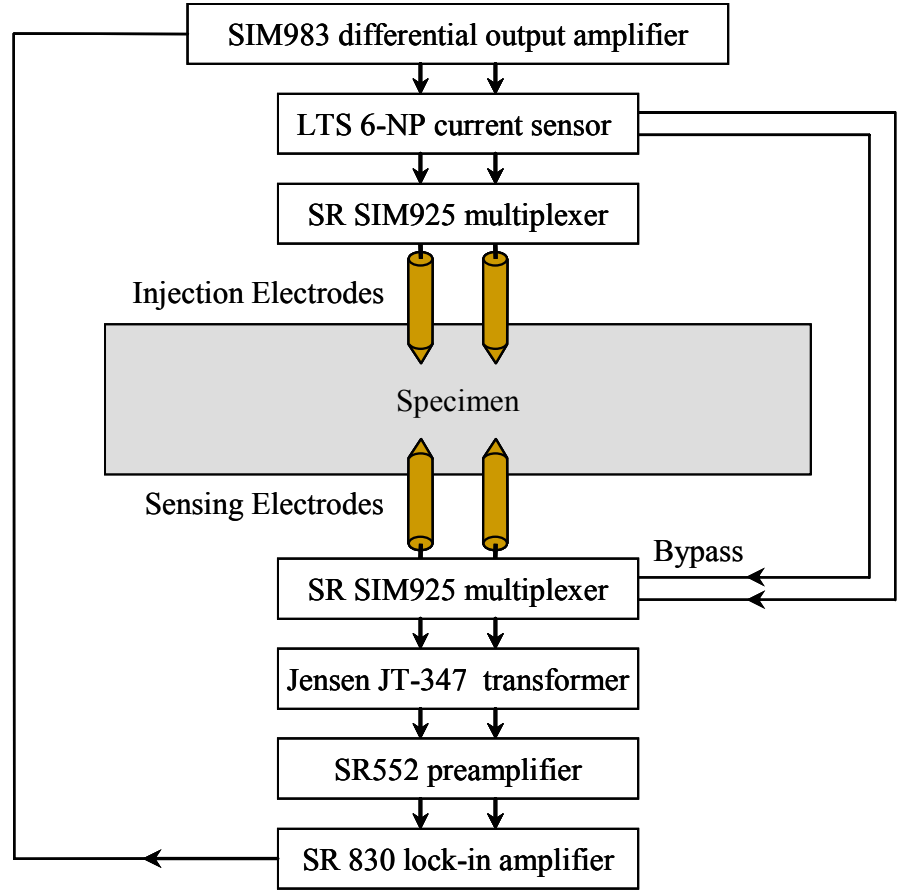

Fig. 21. A schematic diagram of the first generation electronic measurement system.

The above described low-frequency ACPD system is capable of accurate measurements down to $1 \mathrm{~Hz}$ which is less than what is necessary even for ferritic steels especially for small electrode separation $a \approx 4 \mathrm{~mm}$. An important parameter to take into consideration when using low-frequency ACPD measurements for creep monitoring is the initial geometrical aspect ratio $\gamma_{0}=a_{10} / a_{20}$ of the electrode configuration. In practice, it is very difficult to assure that the spot welded electrodes are uniformly spaced in an ideal square-configuration. Fortunately, the earlier described initial normalization effectively suppresses spurious resistance variations caused by mounting errors. However, large initial aspect ratios $\gamma_{0}=a_{10} / a_{20}$ present a problem in accurate resistivity ratio measurements. It was found that a $+/-10 \%$ relative positioning error between the electrodes, i.e., only $+/-0.4 \mathrm{~mm}$ for $4 \mathrm{~mm}$ nominal electrode separation, might cause as much as $50 \%$ difference in the initial values of $R_{10}$ and $R_{20}$, which causes practical difficulties in maintaining the measurement accuracy necessary for creep monitoring. Therefore, it is of great importance to keep the uncertainty in electrode positioning to a strict minimum. 


\subsection{System validation}

In order to increase the measurement's selectivity to directional variations due to creep, the sensor must exhibit low sensitivity to non-directional reversible thermal effects. To investigate this issue, the sensor was tested on 304 stainless steel by exposing it to a cumulative 252-hour heating regime at a temperature of $500{ }^{\circ} \mathrm{C}$. Figure 22(a) shows the average normalized resistance $\bar{\eta}=\left(\eta_{1}+\eta_{2}\right) / 2$ and temperature $T$ as functions of test time during a reversible sixcycle thermal exposure of 304 stainless steel at $500{ }^{\circ} \mathrm{C}$ peak temperature and Figure 22(b) shows the resulting variation in the normalized resistance ratio $\xi$. Due to the high average thermal coefficient of electric resistivity $\left(720 \mathrm{ppm} /{ }^{\circ} \mathrm{C}\right)$ in 304 stainless steel, the "common" normalized resistance exhibits an increase of about $45 \%$ while the accompanying change in $\xi$ is less than $\pm 0.17 \%$. The relatively small changes observed in resistance ratio are likely to be due to initial cold work in the material that led to subsequent directional microstructural changes during thermal exposure. Overall, the observed variations in $\bar{\eta}$ can be considered mostly reversible since there was practically no remnant change at room temperature. Let us introduce the thermal common mode rejection ratio TCMRR $=\Delta \bar{\eta} / \Delta \xi$ of the sensor to characterize its ability to suppress changes common to both axial and lateral resistance measurements due to thermal effects. For the reversible thermal effects on 304 stainless steel, the TCMRR typically reaches values higher than 250 . This high level of TCMRR indicates that the creep sensor can efficiently suppress the very large reversible resistance variations due to wide temperature changes expected during in-situ creep monitoring.

a)

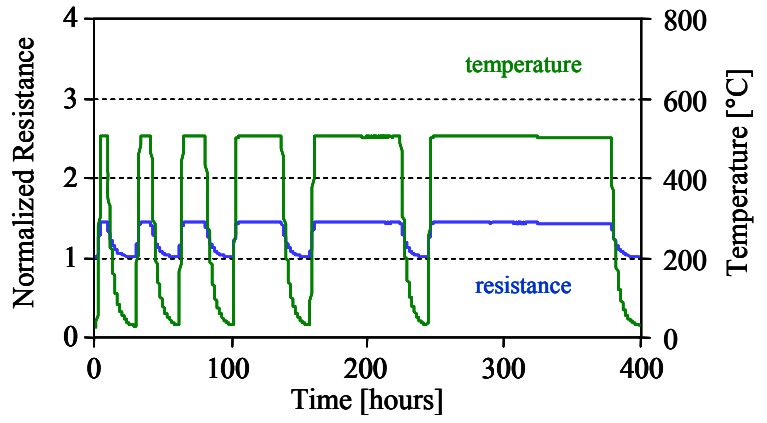

b)

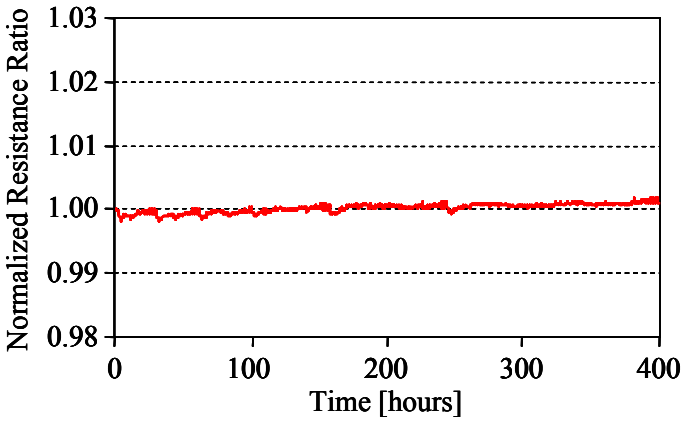

Fig. 22. Normalized average resistance (a) and normalized resistance ratio (b) variations during reversible thermal exposure of 304 stainless steel at $500^{\circ} \mathrm{C}$.

Overall, the irreversible TCMRR seems to be a little lower and strongly material dependent, but a value of $\approx 10$ is usually achieved even in specimens that are strongly textured in their initial state. However, the irreversible thermal changes in $R_{1,2}$ are not expected to exceed $10 \%$, therefore the corresponding effect on $\xi$ is around $1 \%$, similar to the much larger, but better suppressed reversible thermal effects. Figure 23(a) shows the average normalized resistance ratio $\bar{\eta}$ and temperature $T$ as functions of test time during an irreversible four-cycle thermal exposure of 304 stainless steel bar stock at $650{ }^{\circ} \mathrm{C}$ and Figure 23(b) shows the resulting variation of the normalized resistance ratio $\xi$. At this elevated temperature, the normalized resistance ratio drifted as much as $0.6 \%$ and might exhibit an additional $0.3 \%$ reversible oscillation between room temperature and $650{ }^{\circ} \mathrm{C}$. This small yet perceivable irreversible thermal effect seems to be an intrinsic feature of the sensor on specimens exhibiting strong initial manufacturing texture. However, it represents only a small impact on the overall performance of the sensor thanks to the 
high sensitivity of $\xi$ to creep strain as was shown earlier. In effect, $1 \%$ plastic strain causes $\approx 5 \%$ change in $\xi$, therefore $0.6 \%$ irreversible thermal change in the normalized resistance ratio represents a very low $\approx 0.1 \%$ detection threshold in terms of creep strain.

a)

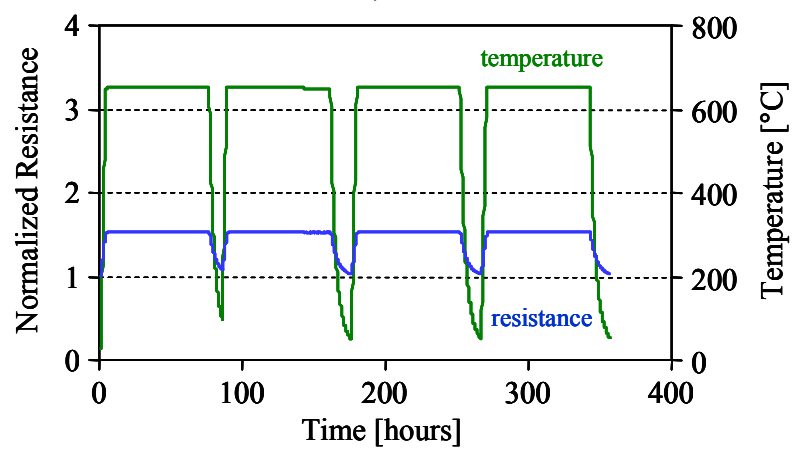

b)

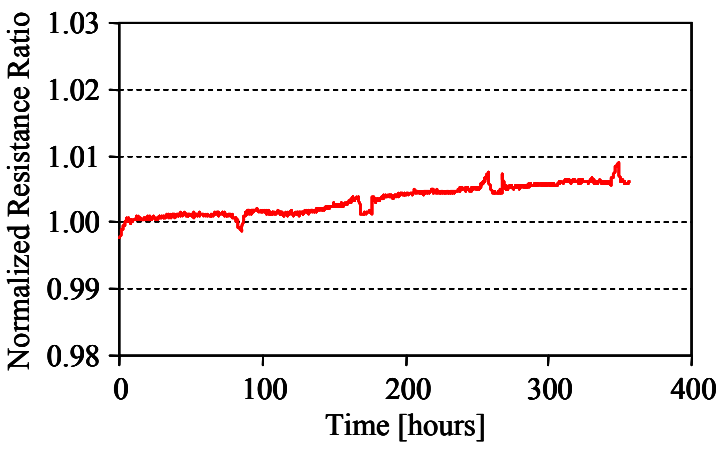

Fig. 23. Normalized average resistance (a) and normalized resistance ratio (b) variations during irreversible thermal exposure of 304 stainless steel at $650{ }^{\circ} \mathrm{C}$.

The creep monitoring sensor was first tested on 304 stainless steel under elastic and plastic strain conditions at room temperature. Figure 24 shows smoothened ACPD resistance measurements at $3 \mathrm{~Hz}$ with a square-electrode sensor in the (a) elastic and (b) plastic regimes of 304 stainless steel at room temperature. In the elastic regime alternating axial load was applied to the specimen at a cyclic frequency of $0.01 \mathrm{~Hz}$. The maximum tensile load resulting in almost $0.15 \%$ elastic strain was chosen to be about twice as high as the maximum compressive load in order to minimize the possibility of buckling in the slender rectangular bars $(177.80 \times 25.40 \times 6.35$ $\mathrm{mm})$.

a)

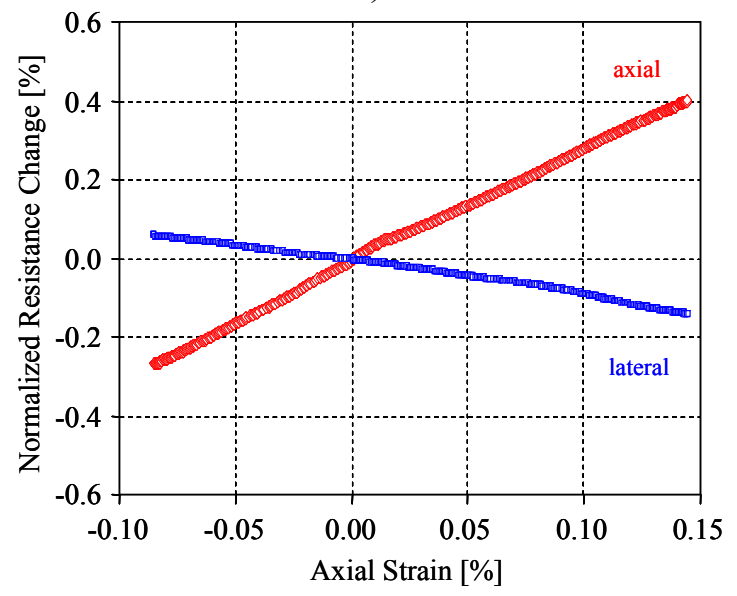

b)

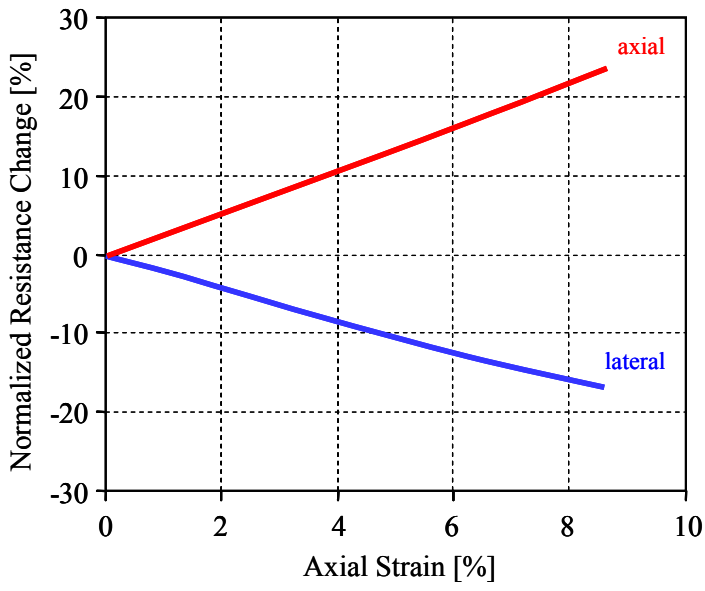

Fig. 24. Smoothened ACPD resistance measurements with a square-electrode sensor in the (a) elastic and (b) plastic regimes of 304 stainless steel at room temperature $(3 \mathrm{~Hz})$.

The results yield values of $f_{1} \approx 2.95$ and $f_{2} \approx-0.86$ for an overall elastic geometrical gauge factor of $f \approx 3.81$, which is within reasonable agreement with the theoretical bounds (3.75-4.44) calculated earlier. For the plastic regime, specimens were subjected to gradually increasing uniaxial tensile stress up to failure which occurs at $\approx 10 \%$ strain in 304 stainless steel at room temperature. Figure 24(b) shows an example of normalized resistance change versus plastic 
strain. The measured values were $F_{1} \approx 2.74$ and $F_{2} \approx-1.96$ for an overall plastic geometrical gauge factor of $F \approx 4.70$, which is also in agreement with the theoretical bounds (4.33-5.12) calculated earlier. In summary, Figure 25 illustrates the acceptable agreement between the experimentally determined values of the elastic and plastic geometrical gauge factors for several specimens and their respective theoretical bounds.

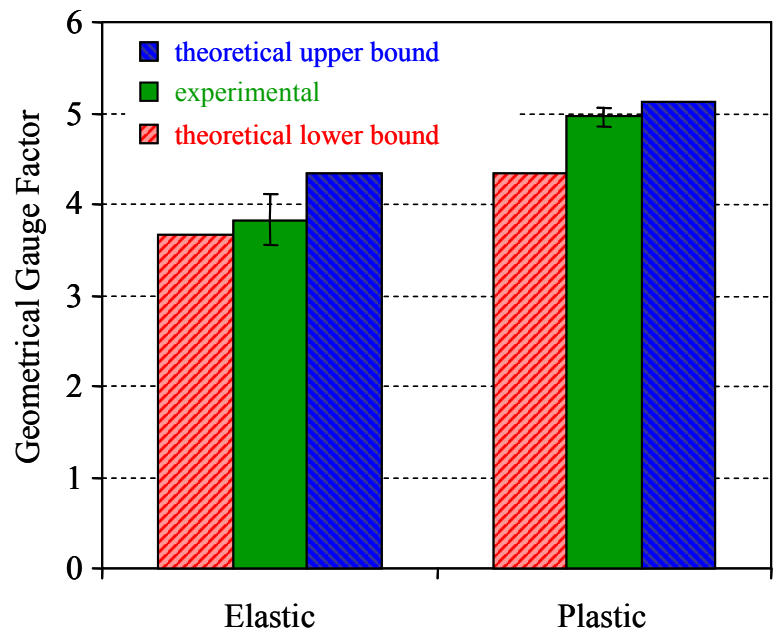

Fig. 25. Comparison between the experimental results on the geometrical gauge factor in both elastic and plastic ranges and their respective theoretical lower (thin plate) and upper (thick plate) bounds.

Next, we used non-contacting eddy current conductivity measurements to determine the material gauge factor independently from the usually much stronger geometrical effect. Figure 26(a) shows the results of elastic piezoresistivity measurement on 304 stainless steel. The reversible conductivity variation was measured using a Nortec $2000 \mathrm{~S}$ eddy current instrument with a racetrack-shaped eddy current probe coil of aspect ratio 4 at $300 \mathrm{kHz}$ using calibration blocks of $1.48 \%$ IACS and $2.53 \%$ IACS. By rotating this directional probe coil around its own axis, the apparent eddy current conductivity (AECC) was measured separately along the axial and lateral directions in the surface of the specimen. The axial and lateral piezoelectric coefficients were found to be $p_{11} \approx 1.35$ and $p_{12} \approx 1.95$, respectively (negative conductivity changes correspond to positive resistivity changes). Therefore, the differential piezoelectric coefficient is $p=p_{11}-p_{12} \approx-0.6$. For the sake of simplicity, this measurement was only done at room temperature since the elastic piezoresistivity is not expected to change much with temperature.

The irreversible electric anisotropy caused by plastic strain was measured using the same eddy current system at a frequency of $2 \mathrm{MHz}$. Figure 26(b) shows the average AECC results for one intact specimen and six others that were plastically strained to $15 \%$ at temperatures ranging from room temperature to $250{ }^{\circ} \mathrm{C}$. At room temperature, the AECC difference between the axial and lateral directions is approximately $A \approx 1.7 \%$. However, this difference gradually diminishes as the specimens are deformed at increasing temperatures and almost completely vanishes at $250{ }^{\circ} \mathrm{C}$ where the deformed specimen exhibits anisotropy $\approx 0.7 \%$ very similar to that of the intact material. The largest electric anisotropy $A \approx-1.7 \%$ caused by plastic strain $\varepsilon_{\mathrm{p}} \approx 15 \%$ at room temperature corresponds to a linearized plastic piezoresistivity coefficient of only $P=P_{12}-P_{12} \approx$ -0.1 . Since the directionality factor of a square-electrode PD probe is $D \approx 1.4-1.7$, even this level 
of deformation-induced anisotropy would cause only less than $0.2 \%$ change in the material factor $\xi_{\mathrm{m}} \approx 1+D A$ of the normalized resistance ratio, not to mention that the effect becomes weaker at elevated temperatures. Generally, material effects remain negligible until creep causes severe microscopic damage, such as aligned clusters of cavities and preferentially oriented microcracks, in the material.

a)

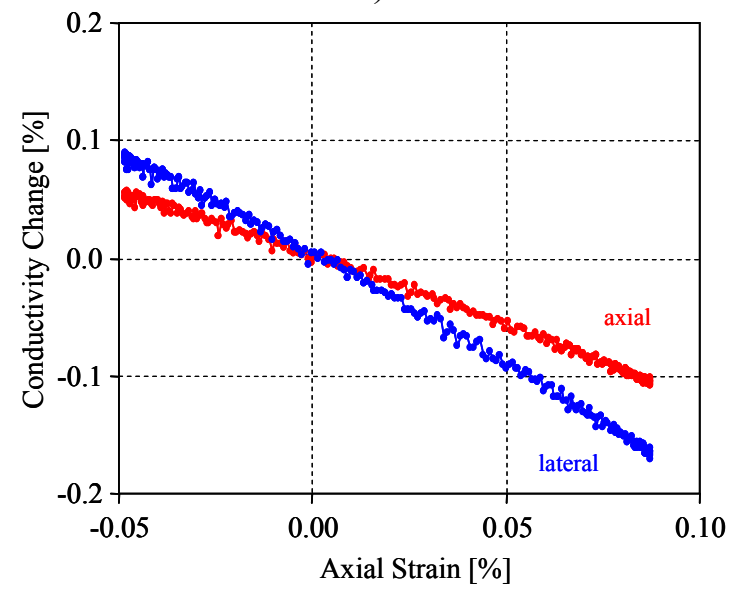

b)

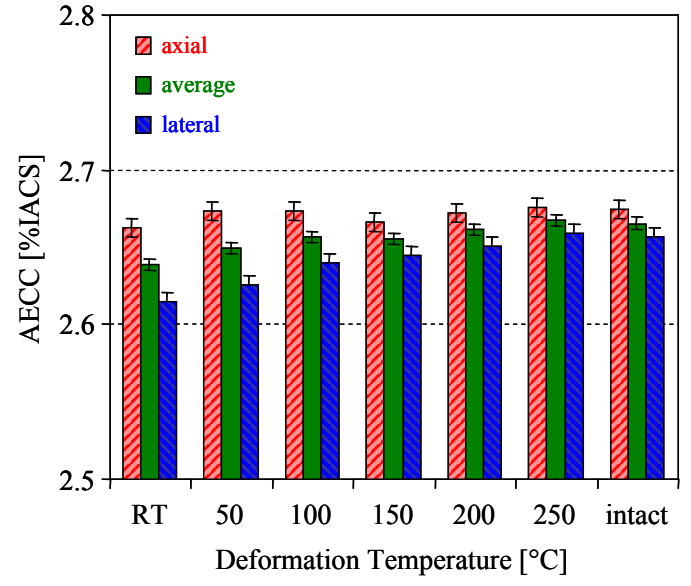

Fig. 26. (a) Elastic piezoresistivity coefficient measurement and (b) AECC variations in one intact and six plastically strained $(15 \%)$ specimens as the deformation temperature is increased in 304 stainless steel.

\subsection{Second generation DACPD system}

The first generation directional alternating current potential drop (DACPD) measurement system was used initially during our project to validate our analytical predictions and to establish the optimal measurement specifications for a dedicated instrument for creep monitoring. We determined that an ideal instrument would operate in a 4-wire AC resistance measurement mode and would allow multiplexing among 4 channels with independent polarity flipping to enhance the common mode rejection ratio (CMRR) of the system whenever necessary. The most important specifications of the creep monitoring system are listed in Table 2. A schematic block diagram and picture of the of the second generation experimental system developed for creep sensing by directional ACPD measurements is shown in Figure 27. This measurement system is based on an enhanced Stanford Research Systems SIM921 resistance bridge. The injection and sensing multiplexers play the same role as before. All other functions are integrated into the SIM921 resistance bridge.

We investigated the feasibility of increasing the signal-to-noise ratio (SNR) of our measurements with a parallel preamplifier configuration. We found that with ten-fold parallel processing of the ACPD signal the so-called flicker noise can be essentially eliminated even at very low frequencies and the theoretically possible lowest noise density limit can be achieved within $10-20 \%$. The achieved noise suppression not only allows us to collect data with less averaging in field tests, but also to monitor creep damage accumulation at earlier stages in laboratory tests. We have also developed an improved current injection method that not only increased the injected current level from $10 \mathrm{~mA}$ to $100 \mathrm{~mA}$, it also facilitated better common mode rejection ratio (CMRR) which translates into better suppression of measurement uncertainties caused by variations of the electrode contact resistances. 
Table 2 Specifications of the creep monitoring system.

\begin{tabular}{|c|c|c|c|c|}
\hline Parameter & Minimum & Typical & Maximum & Unit \\
\hline Measured Resistance & 1 & 10 & 50 & {$[\mu \Omega]$} \\
\hline Relative accuracy & \pm 0.5 & \pm 0.2 & & {$[\%]$} \\
\hline Temperature Stability & \pm 0.05 & \pm 0.02 & & {$\left[\% /{ }^{\circ} \mathrm{C}\right]$} \\
\hline Temperature range & 0 & & 80 & {$\left[{ }^{\circ} \mathrm{C}\right]$} \\
\hline Frequency & 1 & 3 & 30 & {$[\mathrm{~Hz}]$} \\
\hline Time Constant & 0.3 & & 3 & {$[\mathrm{~s}]$} \\
\hline Reading Rate & 1 & 2 & & [update/s] \\
\hline Resolution & & 1 & & {$[\mathrm{n} \Omega]$} \\
\hline Measurement Noise (RMS) & & 10 & 20 & {$\left[\mathrm{n} \Omega / \mathrm{Hz}^{1 / 2}\right]$} \\
\hline Lead Resistance & & & 10 & {$[\Omega]$} \\
\hline Input Impedance & 100 & & & {$[\mathrm{k} \Omega]$} \\
\hline Common Mode Rejection & 120 & 140 & & {$[\mathrm{~dB}]$} \\
\hline Excitation Current (RMS) & & 0.1 & 0.25 & {$[\mathrm{~A}]$} \\
\hline
\end{tabular}

One additional advantage of these developments is that we can increase the penetration depth of our ACPD measurements and thereby sample the electric properties of the material under test at larger depths below the surface, which requires not only increasing the electrode separation but also lowering the inspection frequency and thereby inevitably requires better noise suppression. Both of these new elements have been integrated into our ACPD system and have been tested successfully over extended time periods. The long-term and thermal stability, noise suppression, and CMRR of the upgraded system were all found to have greatly improved and in full compliance with the strict specifications listed in Table 2. 
a)

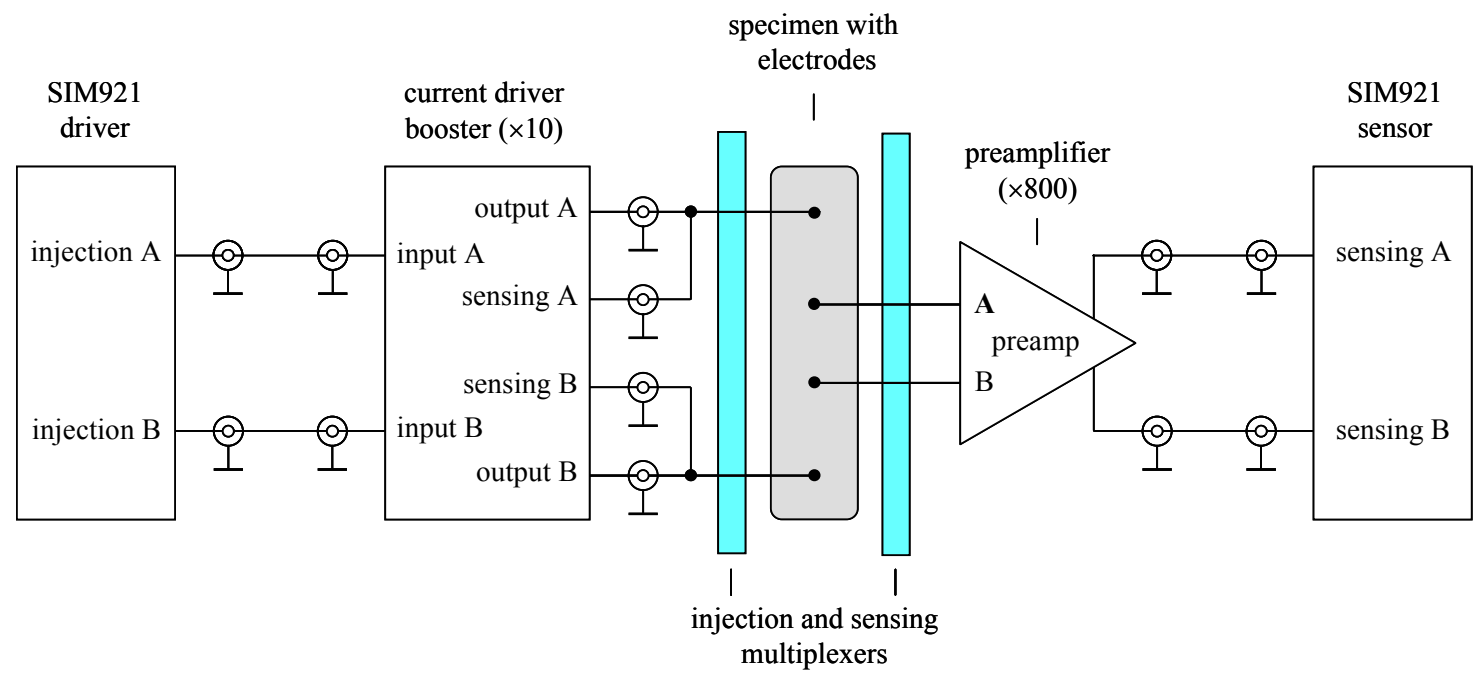

b)

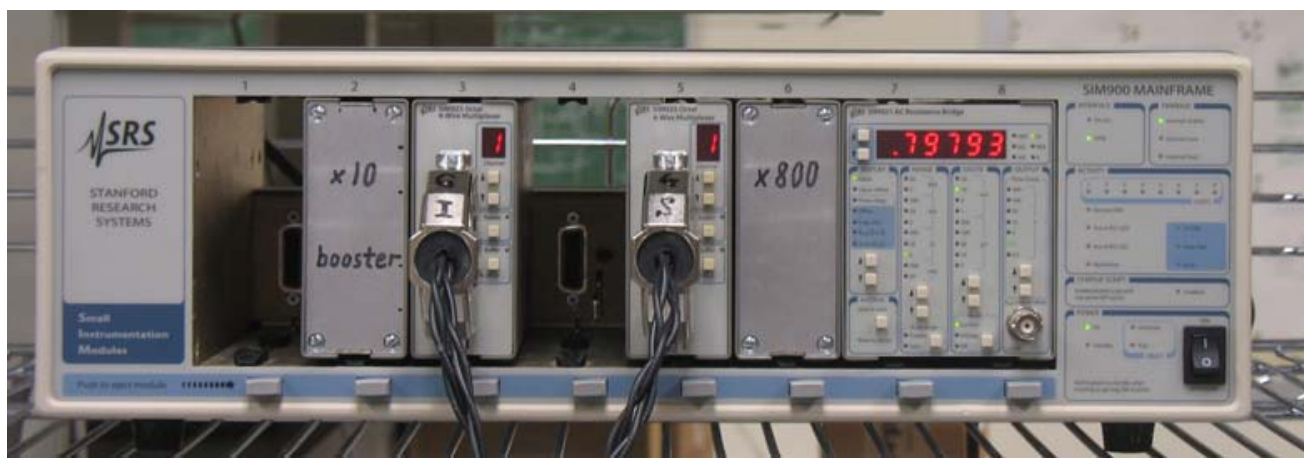

Fig. 27. The schematic block diagram (a) and picture (b) of the second generation ACPD system based on an enhanced SIM921 resistance bridge.

At the beginning of this project, we purchased a 2320-MM Lever Arm Tester from Applied Test Systems, Inc., for in-situ monitoring of creep. We received Grade 91 9Cr-1Mo material from Idaho National Laboratory (INL) and welded material from Utah State University. We produced creep specimens in a quantity that was sufficient for all our planned experiments under this project. Long-term robustness of the four-point electrode system requires that the spotwelded thermocouple wires be replaced by stud-welded electrodes of larger diameter. Earlier results indicated that when the diameter of the studs becomes larger than $20 \%$ of the separation between the electrodes, due to reversible and irreversible temperature changes of the electric conductivities of the Grade 91 steel to be monitored and the electrode material, their resistivity ratio might significantly change during the test. This relative conductivity change might cause migration of the effective injection and sensing points relative to the centers of the electrodes, which in turn causes a small change in the monitored NDE parameter irrespective of actual creep. We have conducting both Comsol Multiphysics Finite Element simulation and experimental tests of this issue and found that with careful design the effect can be kept at acceptable levels. This problem was avoided in our laboratory tests by using spot welded 
thermocouple wire electrodes as it was previously shown in Figure 20(a). The wire electrodes were positioned with a ceramic template that was either removed by breaking it or left in place during the creep test as it is shown in Figure 28.

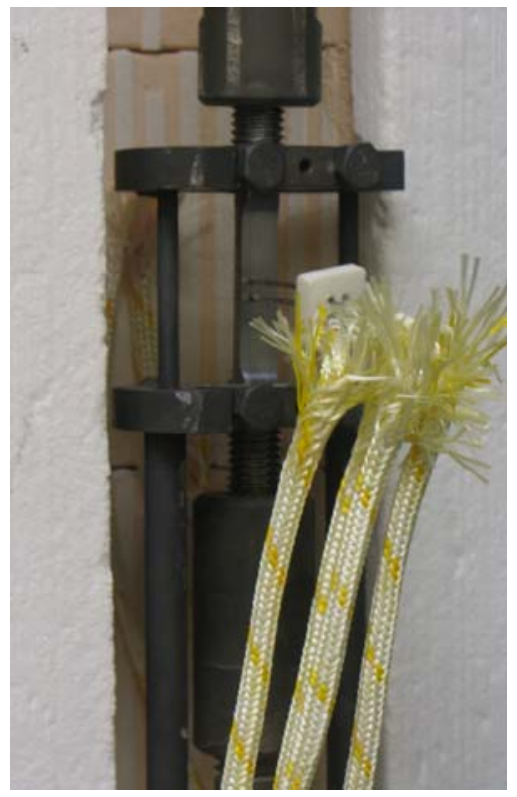

Fig. 28. Spot welded thermocouple wire electrodes and positioning ceramic template with a creep specimen mounted inside the furnace of the 2320-MM Lever Arm Tester.

\subsection{Creep tests with DACPD monitoring}

Creep tests were conducted on Grade 91 9Cr-1Mo material between 70 and $150 \mathrm{MPa}$ stress levels at $650{ }^{\circ} \mathrm{C}$. As an example, Figure 29 shows typical creep monitoring test results taken at $120 \mathrm{MPa}$ stress level. First, the specimen was monitored for 24 hours at room temperature and then heated to $650{ }^{\circ} \mathrm{C}$ and kept there at constant temperature for the rest of the test as shown in Figure 29(a). The specimen was "soaked" at $650^{\circ} \mathrm{C}$ for 10 hours before it was loaded. The axial and radial resistances were measured at every 4 minutes. Both resistances were normalized to their respective initial values to eliminate minor differences caused by electrode positioning. Figure 26(b) shows these normalized resistances $\eta_{1}$ and $\eta_{2}$ as well as their average $\eta_{\text {avg. Both }}$ resistances increase by more than $100 \%$ upon heating, but their ratio remains essentially constant. As expected under uniaxial stress conditions, $\eta_{1}$ increases while $\eta_{2}$ decreases during creep, while $\eta_{\text {avg }}$ increases just a little bit since the axial tensile strain is twice as high as the lateral compressive strain. Figure 29(c) shows the NDE strain $\varepsilon_{\mathrm{NDE}}$ calculated from the normalized resistance ratio $\xi=\eta_{1} / \eta_{2}$ using the nonlinear inversion equation of Eq. (44) as well as the average strain over the gauge section of the specimen calculated from the overall elongation of the specimen measured by a high-temperature extensometer. Generally, the comparison between the NDE strain and the extensometer result is very good up to the end of the secondary creep regime. At the beginning of the tertiary creep two things happen. First, directional creep damage forms, which increases the apparent NDE strain because of the added effect of directional material damage. Second, necking initiates at the location of the future rupture, 
which leads to a local strain that is either higher or lower than the average strain depending on whether the monitored spot is inside or outside the necking area (in this case it was outside).

a)

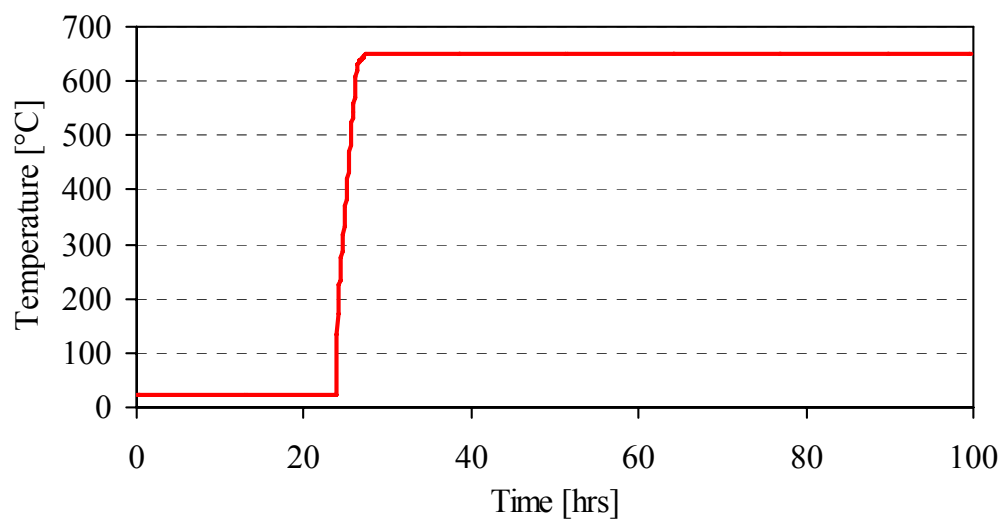

b)

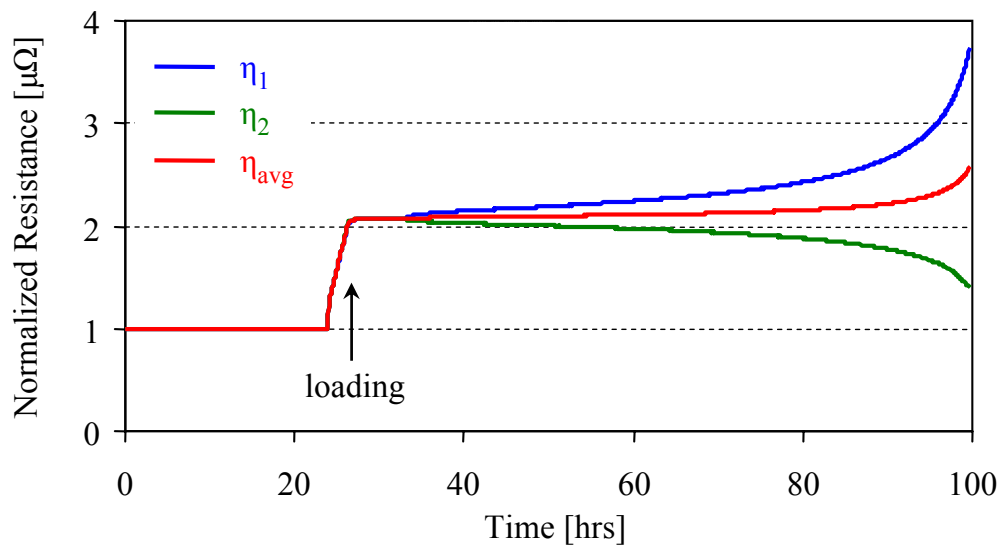

c)

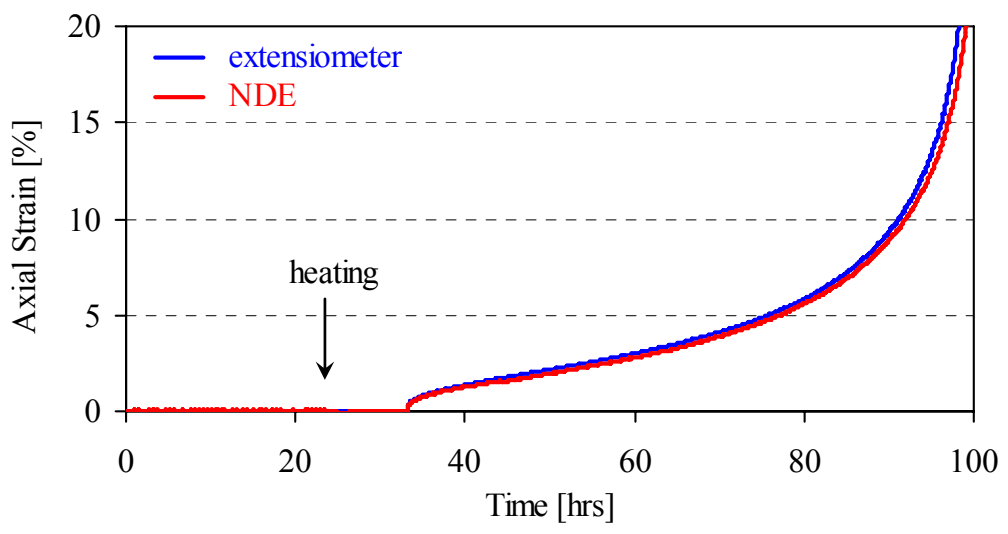

Fig. 29. Creep monitoring test results in a Grade 91 9Cr-1Mo material at $120 \mathrm{MPa}$ stress level at $650{ }^{\circ} \mathrm{C}$. 
In some cases, a small spike can be observed in the NDE creep strain during fast temperature gradients. The reason for those artifacts is that the axial and lateral resistances are measured sequentially rather than simultaneously. During sharp temperature variations, the small reversible resistivity difference caused by the slightly different temperatures at which the two compared resistances are measured might cause a temporary discrepancy that appears as a small spike in the inverted NDE strain. These artifacts can be minimized by comparing the axial resistance to the average of the lateral resistances taken before and after the axial measurement. However, even this apparent synchronization of the axial and lateral measurements might leave a remnant difference during very fast temperature transitions, which then should be removed by additional artifact filtering.

Figure 30 shows the inverted NDE strain results in Grade $919 \mathrm{Cr}-1 \mathrm{Mo}$ material between 70 and $150 \mathrm{MPa}$ stress levels at $650^{\circ} \mathrm{C}$. The $140 \mathrm{MPa}$ test was repeated twice, the $125 \mathrm{MPa}$ test five times, and the $100 \mathrm{MPa}$ test twice to study the reproducibility of the creep curves. It can be seen that the results are reproducible within the natural uncertainty of creep tests. The shortest test we conducted lasted a little less than 5 hours at $150 \mathrm{MPa}$ while the longest test took more than 3,600 hours or 150 days at $70 \mathrm{MPa}$.

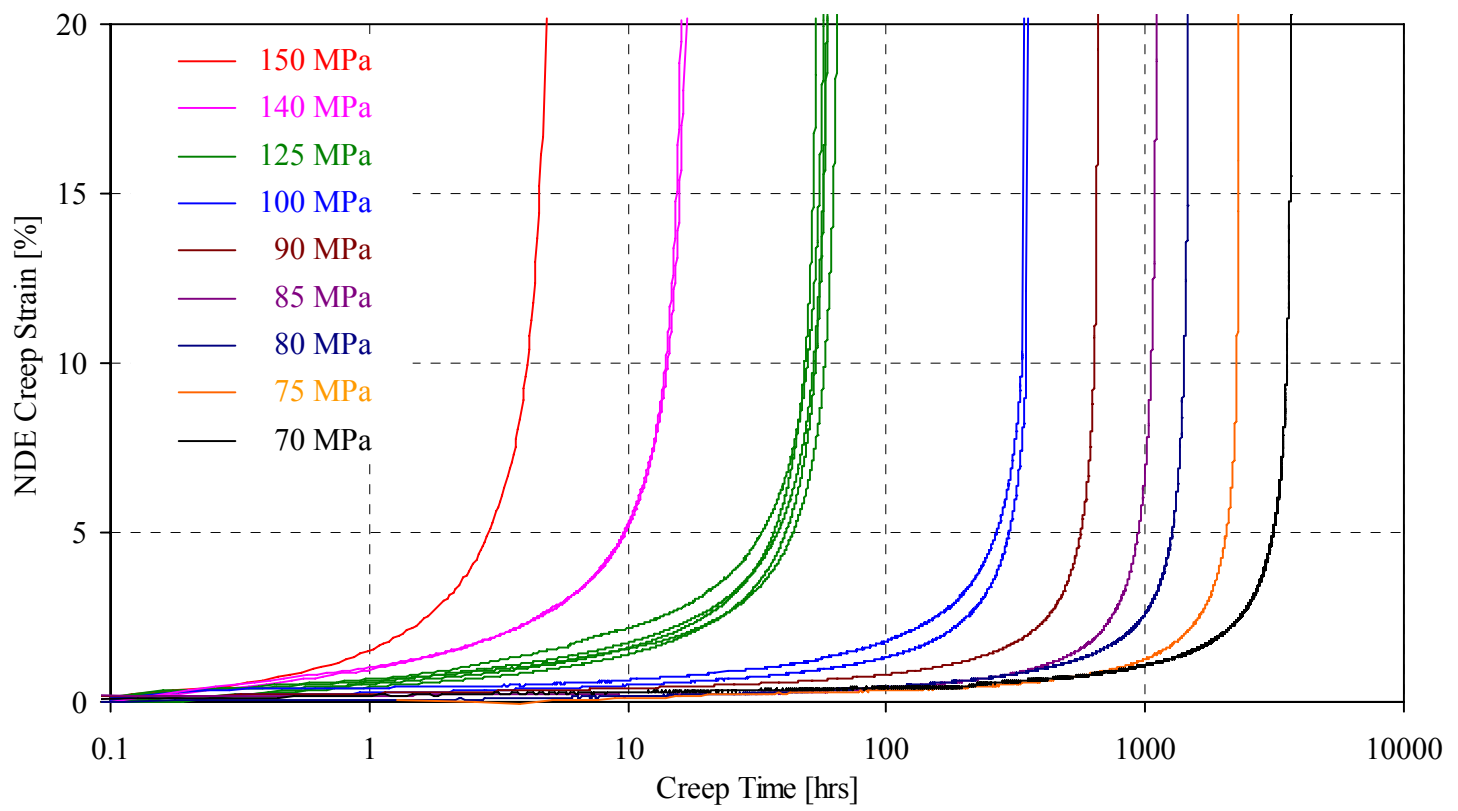

Fig. 30. Inverted NDE strain results in Grade 91 9Cr-1Mo material between 70 and 150 MPa stress levels at $650^{\circ} \mathrm{C}$.

Generally, the feasibility of any NDE technique is dependent on the presence of perceivable precursors well before failure so that they can be detected by inspections during regular maintenance periods. The working hypothesis of our project was that this conventional mode of operation of NDE cannot be adapted to creep damage detection in 9Cr-1Mo steel because perceivable damage forms only too close to rupture. Therefore, we adopted a structural health monitoring (SHM) approach that avoids this problem by essentially continuously monitoring the state of the material at critical locations. The feasibility of this approach cannot be fully established by using highly accelerated tests only since the fraction of creep life when the material exhibits perceivable creep failure precursors dramatically changes with increasing creep 
rate. Therefore, we ran creep monitoring tests at as low creep rates as it was possible within the time limitation of our project. At the same time, it became obvious from our tests that as the stress level decreased and the creep life increased, more an more of the total creep life was spent at low plastic strain levels. Figure 31 shows the relative percentage of the total creep life spent below given plastic strain levels, namely $2 \%, 4 \%$ and $8 \%$, as a function of stress in Grade 91 $9 \mathrm{Cr}-1 \mathrm{Mo}$ material at $650{ }^{\circ} \mathrm{C}$. This figure was constructed by analyzing the fifteen creep curves plotted in Figure 30. At each stress level rupture occurred at strain levels in excess of 25-30\%. At $150 \mathrm{MPa}$, the material spent only $18 \%$ of its roughly 5 hours of creep life below $2 \%$ of plastic strain. In comparison, at a much lower stress level of $70 \mathrm{MPa}$, the material spent close to $60 \%$ of its more than 3,600 hours of creep life below the same $2 \%$ plastic strain threshold. In relative terms, the onset of tertiary creep with high strain rate represents less of the total creep life of the material when the stress level is lower and therefore the creep life is longer.

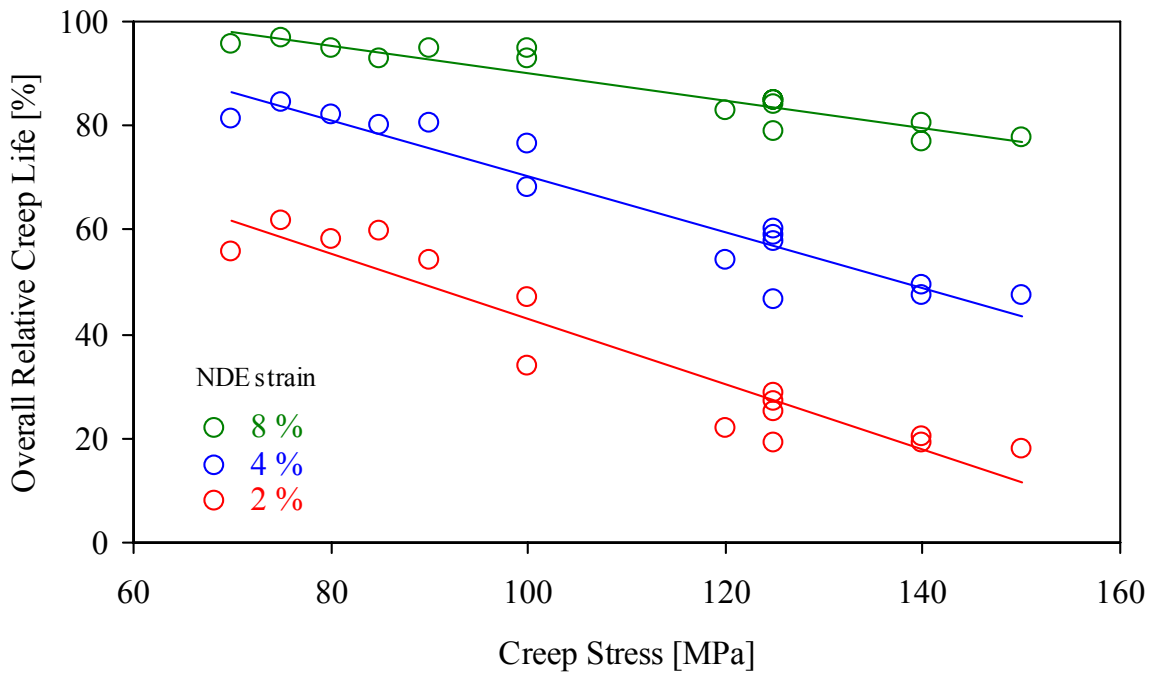

Fig. 31. Relative percentage of the total creep life spent below given plastic strain levels in Grade $919 \mathrm{Cr}-1 \mathrm{Mo}$ steel during creep at $650{ }^{\circ} \mathrm{C}$ and various stress levels.

In addition to the above described full-length creep tests conducted up to the ultimate rupture of the specimens, we also conducted partial-life tests with DACPD monitoring to assess the associated microstructural evolution and damage initiation and accumulation in Grade 91 9Cr1Mo material. Figure 32 shows the NDE strain inverted from DACPD measurements on four specimens crept to rupture, two crept to $30 \%$ of average rupture time, and one crept to $60 \%$ of average rupture time at $120 \mathrm{MPa}$ stress level and $650{ }^{\circ} \mathrm{C}$. At this combination of stress and temperature levels the average creep life was found to be 53.6 hours, so the partial creep tests were stopped after 16 hours (30\%) and 32 hours (60\%). Naturally, the partial creep curves are well within the expected variation of the data based on the full creep tests. These specimens then were evaluated for the presence of creep damage by metallurgical means. The results of these metallurgical tests are presented in the next chapter. 


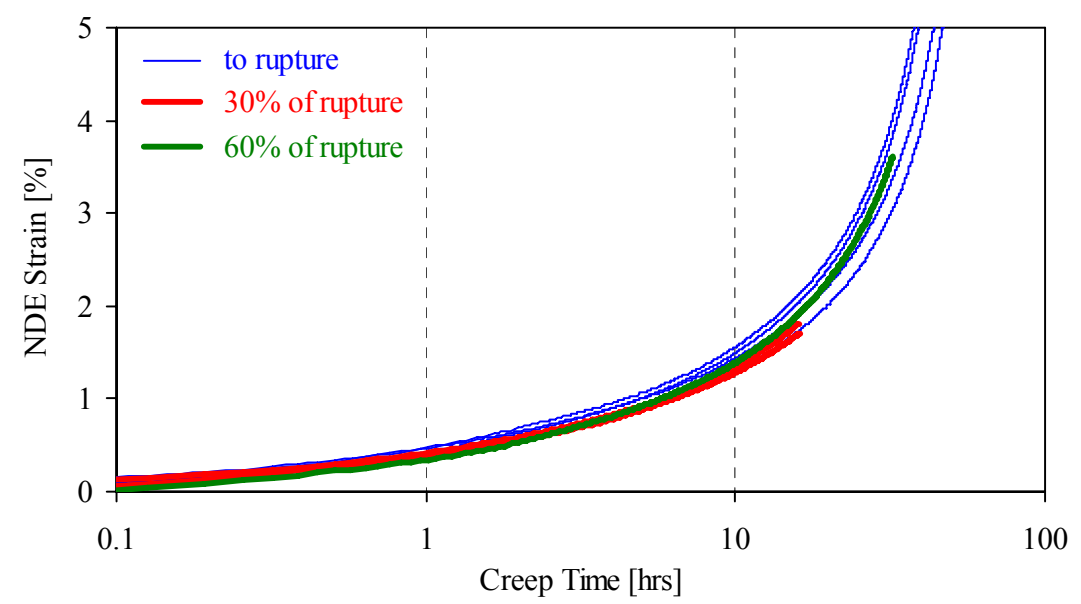

Fig. 32. NDE strain inverted from DACPD measurements on four specimens crept to rupture, two crept to $30 \%$ of average rupture time, and one to $60 \%$ of average rupture time at $120 \mathrm{MPa}$ stress level and $650{ }^{\circ} \mathrm{C}$.

\subsection{Creep tests in welds}

It is well known that creep damage tends to concentrate in welds and, especially, at the boundary between the weld and the heat-affected zone (HAZ) of the base metal. The applicability of DACPD creep monitoring in that transitional zone raises some technical problems since the weld is often much less conductive than the base metal itself. Tests were conducted to study the effect of thermal exposure on the average resistance and intrinsic anisotropy of the weld and base metals separately. In more or less homogeneous materials, i.e., far away from weld/base metal interfaces, the only issue is whether the intrinsic electric anisotropy of the material might change due to thermal exposure, but this effect is not expected to be significant. However, the apparent anisotropy measured in the vicinity of weld/base metal boundaries could exhibit much more significant changes because the resistivity of the weld and base metals could change at different rates during thermal exposure, which influences their resistance ratio and therefore the level of apparent anisotropy for a given probe position, especially if the probe straddles the boundary, but is not centered directly over it.

We have prepared five cross-weld samples for creep testing from a large piece of welded pipe provided by Professor Li of Utah State University. The "apparent" creep caused by different rates of thermally-activated microstructural evolution in the weld and base metal was small but detectable. Thermal exposure tests between $650^{\circ} \mathrm{C}$ and $750{ }^{\circ} \mathrm{C}$ over periods up to 300 hours showed that the intrinsic anisotropy of both the weld metal and the base metal remained essentially constant at $1 \%$. The average resistance for both the base metal and weld exhibited some increase during thermal exposure. However, in the weld metal the changes were barely noticeable while larger changes occurred in the base metal where the resistance increased as much as $6-7 \%$ at the end of the 300 -hour exposure at $750{ }^{\circ} \mathrm{C}$. This means that high-temperature thermal exposure will reduce the resistivity difference between the weld and the base metals, at least at peak temperatures, and cause weak apparent creep indications. In the case of deployable electrodes, both experimental and theoretical studies showed that the relative position of the probe to the boundary line can be optimized to suppress the effect. Alternatively, increasing the electrode separation can be used to suppress this effect. 
In the case of permanently mounted electrodes this is a minor effect compared to the expected 20-30\% maximum creep strain. Five welded specimens were tested to rupture at 120 $\mathrm{MPa}$ and $650{ }^{\circ} \mathrm{C}$. Figure 33 shows the average creep strain as measured by a high-temperature extensometer in these five welded G91 specimens. One specimen (\#1) had its gauge section machined in the base metal and it failed after 57 hours, which is perfectly consistent with the rupture life of numerous specimens machined from the intact G91 material provided by the Idaho National Laboratory. The other four specimens were machined so that their gauge section included the weld (\#2, \#3, and \#4) and the interface between the weld and the base metal (\#5). These specimens failed 30-60\% earlier than the Grade 91 base metal did under the same creep conditions. This is a very large scatter that is caused by the significantly lower total creep life of welded specimens and the larger variability of failure modes in welded samples. The average creep strain measured by the extensometer reached maxima between 5 and $11 \%$, well below the $25-35 \%$ typically seen in similar tests conducted on the intact G91 base metal. This indicates that whenever failure occurs in either the weld or at the interface between the weld and the base metal, tertiary creep is highly concentrated at the future failure site, therefore the average creep over the gauge section remains modest.

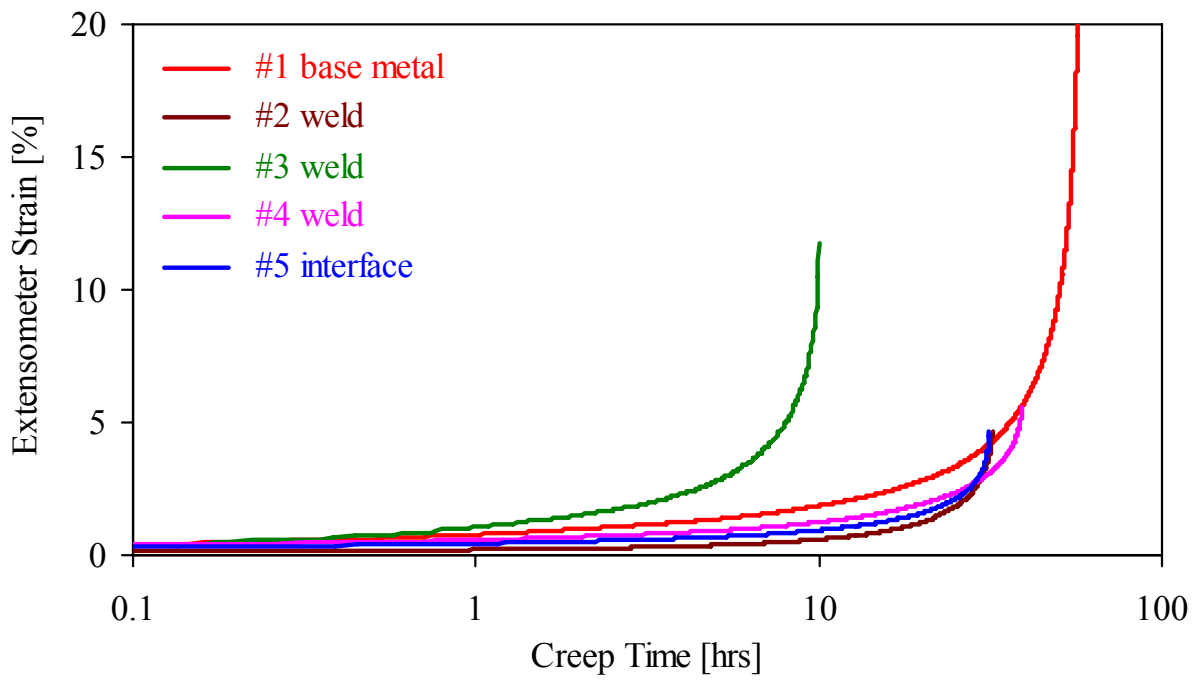

Fig. 33. Average creep strain as measured by a high-temperature extensometer in five welded G91 specimens crept to rupture at $120 \mathrm{MPa}$ stress level and $650{ }^{\circ} \mathrm{C}$ temperature.

As an example, Figure 34 shows the images of specimen $\# 5$ before and after testing. Before creep testing the interface between the weld and the base metal has been accurately located using a small-diameter eddy current pencil probe and the DACPD probe was spot welded to the surface over this transition region. Compared to specimens machined from the intact base metal, this specimen showed very little overall necking indicating a highly concentrated failure mechanism. 
a)

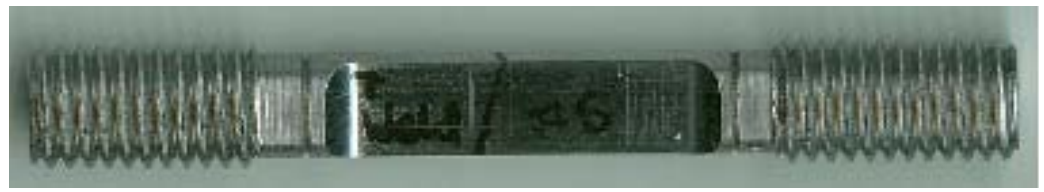

b)

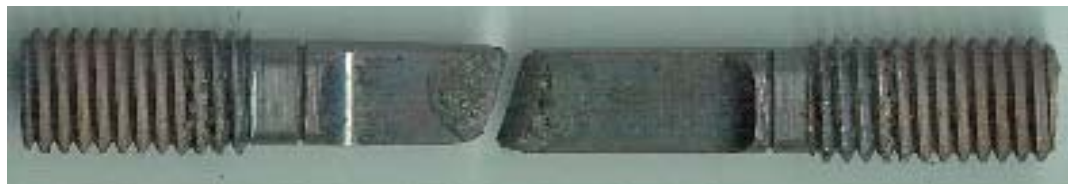

Fig. 34. Images of specimen \#5 before and after testing. The specimen failed in the monitored interface region.

Figure 35 shows the NDE strain obtained by inverting the DACPD data in the same five welded G91 specimens. Comparison to the average strain results obtained by the hightemperature extensometer reveal that in some cases (\#3 and \#5) the local strain measured by the DACPD probe is much higher while in other cases (\#2 and \#4) it is much lower. The reason for this is that, in the case of highly uneven tertiary creep distribution, the local NDE strain can be either higher or lower than the global average strain. As it was shown in Figure 34, the DACPD measurement was conducted essentially over the future rupture zone, therefore the local NDE strain was much higher than the global average strain.

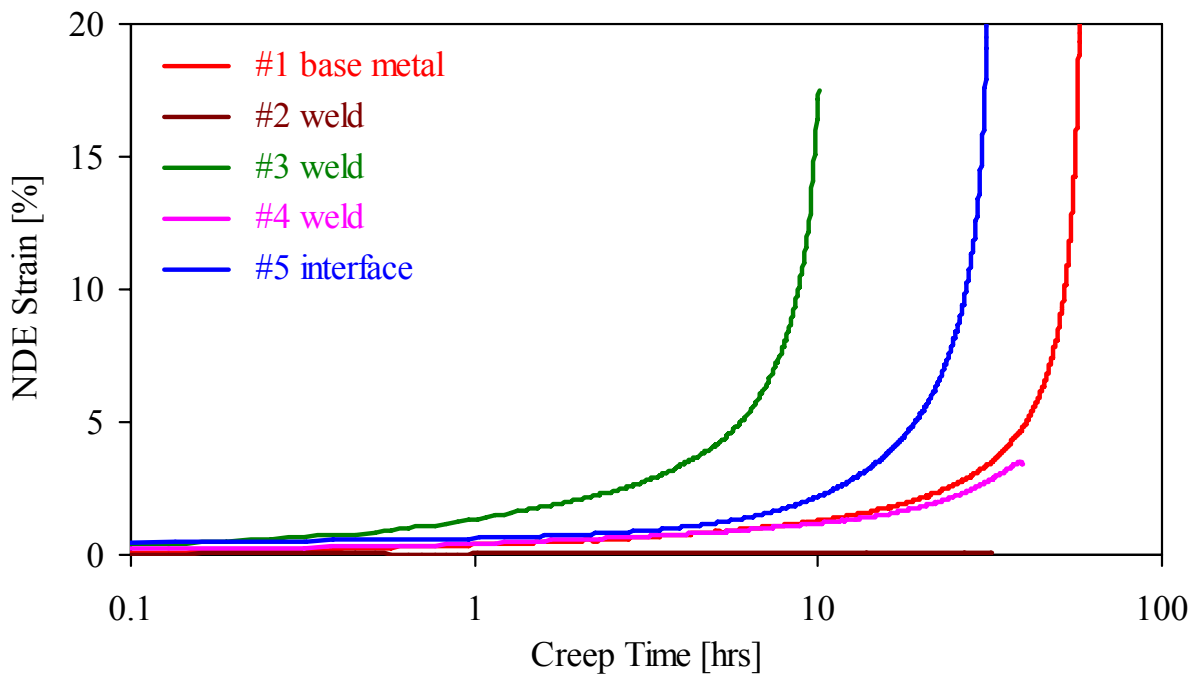

Fig. 35. NDE strain obtained by inverting the DACPD data in the same five welded G91 specimens crept to rupture at $120 \mathrm{MPa}$ stress level and $650^{\circ} \mathrm{C}$ temperature.

As an example, Figure 36 shows the images of specimens \#2 and \#4 after testing. Before creep testing the interface between the weld and the base metal has been accurately located using a small-diameter eddy current pencil probe and the DACPD probe was spot welded to the surface over this transition region. Again, compared to specimens machined from the intact base 
metal, these specimens showed very little overall necking indicating a highly concentrated failure mechanism.

a)

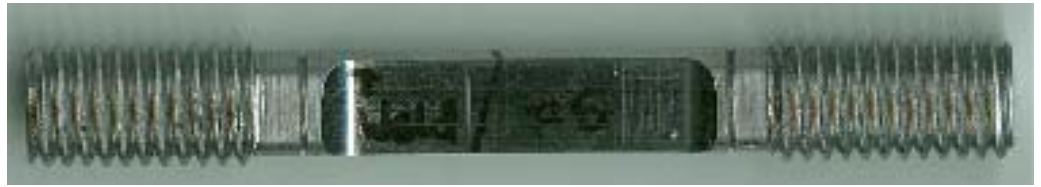

b)

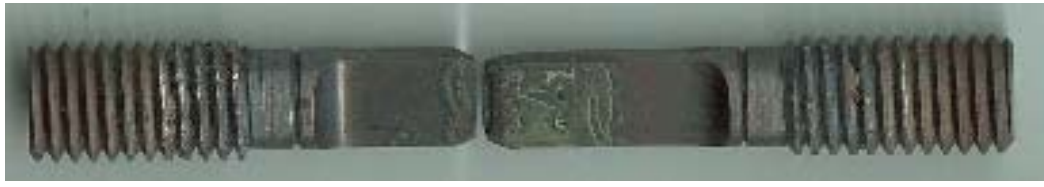

Fig. 36. Images of specimens \#2 and \#4 after testing. Both specimen failed outside the monitored region.

It is interesting to compare the local NDE strain measured by the DACPD probe and the global average strain measured by the extensometer. Figure 37 shows such a comparison of specimen \#1 that failed in the base metal, specimen \#3 that failed in the weld, and specimen \#5 that failed at the weld/base metal interface. In the case of the base metal, the tertiary creep occurs all over the gauge section therefore the global average strain closely follows the local NDE strain. In the case of failure inside the welded material, the tertiary creep is more concentrated at the future rupture site therefore the global average strain significantly underestimates the local NDE strain. Finally, in the case of failure at the critical interface between the weld and the base metal, the tertiary creep is very highly concentrated at this boundary therefore the global average strain very significantly underestimates the local NDE strain almost up to the ultimate rupture.

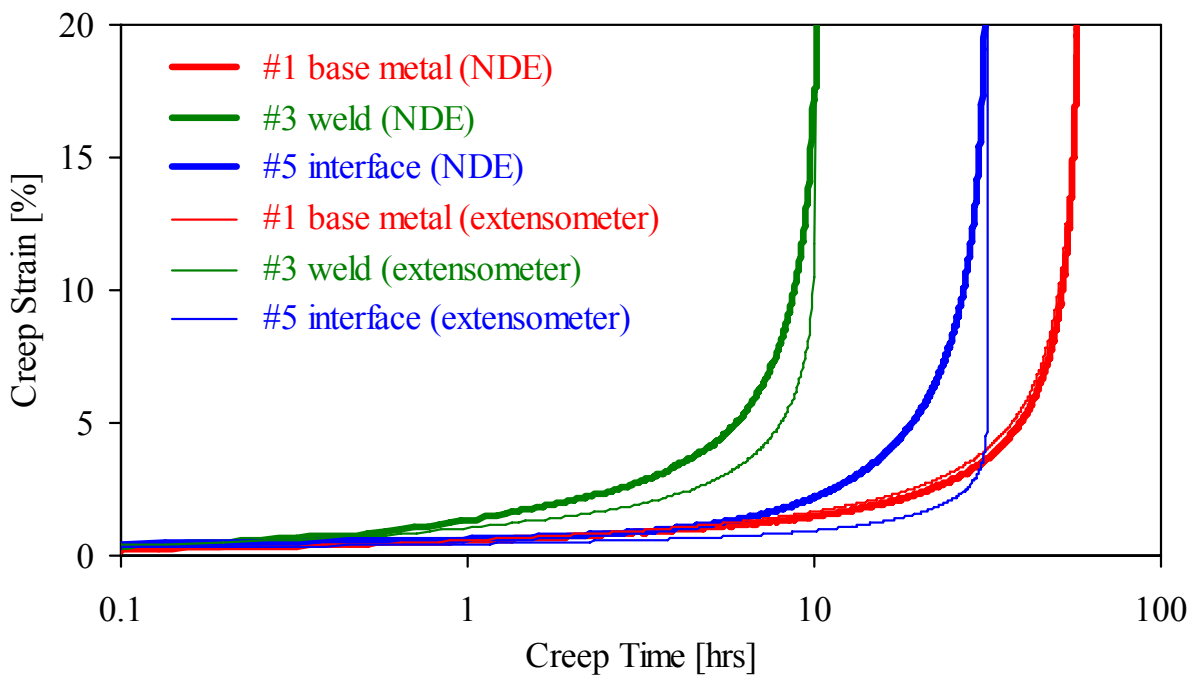

Fig. 37. Local NDE and global average strains in three welded G91 specimens crept to rupture at $120 \mathrm{MPa}$ stress level and $650{ }^{\circ} \mathrm{C}$ temperature. 


\subsection{Creep tests in Gleeble thermo-mechanical simulator at INL}

As part of this project, we also attempted to transition the DACPD creep monitoring technology to our DoE laboratory partner, the Idaho National Laboratory (INL). This effort focused on conducting similar tests described in earlier parts of this report in the Gleeble thermo-mechanical simulator of INL. This is a general purpose materials testing system with the capability of applying tensile or compressive loads to a sample while heating. This system is also capable of conducting testing in a vacuum, and in an inert environment as desired. The equipment is capable of heating rates as high as $10,000{ }^{\circ} \mathrm{C} / \mathrm{s}$ as the sample is electrically heated during operation. Temperature is measured by means of a thermocouple attached to the sample, and energy input is regulated via a large silicon controlled rectifier (SCR). The regulation of temperature in the Gleeble results in switching harmonics in the sample, so although the heating frequency is well above the sample testing frequency, the signal has substantial sub-harmonics in the range of the ACPD testing frequency. This results in noise far stronger than usual levels encountered in furnaces of creep machines that overwhelms the low-frequency ACPD signal, making it impossible to collect data while the Gleeble's thermal control is active. In an effort to enable testing, the control software originally provided by researchers at the University of Cincinnati (UC) was rewritten to enable samples to be collected on-demand, and enable the acquisition of a number of signals that should be averaged for a single data point. With these modifications the software developed at UC collected only the non-inverted "forward" resistivity in the axial and tangential directions so only two samples needed to be taken at each test point. This reduced the total measurement time per sample to roughly 45-50 seconds.

Figure 38 shows the sample prepared with signal and sense leads spaced $2 \mathrm{~mm}$ apart on one side, and a thermocouple on the edge for temperature control. Data was collected on two occasions; the first set of data was collected during operation of the Gleeble without any heating of the sample. The first sample was pulled to failure at room temperature, and data was collected at several stress points. Figure 39 shows how the normalized axial and lateral resistances changed as a function of tensile stress at room temperature. These results clearly indicate plastic strain when the sample yields.

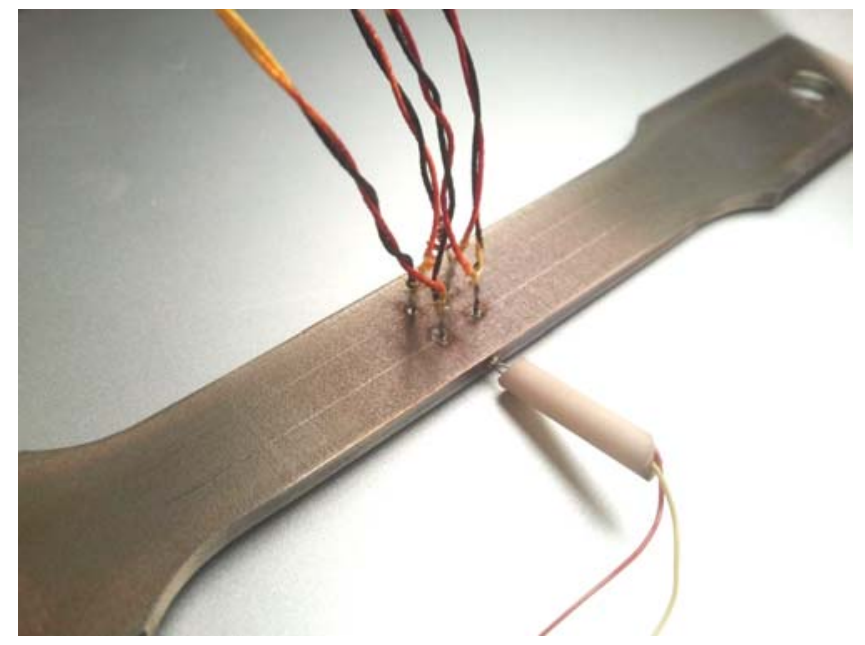

Fig. 38. Sample prepared with signal and sense leads spaced $2 \mathrm{~mm}$ apart on a side, and a thermocouple on the edge for temperature control. 


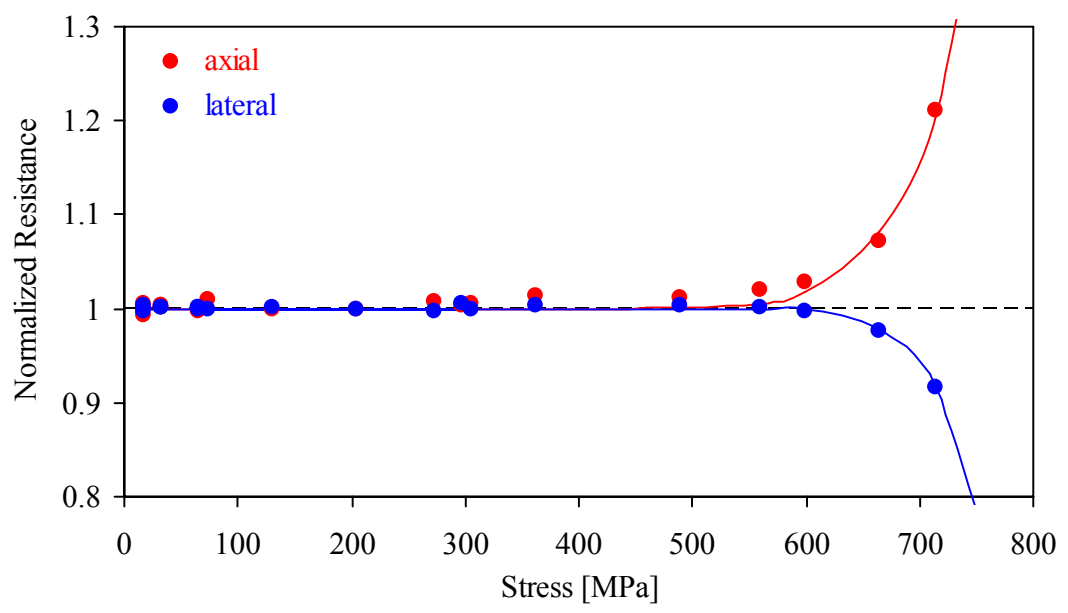

Fig. 39. Normalized axial and lateral resistances as functions of tensile stress at room temperature in G91 steel.

In a second test, the sample was mounted in the Gleeble system, pulled at successively higher loads, and held at $300{ }^{\circ} \mathrm{C}$. Figure 40 shows the results of this test in a Grade 91 9Cr-1Mo specimen. Figures 40(a) and 40(b) show the temperature and loading profiles. To enable the collection of sample data, the heating was turned off during sample collection, as the sample cools, and as a result data was collected at lower temperatures. For the lateral direction the data was collected at roughly $250^{\circ} \mathrm{C}$, and for the axial direction the sample had cooled to roughly $200{ }^{\circ} \mathrm{C}$. The temperature was recorded at each data collection point during testing. The substantial temperature difference between the axial and lateral measurements perceivably compromises the thermal common mode rejection of the system. Figure 40(c) shows the normalized resistance ratio as a function of time during the test.

In this part of the project, INL was successful in replicating the testing method developed at UC with the prototype DACPD equipment installed with help from UC. These tests demonstrated that the heating of a sample via electrical conduction can result in untenable signal to noise ratios, and further testing at the INL should be conducted in conventional load frames, or if testing is to be conducted in the Gleeble system, an alternate means of heating the sample, such as a clam-shell heater, should be developed. INL has also developed a version of the software that allows on-demand sampling via this method. This software can aid in future development and research of this method. 
a)

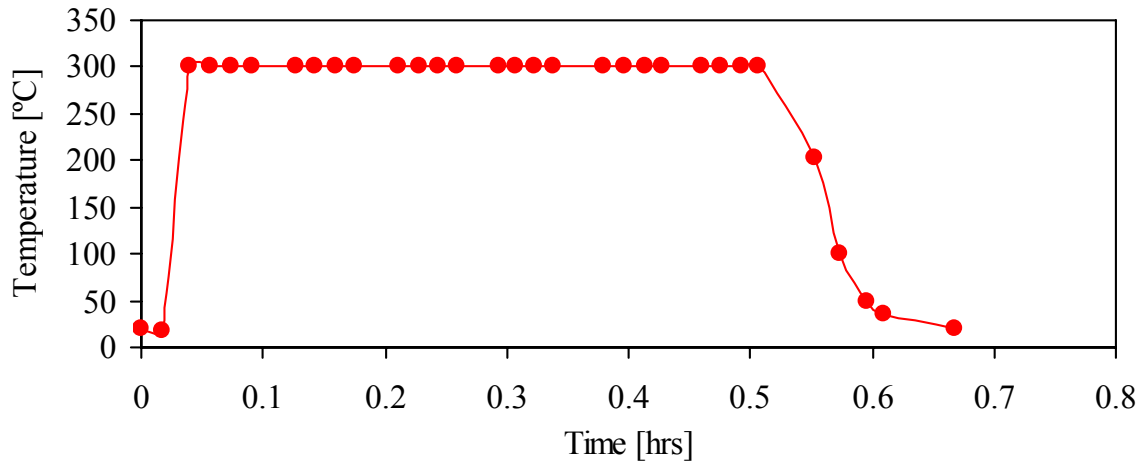

b)

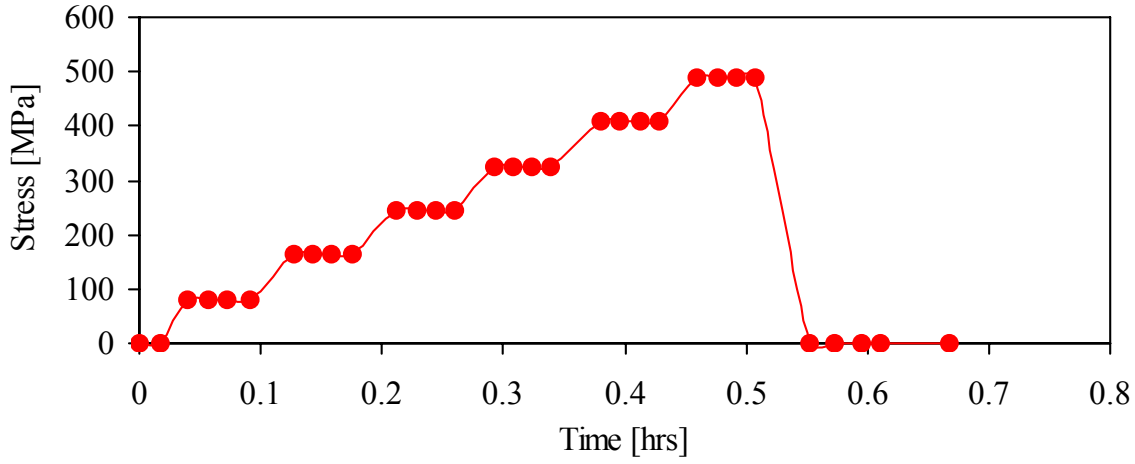

c)

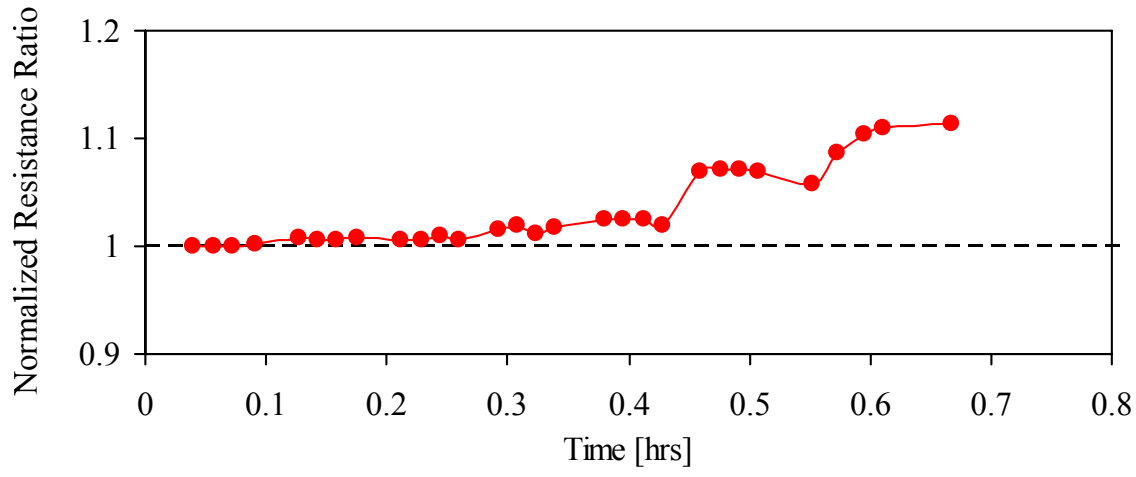

Fig. 40. Creep monitoring test results in a Grade $919 \mathrm{Cr}-1 \mathrm{Mo}$ specimen $300{ }^{\circ} \mathrm{C}$.

\section{Metallurgical Investigation}

This effort was aimed at microstructural characterization of the samples in heat treated and creep tested conditions. The results of microstructural characterization supplement the previously presented NDE results and make it possible to establish the relationships between the two and the physical, microstructural and mechanistic basis for understanding the changes in the properties.

Modified 9Cr-1Mo steels also called as G91 steels have widespread applications in nuclear power plants as boiler tubes, steam pipes and reactor pressure vessels. In future, for boiler 
constructions G91 has an advantage over 300 series stainless steels in USA [52 ]. Kleuh [53] has discussed various alloys developed in the past used in core and out of core for the GEN IV nuclear plants and qualified F-M $(9-12 \% \mathrm{Cr})$ steels to have a good standing in the list because of high corrosion resistance and thermal stability at high temperatures. F-M steels are also known for high ductility after irradiation and reduced activity (RA) property [54]. The microstructure of these F-M steels consist of sub-grain boundaries decorated with $\mathrm{Cr}$ rich $\mathrm{C}_{23} \mathrm{C}_{6}$ and matrix dotted with $\mathrm{MX}(\mathrm{X}=\mathrm{V}, \mathrm{Nb})$ precipitates which gives it high-temperature strength [55,56]. Microstructural stability of Modified $9 \mathrm{Cr}-1 \mathrm{Mo}$ alloy at high temperatures up to $650{ }^{\circ} \mathrm{C}$ under static tensile and creep conditions was reported by Jones et al. [57]. Structural properties of Mod. 9Cr-1Mo steels have been extensively studied in the past two decades. Sonderegger et al. [58] reported that creep in these F-M alloys proceeds by sub-grain coarsening, but the grains do not coarsen. Maruyama et al. [59] studied the long-term creep behavior of G91 steels. They suggested that sub-grain boundaries are stabilized by carbides and static recovery of sub-grains leads to creep deformation. Recovery of dislocations is also a part of creep deformation. These carbides become unstable at high temperatures and aggregation of carbides occurs. Hence the pinning force of the carbides is decreased and coarsening of sub-grains starts to happen as creep proceeds. Anderson et al. [60] suggested that the carbides along the grain boundaries are $\mathrm{M}_{23} \mathrm{C}_{6}$. As the creep proceeds in G91 steels the MC and MX carbides inside the grain dissolve and the carbides along grain boundaries grow at the expense of these carbides. On the other hand, Cerri et al. [61] reported the bimodal carbide distribution and coarsening of both types of carbides during creep.

It can be concluded that long term creep in G91 steels proceeds by aggregation of carbides and sub-grain recovery. The damage evolution during creep can vary at high strain rates and high stress levels. Most reported works were conducted in the domain of long-term creep at low stress levels and less emphasis has been given to microstructural evolution during short-term creep at high stresses. It should be noted that the operating characteristics of GEN IV high temperature structural components involve long service lives ( $>60$ years). As it is not often practical to perform long-term creep tests under these conditions and obtaining creep-fatigue test data required to determine negligible creep condition is very challenging because of the long test times that are involved, it is a prudent strategy to compensate for the high uncertainty in the negligible creep conditions by implementing NDE procedures to achieve better health/safety management of nuclear power plants. We investigated the feasibility of a novel yet simple NDE method that is capable of reliable, in-situ monitoring of creep damage and changes in the microstructure of materials and components in the field.

The aim of the present study was to evaluate the creep behavior, damage evolution and microstructural changes during short-term creep at different stress levels varying from $80 \mathrm{MPa}$ to $140 \mathrm{MPa}$ and link the microstructural changes to the NDE results for reliable prediction of microstructure and damage evolution. Different creep time intervals at a temperature of $650{ }^{\circ} \mathrm{C}$ have been developed and various parameters including cavity nucleation, carbide evolution and sub-grain development as well as microstructure and grain size were investigated in detail. Efforts have been made to better understand the degradation mechanisms of this alloy at higher stress levels.

\subsection{Inspection methods}

Two blocks of the modified 9Cr-1Mo alloy were obtained from Idaho National Lab. The blocks had been given the heat treatment recommended for Mod. 9Cr-1Mo steels for producing an initial microstructure of tempered martensite. 


\section{Creep Testing}

Creep tests were carried out on the samples at a fixed temperature of $650{ }^{\circ} \mathrm{C}$ and at different stress levels, namely, $140 \mathrm{MPa}, 120 \mathrm{MPa}, 100 \mathrm{MPa}$, and $80 \mathrm{MPa}$. Creep tests were also carried out at different time intervals at the stress values of $120 \mathrm{MPa}, 100 \mathrm{MPa}$, and $80 \mathrm{MPa}$. The time intervals included $25 \%$ life, $50 \%$ life and $75 \%$ life. The total fracture life at a given stress level was initially established by performing 3 or 4 creep tests up to failure and the total time taken up to fracture was averaged. Then the $25 \%, 50 \%$, and $75 \%$ creep life values were calculated and creep tests were again performed this time up to the required quarter of life rather than up to fracture. A creep test was also carried out up to $10 \%$ strain at $120 \mathrm{MPa}$ to observe the change in microstructure after necking just started.

\section{Microstructure Observation}

The microstructure of the as-received material and of the crept specimens at every stage were characterized by scanning electron microscopy (SEM) using both secondary electrons (SE) and back scattered electrons (BSE). Specimens were cut from the gauge section including the fractured area using a wire electrical discharge machine (EDM; Accutex Model 300i). Small areas from the uniform gauge section were cut from the crept samples at different life percentages as mentioned above. All the samples were polished to mirror finish using different grades of SiC papers starting from 600 grit to 1,200 grit and then continuing with Buehler micron pastes of 3 micron and 1 micron diamond particles. The final polishing was carried out using Buehler colloidal silica to obtain perfect mirror finish without any scratches. These samples were then etched using Villela's reagent to show the individual grains, grain boundaries, and the particles along the boundaries. Various etchants used in general for alloy steels were tried to reveal all the details. The best one was found to be Villela etchant, which helped reveal all the grain boundaries and precipitates very well. These etched samples were then inspected with an FEI XL-30 FEG ESEM (environmental scanning electron microscope) in SE mode with an accelerating voltage of $15 \mathrm{kV}$. Images were captured at magnifications of 3,500x and 8,000x to record the grains, cavities and precipitates from different areas of the samples. The images were captured from the fractured samples, at a distance of 100 microns from the fracture line up to 600 microns at different locations.

Electron backscatter diffraction is a SEM-based microstructural-crystallographic technique to measure the crystallographic orientations, which is applicable to any crystalline material. When the beam is scanned in a grid across a polycrystalline sample and the crystal orientation measured at each point, the resulting map will reveal the constituent grain morphology, orientations, boundaries and the texture present in the material. This map is called the orientation map. Electron Backscatter Diffraction (EBSD) analysis makes use of Kikuchi patterns to determine the crystallographic information. When an electron beam is incident on a crystalline material, electrons diffuse in all directions, forming a point source. Some of these electrons satisfy Bragg's diffraction condition for different sets of planes. These electrons diffract in each diffracting plane and form a cone corresponding to each set of planes (Kossel cones). Upon intersecting with a phosphor screen, these cones give the appearance of a band which is characteristic of the diffracting planes. For EBSD analysis, the sample is tilted $70^{\circ}$ from the horizontal as it enhances the fractions of electrons scattered from the sample. Since the width of these Kikuchi patterns is related to the inter-planar spacing and the angle between the bands relate to angles between the diffracting planes, these Kikuchi patterns can then be indexed to identify the planes and the zone axis. The Kikuchi patterns can be analyzed to get information on texture, grains, and grain boundaries and phase discrimination. Samples were polished for 30 
minutes on lapping cloth with colloidal silica to get better surface finish and minimize the disturbed layer since EBSD is quite sensitive and picks up even sub-surface deformation due to deeper scratches from prior polishing steps. EBSD mapping and misorientation analysis of grain boundaries of the as-received material as well as the crept materials at all stages was carried out using the FEI XL-30 FEG ESEM microscope with a TSL 4000 system.

The captured SEM images were analyzed using ImageJ for the cavities and precipitate size. ImageJ is a public domain, Java-based image processing program developed at the National Institutes of Health. ImageJ was designed with an open architecture that provides extensibility via Java plug-in and recordable macros. In each case, five images were analyzed. The images at a distance of 100 microns from the fracture surface were captured at five different locations and they were analyzed for cavities using ImageJ. The cavity density was then calculated by averaging the number of cavities over the total area in the images. These cavity density values are reported below and compared in the results section. Similarly, the size of carbides along the grain boundaries were also calculated for every stage of the crept samples using ImageJ. Grain size was determined using the ASTM standard for grain size measurement.

Transmission electron microscopy (TEM) analysis of the samples was carried out on an analytical Philips CM20 TEM with Gatan CCD, EDAX and nanoprobe mode operating at 200 $\mathrm{kV}$. TEM samples were prepared after cutting thin slices using a diamond saw followed by mechanical grinding to 100 microns by two techniques as described below. In the first method, slices sectioned from the samples were thinned down to $100 \mu \mathrm{m}$ by mechanical polishing and then $3 \mathrm{~mm}$ diameter discs were punched out. A dimpling wheel with diamond paste media ranging from $6 \mu \mathrm{m}$ to $1 \mu \mathrm{m}$ was then used to thin the center of this disc to a thickness of $\approx 20 \mu \mathrm{m}$. The sample was then polished using a polishing wheel using non-crystallizing colloidal silica $(0.02 \mu \mathrm{m})$ to a mirror finish. Samples were polished until all scratches were removed. This was done to remove any deformation layer introduced by sample preparation. After this, the $3 \mathrm{~mm}$ disc was ion-milled until a perforation was detected using a laser beam device. A Fischione Instruments Model 1010 ion mill was used. During ion milling, the center of the sample is thinned by sputtering using inert gas (Ar) ions. As soon as the perforation is achieved, the milling is terminated by detection of laser light. Two ion sources are used to thin samples faster, from opposing surfaces of the disc. The sample was also cooled using liquid nitrogen to avoid any artifacts and continuously oscillated during ion milling, to have a larger thin area. The following ion milling parameters were used: current: $5 \mathrm{~mA}$, voltage $5 \mathrm{~V}$, sample rotation $60^{\circ}$, beam incident angle $14^{\circ}$, stage temperature $-80^{\circ} \mathrm{C}$

In the second method, slices were sectioned from the area of interest and thinned down by mechanical grinding to a thickness of $\approx 100 \mu \mathrm{m}$. Discs of $3 \mathrm{~mm}$ diameter were then punched using a disc punch. The discs were polished on SiC paper to remove burr, which can form from the punching and then further thinned down to $\approx 80 \mu \mathrm{m}$. The sample was then electrolytically polished using a twin jet electro polisher (Fischione Model 110 Twin-Jet Electro polisher). In this electro polisher two jets of electrolyte are incident on the opposite sides of disc, near the center and appropriate voltage is applied to the sample through a platinum anode to polish the sample until a hole is produced near the center of sample. The hole is detected by a photocell which stops the polishing as soon as the hole is made. The areas around that hole are electron transparent and used for analysis. The advantage of polishing with this method is that no mechanical force is used on the thin sample and hence in a case where plastic deformation is to be studied one can eliminate sample preparation as a source of deformation. The following conditions were used for polishing the samples in this study: electrolyte $10 \%$ Perchloric acid + $90 \%$ ethanol, voltage $-30 \mathrm{~V}$, temperature of the electrolyte $-40{ }^{\circ} \mathrm{C}$. 


\section{Hardness Measurement}

Prior to microhardness measurements, samples were polished to mirror finish using Buehler colloidal silica solution. Microhardness measurements were made using a Knoop indenter on a LECO microhardness tester at $500 \mathrm{~g}$ load for 15 seconds on the polished surface. Knoop microhardness tests were conducted on all the samples to observe the hardness variation trend as the creep proceeds at various stress levels and also as the hardness varies from the fractured area to the non-necked gauge area. The trends are plotted and compared in the results section.

\subsection{Inspection results and discussion}

In this section we will present the results of our metallurgical investigation and discuss the results.

\section{Initial Microstructure}

A SEM micrograph of the microstructure of the as-received sample which was rolled and heat treated is shown in the Figure 41. The initial microstructure is tempered martensite, composed of laths of martensite with a decoration of carbides along its boundaries as well as inside the grains and sub-grains. The grain size is around $5 \mu \mathrm{m}$.

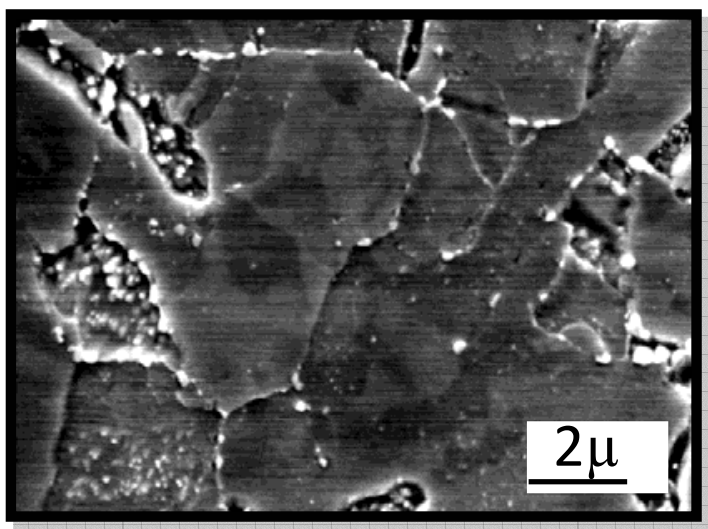

Fig. 41. SEM image of as received modified 9Cr-1Mo steel.

The EBSD orientation map of the as-received steel is shown in the Figure 42. The EBSD map shows a lath structure of the martensite and also a uniform sub-grain structure. The percentage of low-angle and high-angle grain boundaries in the as received structure are $43 \%$ and $57 \%$, respectively. On an average each grain consists of three sub-grains.
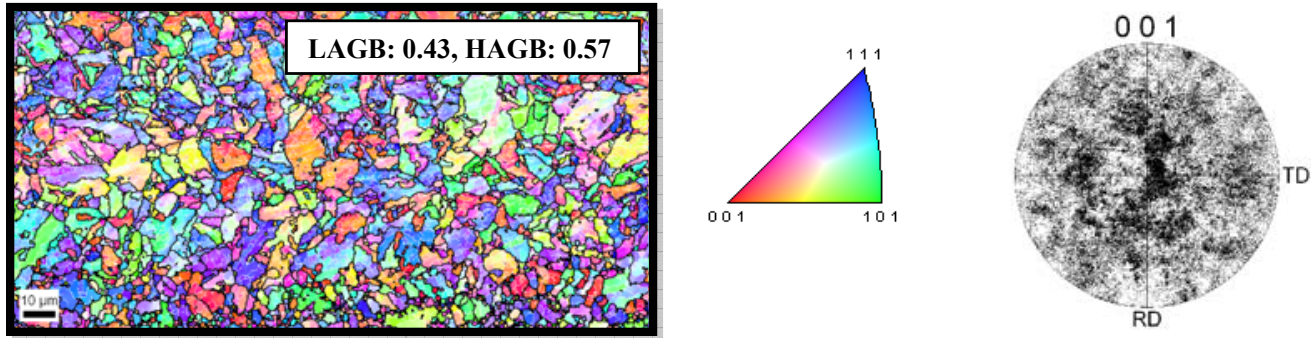

Fig. 42. EBSD orientation map and pole figure of the as received modified 9Cr-1Mo steel. 
Figure 43 shows bright field (BF) TEM images of the as-received steel. The ferrite lath and carbide particles on the grain boundary triple points can be seen in the TEM images. As suggested by the images in Figure 43, the as-received material has microstructure characterized by micro grains and uniform sub-grains with carbides on the triple points and grain boundaries. Free dislocations can be seen inside some of the sub-grains.
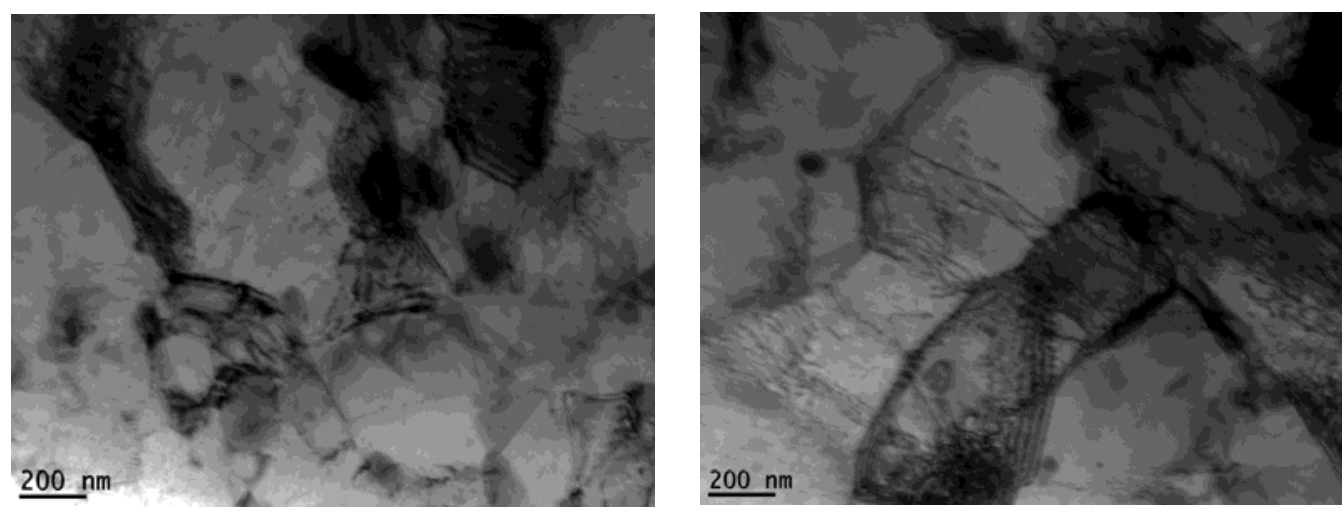

Fig. 43. TEM images of as received modified 9Cr-1Mo steel.

\section{Creep Test Results}

Creep tests were performed at various stress levels ranging from $80 \mathrm{MPa}$ to $140 \mathrm{MPa}$ and at different life intervals ranging from $25 \%$ of life to $75 \%$ of creep life and until fracture. All creep tests were performed at a fixed temperature of $650{ }^{\circ} \mathrm{C}$. Figure 44 shows the combined creep curves (creep strain versus time) at all the stress levels. The rupture strain of all the samples was calculated to be $\approx 50 \%$ manually from the dimensions of the fractured samples. Table 3 indicates the minimum creep rates and creep life at different stress levels according to the creep curves. It can be seen that the rupture life increases exponentially with a decrease of $20 \mathrm{MPa}$ of stress at every step. It can also be noticed that the minimum creep rate is much lower at the lower stress.

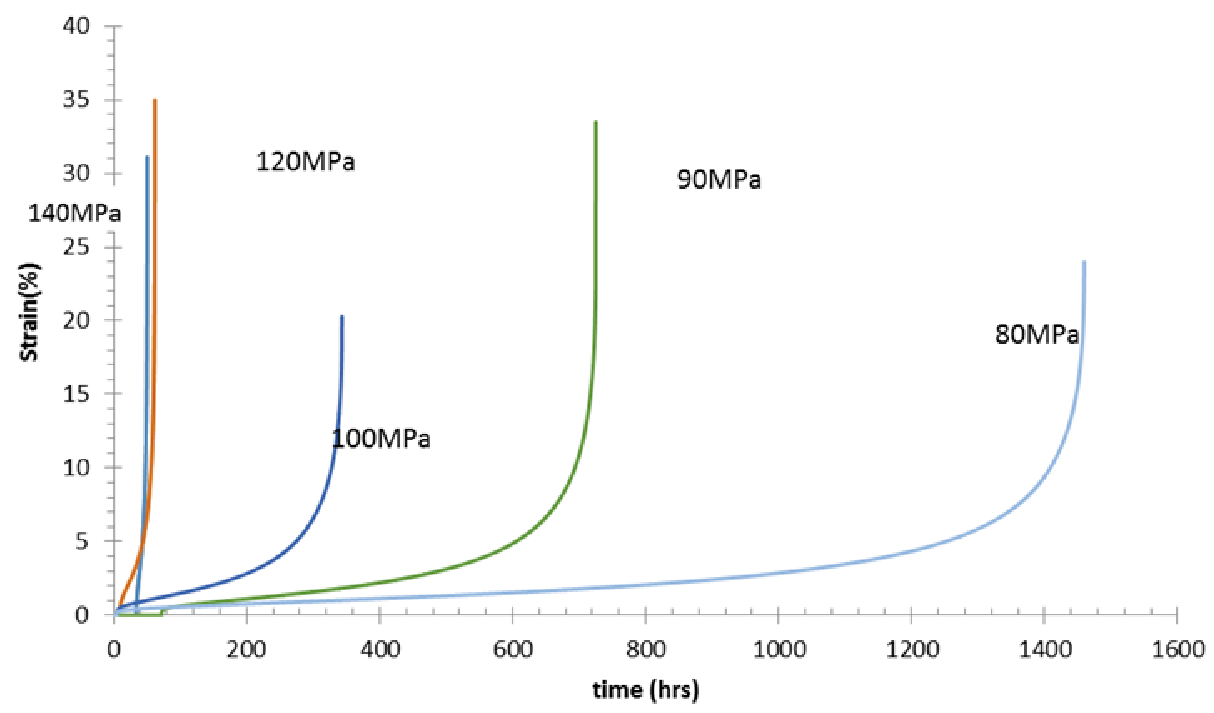

Fig. 44. Creep curves of modified $9 \mathrm{Cr}-1 \mathrm{Mo}$ steel at $650^{\circ} \mathrm{C}$ and different stress levels ranging from 80-140 MPa. 
Table 3 Creep data at all the stress levels.

\begin{tabular}{|c|c|c|}
\hline $\begin{array}{c}\text { Stress } \\
{[\mathrm{MPa}]}\end{array}$ & $\begin{array}{c}\text { Rupture Time } \\
{[\mathrm{hrs}]}\end{array}$ & $\begin{array}{c}\text { Minimum Creep Rate } \\
{[\% / \mathrm{hr}]}\end{array}$ \\
\hline 140 & 50.2 & 0.5 \\
\hline 120 & 58 & 0.07 \\
\hline 100 & 343 & 0.016 \\
\hline 90 & 725.5 & 0.009 \\
\hline 80 & 1459.7 & 0.0078 \\
\hline
\end{tabular}

Figure 45 shows a log- $\log$ plot of the minimum creep rate versus stress. The linear trend line of the data is plotted and its matching parameters are also indicated. Dispersion strengthened alloys are characterized by exponents higher than 7 and high activation energies. The slope, which is the stress exponent, $n \approx 9$ indicates that the mechanism of creep is dislocation creep. As mentioned earlier, creep tests were also conducted at different percentages $(25 \%, 50 \%$, and $75 \%)$ of the total life at $120 \mathrm{MPa}$ and $80 \mathrm{MPa}$. The creep curves of these cases are plotted in Figure 46 and the creep data is analyzed. In Figure 46, the creep behavior, i.e., strain versus time graphs at $120 \mathrm{MPa}$ at four life fractions as the creep proceeds, can be seen. From the creep behavior we can observe that up to $75 \%$ of the lifetime, the creep strain is as low as $4 \%$ but in the last quarter of the life there is a drastic increase in strain until fracture. Hence, maximum deformation from necking to failure takes place in the final quarter of life, representing a catastrophic failure.

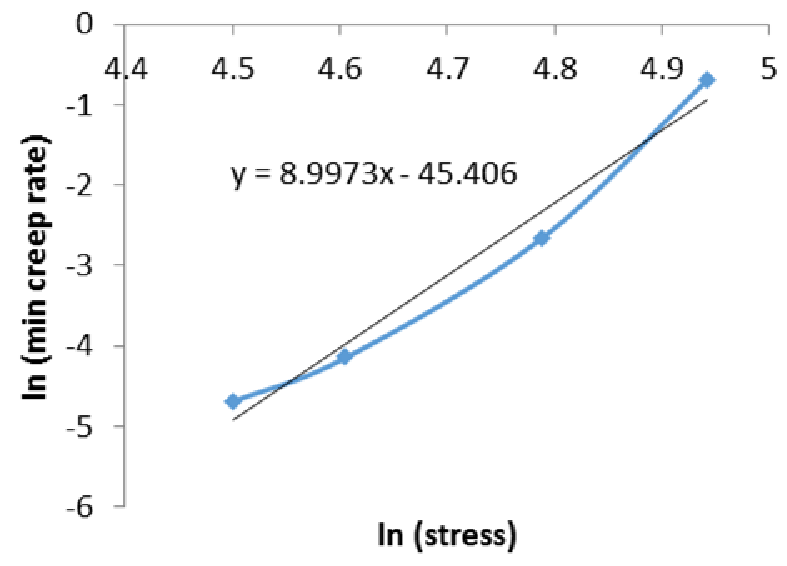

Fig. 45. Log-log plot of minimum creep rate vs. stress. 


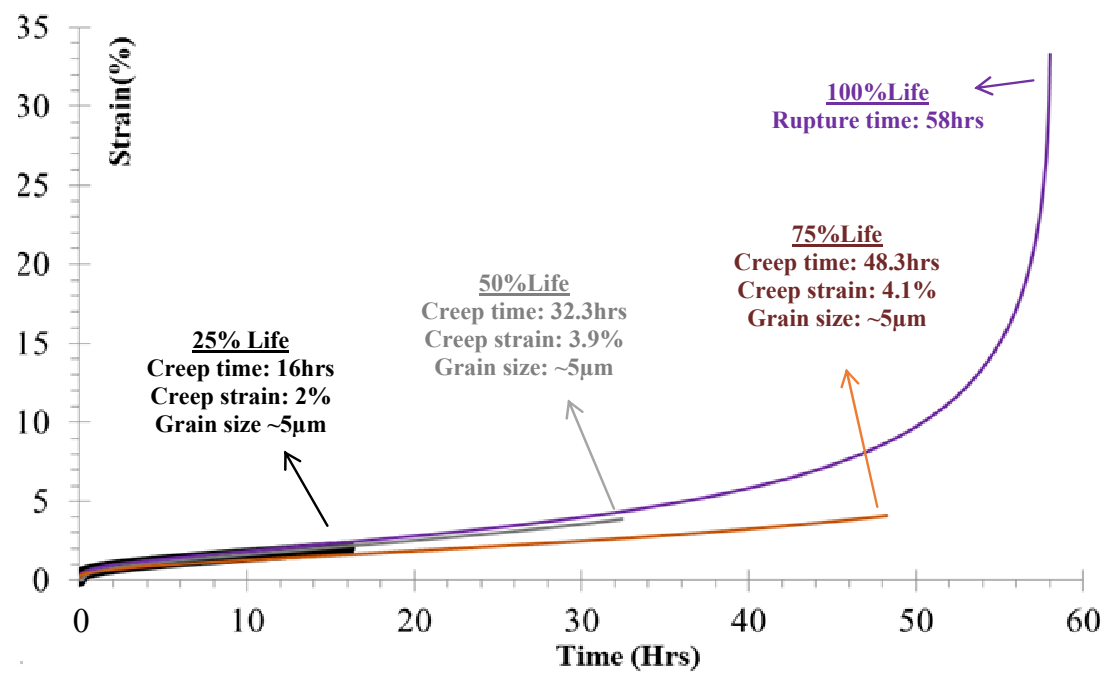

Fig. 46. Creep behavior at $120 \mathrm{MPa}, 650^{\circ} \mathrm{C}$.

Figure 47 shows the strain versus time graph as the creep proceeds at $80 \mathrm{MPa}, 650{ }^{\circ} \mathrm{C}$ at four life fractions similar to above. It can be observed from the creep behavior in this case as well that up to $75 \%$ of the lifetime, the creep strain is as low as $3 \%$ but in the last quarter of the life there is a drastic increase in strain until fracture. Hence, maximum deformation from necking to failure takes place in the final quarter of life.

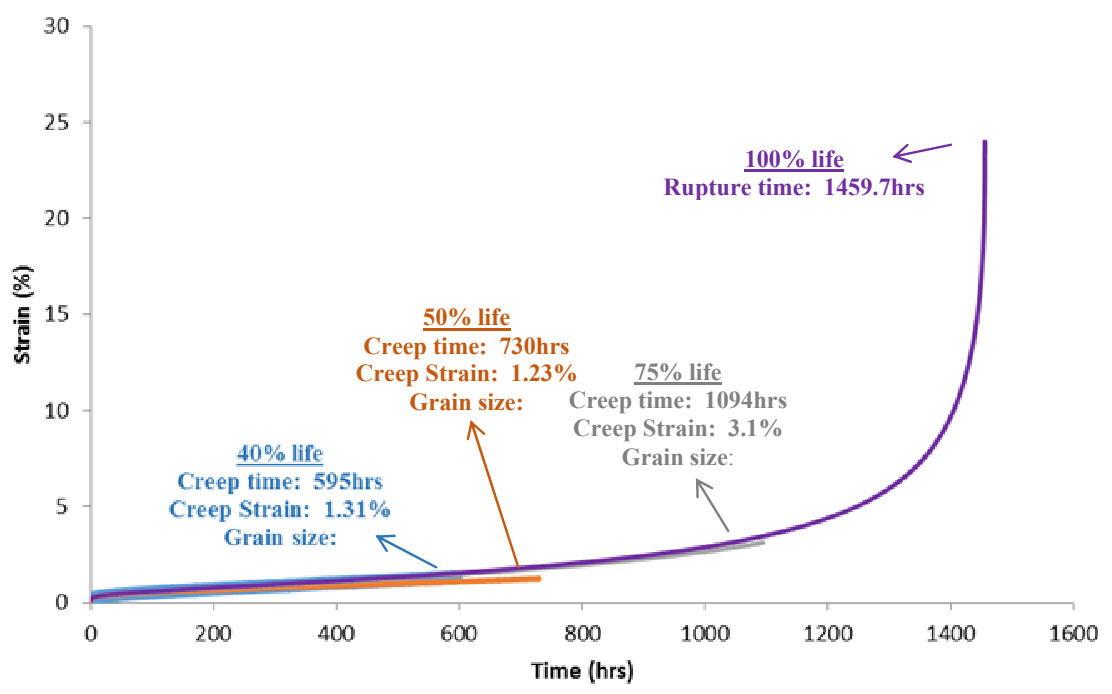

Fig. 47. Creep behavior at $80 \mathrm{MPa}, 650^{\circ} \mathrm{C}$.

Grains, sub-grains, grain boundaries, cavities and particles

Scanning electron microscopy (SEM) and electron backscattered diffraction (EBSD) was used to characterize creep in modified $9 \mathrm{Cr}-1 \mathrm{Mo}$ steels at various stress and time levels. Various SE (secondary electrons) mode SEM images were taken and analyzed for cavities and carbide particles during and after creep. Figure 48 shows the cavities near the fracture area. All the images were captured at a magnification of 3,500x from a distance of $100 \mu \mathrm{m}$ from the fracture 
line. It can be inferred that the number density of cavities at the vicinity of the fracture area is about three times higher when crept at $140 \mathrm{MPa}$ as compared to $120 \mathrm{MPa}$. Table 4 gives the cavity densities calculated using the ImageJ software and verified manually for all stress levels. Cavity density depends on the carbide density as the carbides at grain boundaries are the preferential sites for cavity nucleation. We can see higher carbide density and hence higher cavity density at $140 \mathrm{MPa}$.

Table 4 Creep data at five stress levels.

\begin{tabular}{|c|c|}
\hline $\begin{array}{c}\text { Stress } \\
{[\mathrm{MPa}]}\end{array}$ & $\begin{array}{c}\text { Cavity Density } \\
{\left[\text { number } / \mathrm{mm}^{2}\right]}\end{array}$ \\
\hline 140 & 33,992 \\
\hline 120 & 12,286 \\
\hline 100 & 53,149 \\
\hline 90 & 42,553 \\
\hline 80 & 51,023 \\
\hline
\end{tabular}

The creep time is much longer at $80 \mathrm{MPa}, 90 \mathrm{MPa}$, and $100 \mathrm{MPa}$ when compared to higher stress levels. Near-fracture areas at these stress levels have a very similar carbide density. This can be explained with the role of exposure time and carbide density. Though the carbide density is lower at $80 \mathrm{MPa}$, the exposure time is exponentially higher when compared to that at 140 $\mathrm{MPa}$, both at $650^{\circ} \mathrm{C}$. The higher the exposure time, the higher the time for the nucleation and movement of voids and formation of cavities will be. Hence, the samples fractured at $80 \mathrm{MPa}$ to $100 \mathrm{MPa}$ have a similar cavity density owing to exposure time and carbide density, respectively.

The carbides decorating the grain boundaries of the ferrite lath after creep from an area that is $100 \mu \mathrm{m}$ from the fracture surface at all the stress levels are compared with the as-received structure of the modified $9 \mathrm{Cr} 1 \mathrm{Mo}$ steel in Figure 49. The carbide sizes in each case are measured using the ImageJ software averaged over five images taken from the same distance of $100 \mu \mathrm{m}$ from the fracture area. The average carbide size in each case is listed in Table 5 . 


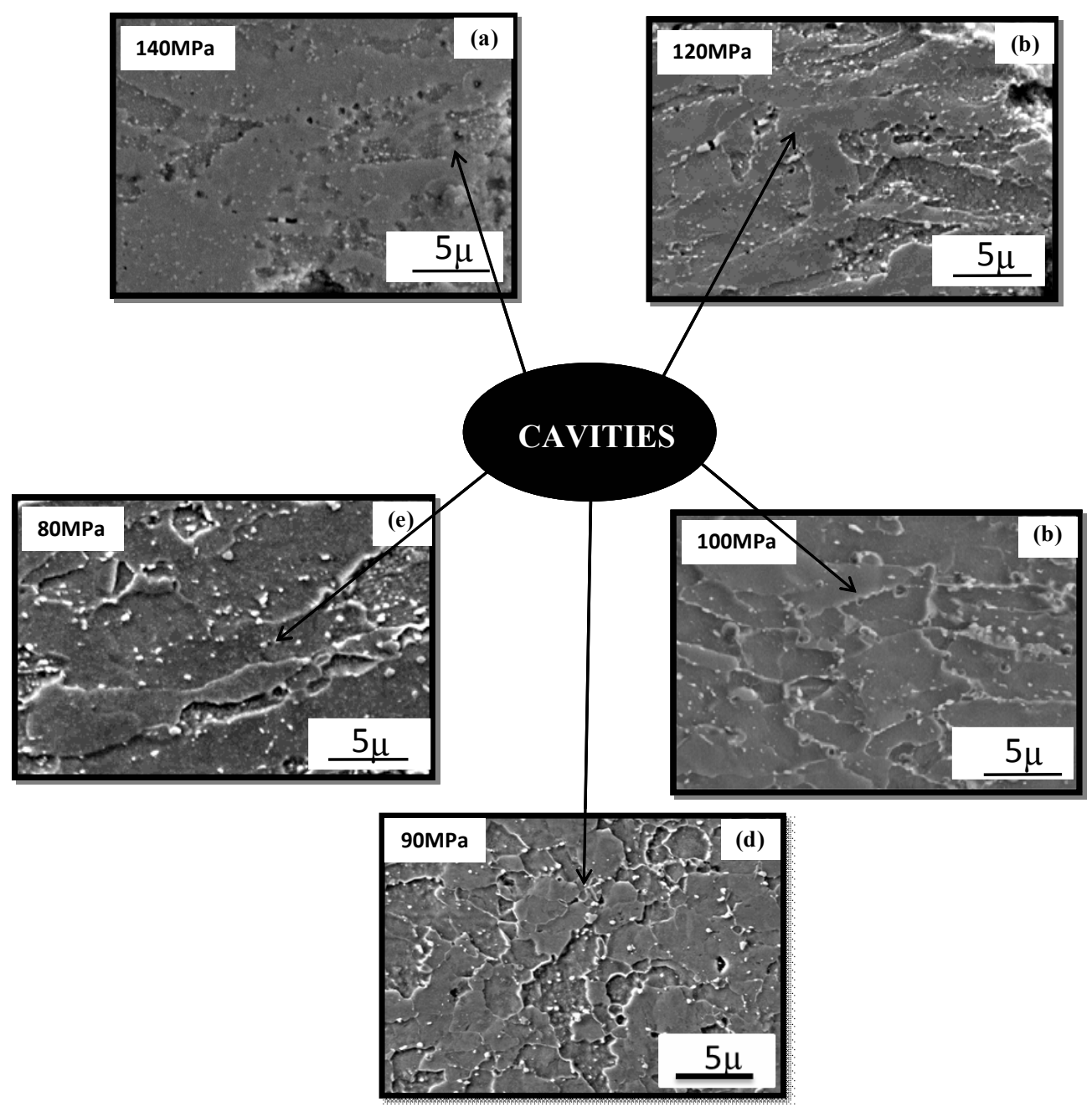

Fig. 48. Cavities from the fracture area at various stress levels.

To evaluate the growth of carbides as the creep proceeds, SEM images at each quarter of creep life were also captured. Figure 50 shows the growth of carbides as the creep proceeds at $120 \mathrm{MPa}$. No necking was observed until $75 \%$ of creep life occurred and hence no cavities were observed until the final quarter of life. Similarly, Figure 51 shows the carbides as the creep proceeds at $80 \mathrm{MPa}$ at (a) $25 \%$ life, (b) $50 \%$ life, (c) $75 \%$ life, and (d) fracture. 
Table 5 Average carbide size at all stress levels.

\begin{tabular}{|c|c|}
\hline $\begin{array}{c}\text { Stress } \\
{[\mathrm{MPa}]}\end{array}$ & $\begin{array}{c}\text { Average Carbide Size } \\
{[\mathrm{nm}]}\end{array}$ \\
\hline as received & 200 \\
\hline 140 & 193 \\
\hline 120 & 223 \\
\hline 100 & 258 \\
\hline 90 & 298 \\
\hline 80 & 324 \\
\hline
\end{tabular}

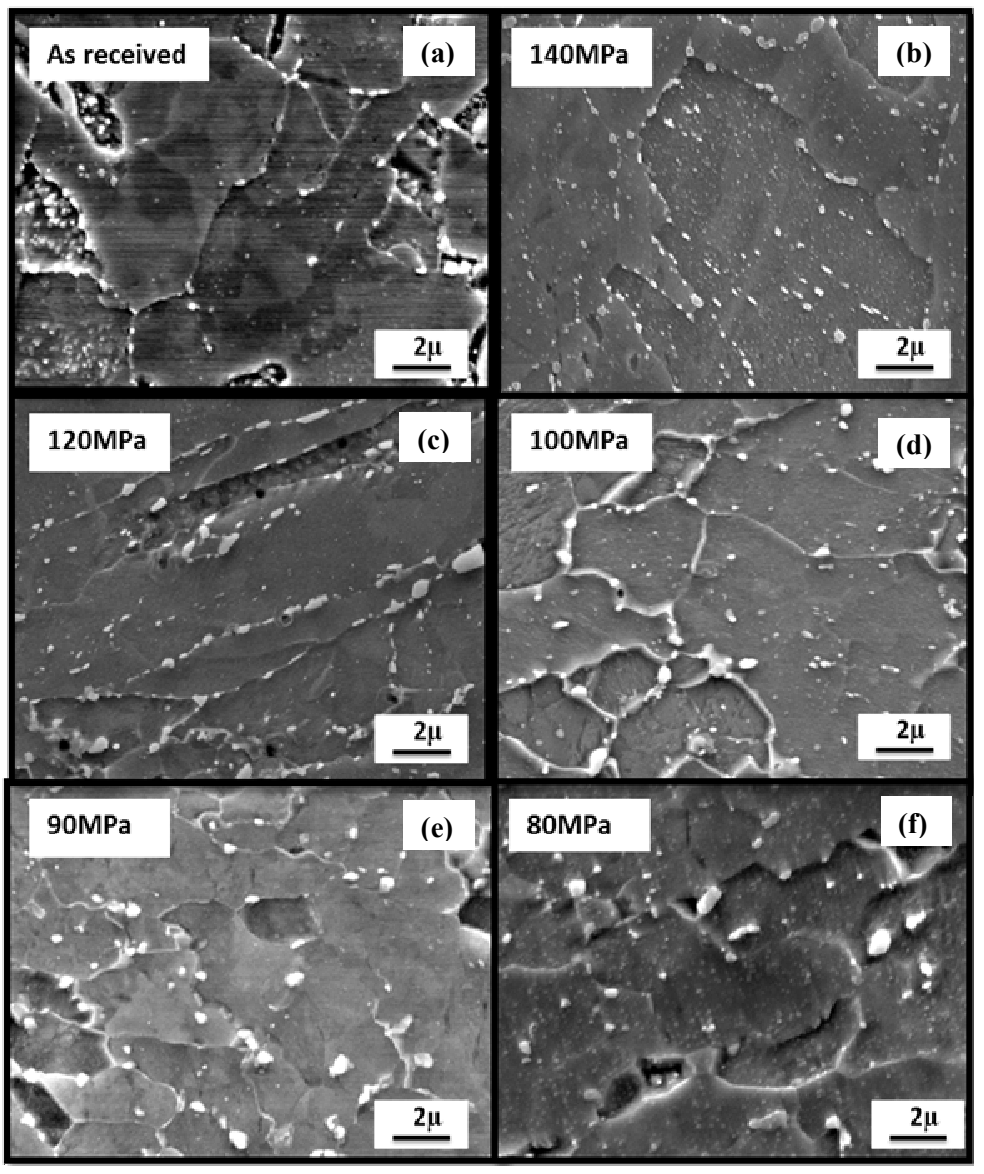

Fig. 49. Carbides along the grain boundaries of as received and at all stress levels. 

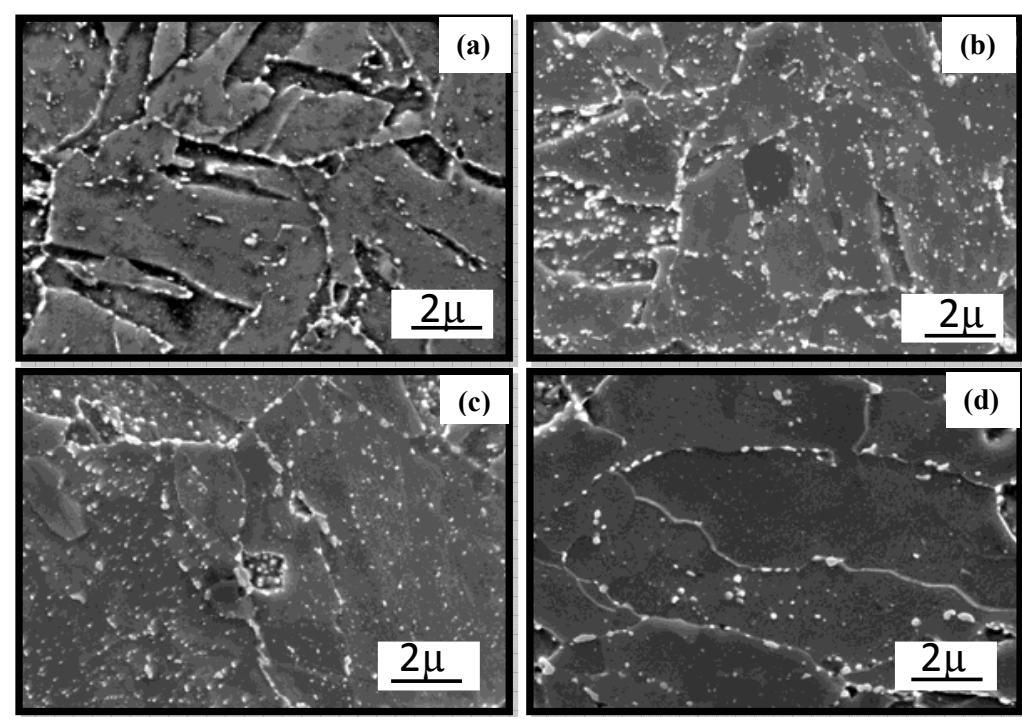

Fig. 50. Carbides as the creep proceeds at $120 \mathrm{MPa}$ at (a) $25 \%$ life, (b) $50 \%$ life, (c) $75 \%$ life, and (d) fracture.
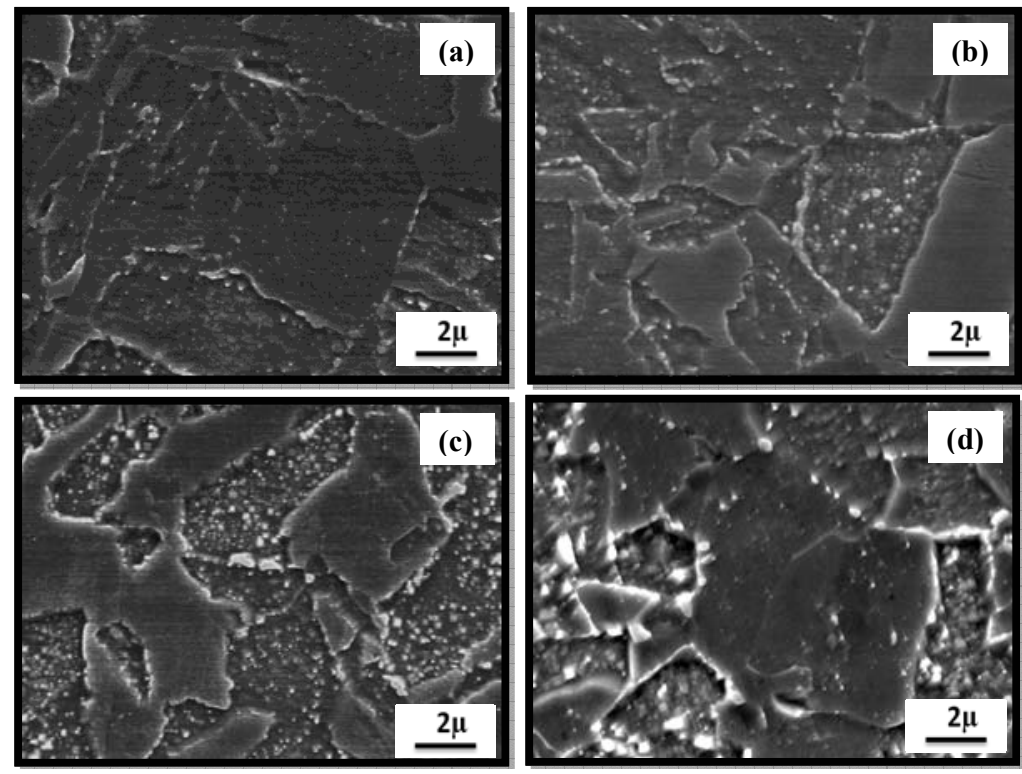

Fig. 51. Carbides as the creep proceeds at $80 \mathrm{MPa}$ at (a) $25 \%$ life, (b) $50 \%$ life, (c) $75 \%$ life, and $(d)$ fracture.

Growth of carbides was observed but the growth was very slow due to the very high stress level and less time for diffusion of atoms to help grow the carbides. The measurement of Vickers hardness values were carried out at the gauge area of the crept specimen at all stages during the creep of the steel at $120 \mathrm{MPa}$ and $650{ }^{\circ} \mathrm{C}$. The hardness was then plotted as a function time along with the carbide size. The decrease in hardness and the increase in carbide size as the creep proceeds at $120 \mathrm{MPa}$ can be seen clearly from the graph in Figure 52. 


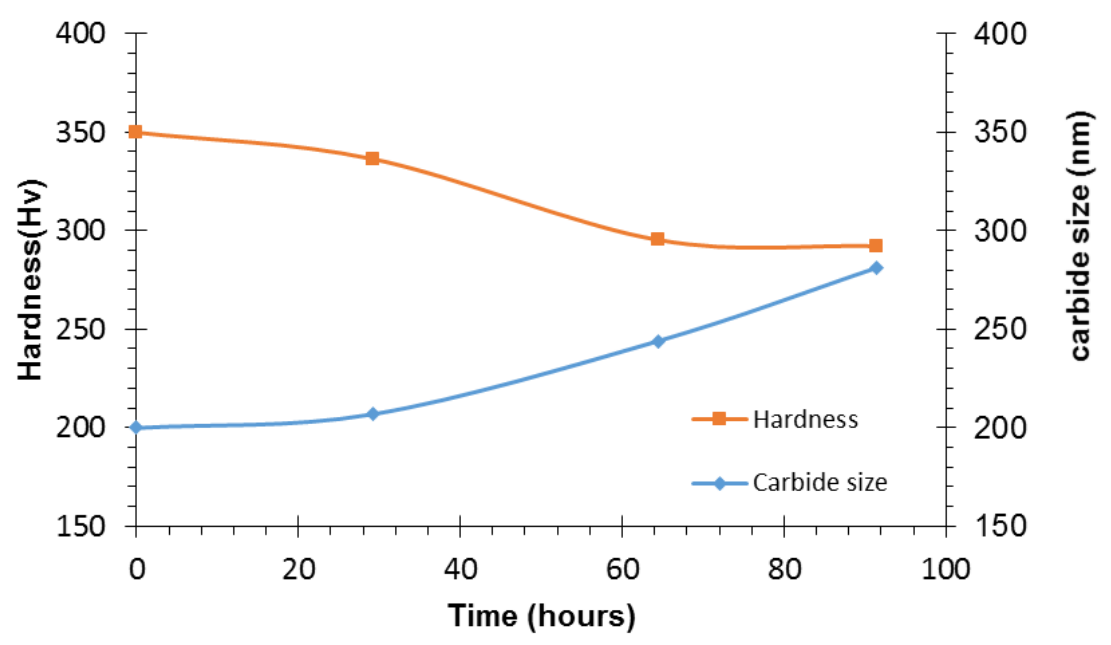

Fig. 52. Change of hardness and carbide size as creep proceeds at $120 \mathrm{MPa}, 650{ }^{\circ} \mathrm{C}$.

Electron backscatter diffraction was also used to characterize the grains, sub-grains, grain boundaries and evaluate grain sizes. EBSD orientation scans were obtained at every stress level and every stage of the crept samples. Figure 53 shows the typical EBSD orientation maps from all stages of creep at $120 \mathrm{MPa}$ starting from $25 \%$ life to fracture. The pole figure and orientation imaging micrographs (OIMs) indicate random orientation of grains and no specific alignment of grains. The fractions of low angle grain boundaries (LAGB) and high angle grain boundaries (HAGB) are indicated. It can be seen that the fraction of LAGB increases as the creep proceeds, which indicates decrease in sub-grain size and increase in grain size as the creep proceeds. Figure 54 shows the EBSD orientation maps as the creep proceeds at $80 \mathrm{MPa}$, starting from $25 \%$ life to fracture The same trend as observed above can be seen here as well. The fraction of low angle grain boundaries increases as creep proceeds. Elongated grains can be seen at fracture but not before $75 \%$ of life which proves the fact that damage accumlation and accelerated creep occurs in the final quarter of life. Formation and growth of sub-grain boundaries is the main microstructure evolution mechanism at lower stress levels.

Orientation maps as a function of strain, which is again a function of distance from the fracture area varying from $100 \mu \mathrm{m}$ to $6,000 \mu \mathrm{m}$, can be seen in Figures 55, 56, 57 and 58. As the strain varies from $\approx 8 \%$ to $\approx 50 \%$, we can see that the fraction of LAGB also increases in all the cases, which indicates formation of more subgrains that can also be evidently seen from the OIMs. At lower stress microstructure and sub-structure is observed by EBSD technique to verify the grain size, morphology and grain boundary characters. The sub-grain size is about the same and around 2.5 microns near the fractured areas. It is obvious that by decreasing the level of stress the fraction of the newly formed recrystallized grains near the fracture surface is decreased notably. The grains are elongated and forming sub-structure. The degree of elongation is lower than at higher stress levels. This is mostly attributed to decrease in strain rate at lower stresses. Formation and growth of sub-grain boundaries is the main microstructure evolution mechanism at lower stress levels. The creep mechanism is still dislocation creep at these levels of stress.

At middle stress level the microstructure and sub-structure are also observed by EBSD technique to verify the grain size, morphology and grain boundary characters. The grains are not elongated before $75 \%$ of creep life. Formation and growth of sub-grain boundaries is the main 
microstructure evolution mechanism at lower stress levels. The creep mechanism is still dislocation creep at these levels of stress. The average grain size is around 5 microns.
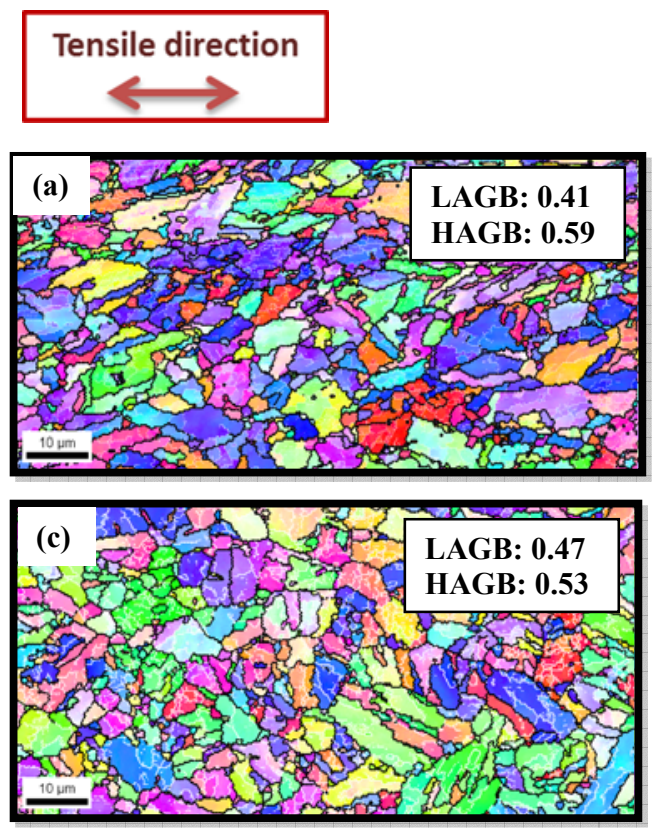
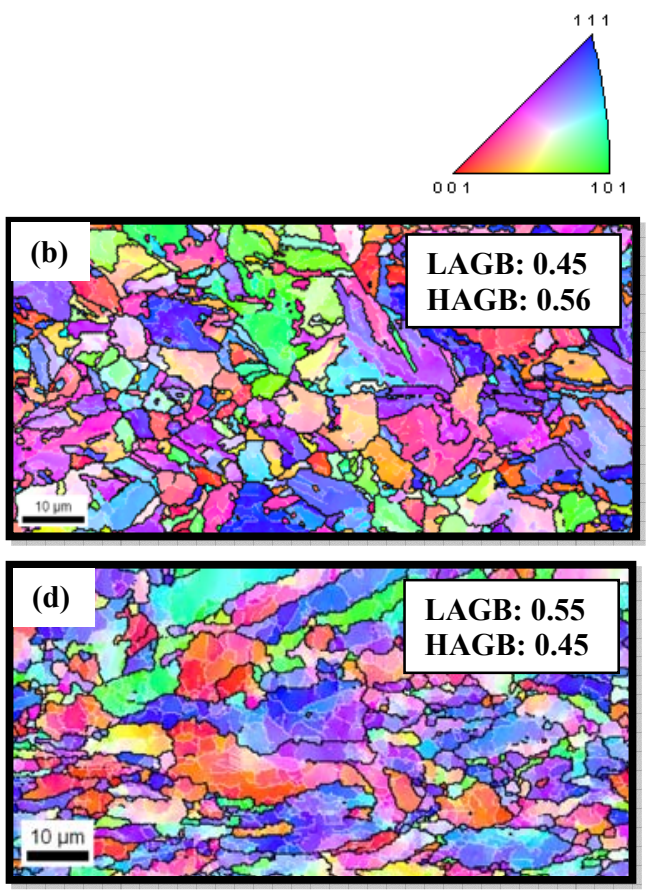

Fig. 53. EBSD OIM as the creep proceeds at $120 \mathrm{MPa}$ and (a)25\% life, (b) $50 \%$ life, (c) $75 \%$ life, and (d) fracture.
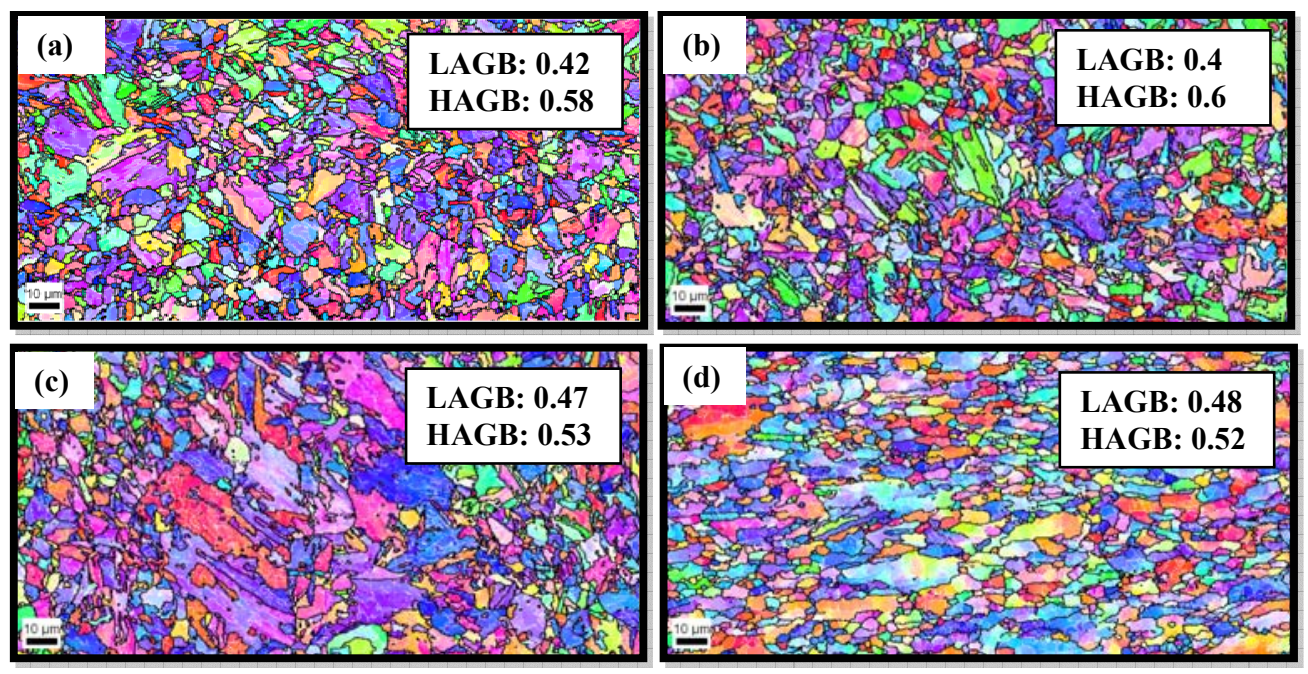

Fig. 54. EBSD OIM as the creep proceeds at $80 \mathrm{MPa}$ and (a)25\% life, (b) 50\% life, (c) $75 \%$ life, and (d) fracture. 


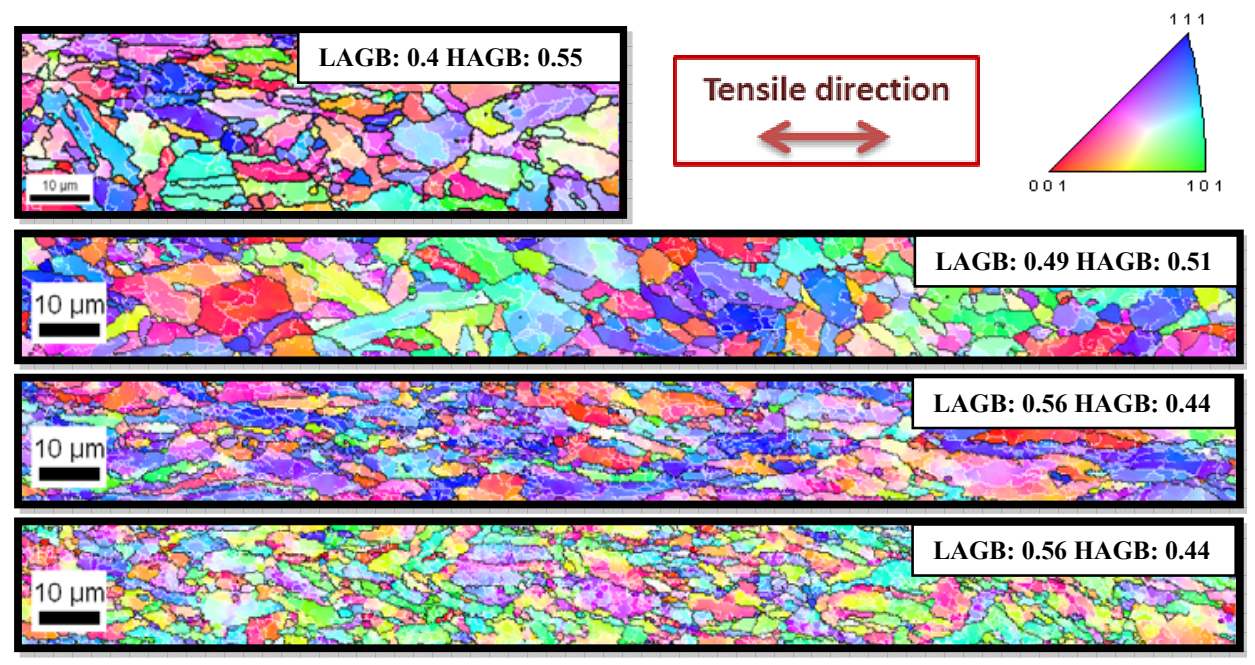

Fig. 55. EBSD OIM as a function of strain at $140 \mathrm{MPa}$ from (a) $\approx 8 \%$ to (d) $\approx 50 \%$.
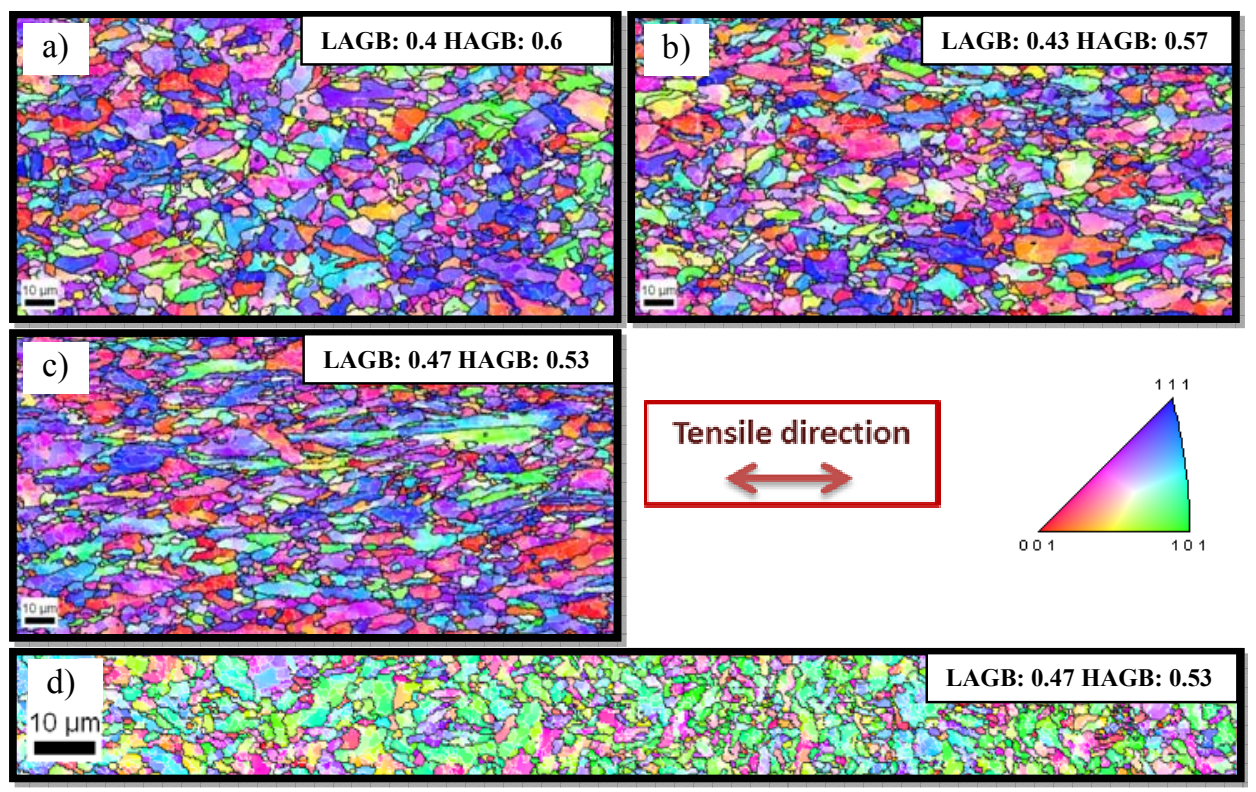

Fig. 56. EBSD OIM as a function of strain at $120 \mathrm{MPa}$ from (a) $\approx 8 \%$ to (d) $\approx 50 \%$. 

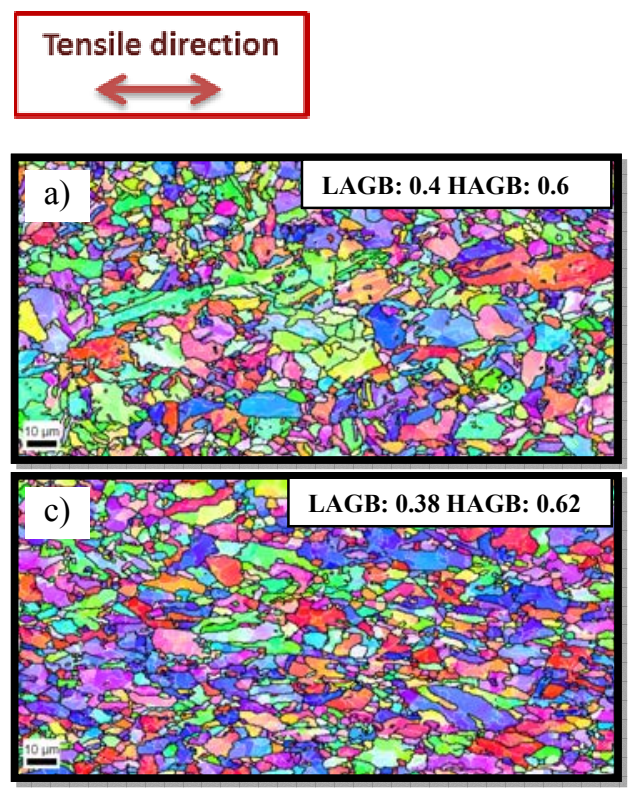
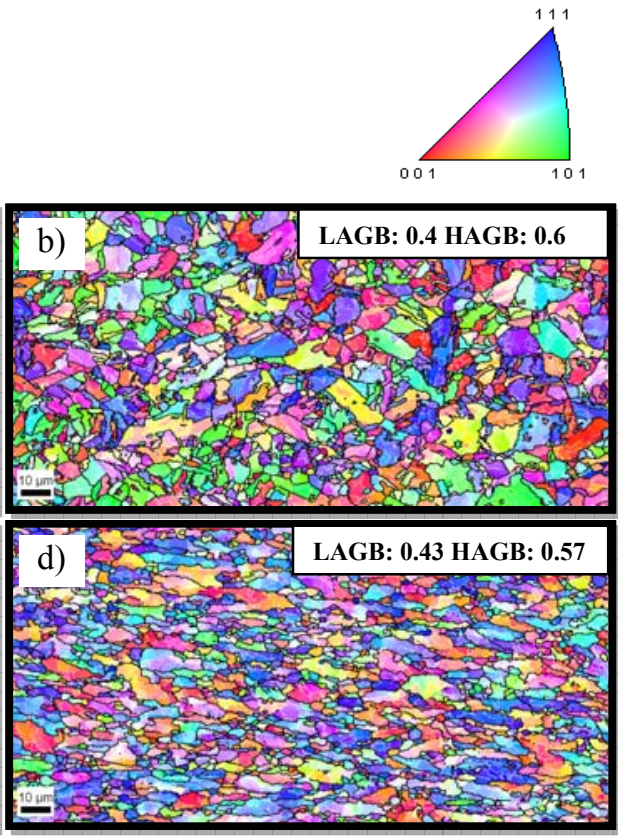

Fig. 57. EBSD OIM as a function of strain at $100 \mathrm{MPa}$ from (a) $\approx 8 \%$ to $(\mathrm{d}) \approx 50 \%$.
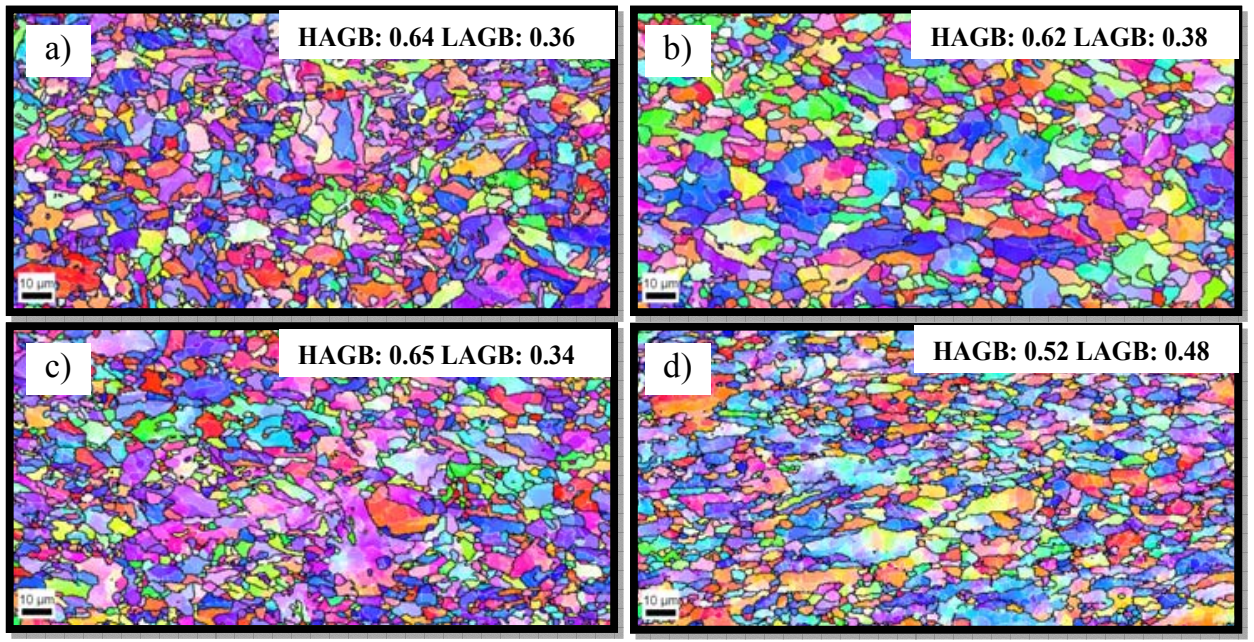

Fig. 58. EBSD OIM as a function of strain at $80 \mathrm{MPa}$ from (a) $\approx 8 \%$ to $(\mathrm{d}) \approx 50 \%$.

Table 6 shows the fraction of low angle grain boundaries near the fracture site at $\approx 50 \%$ strain level at different stress levels. It can be seen that the LAGB values are almost the same at lower stress levels but they shoot up at $140 \mathrm{MPa}$ where more sub-grain boundaries form, but there is not enough time to increase the angle of misorientation to form HAGBs. On the other hand, at lower stress levels there is enough time for the rotation of sub-grains and increasing angle of misorientation to form HAGBs. Table 7 shows the values of low-angle and high-angle grain boundaries at $\approx 8 \%$ strain which can be obtained at a distance of almost $6,000 \mu \mathrm{m}$ from the fracture site at all the stress levels. This is the area where the gage section is uniform below the 
necked area after the fracture. It can be seen that despite being under different levels of stress and different amount of time at $650{ }^{\circ} \mathrm{C}$ at each particular stress level, the LAGB value remains almost the same. The fraction of low-angle grain boundaries formed is always around $40 \%$ at the strain level of $\approx 8 \%$.

Table 6 LAGB, HAGB and grain size values at different stress levels near the fracture site at $\approx 50 \%$ strain.

\begin{tabular}{|c|c|c|}
\hline $\begin{array}{c}\text { Stress } \\
{[\mathrm{MPa}]}\end{array}$ & $\begin{array}{c}\text { LAGB } \\
{[\%]}\end{array}$ & $\begin{array}{c}\text { HAGB } \\
{[\%]}\end{array}$ \\
\hline 80 & 48 & 52 \\
\hline 90 & 51 & 49 \\
\hline 100 & 43 & 57 \\
\hline 120 & 47 & 53 \\
\hline 140 & 56 & 44 \\
\hline
\end{tabular}

Table 7 LAGB, HAGB and grain size values at different stress levels $6,000 \mu \mathrm{m}$ from fracture site at $\approx 8 \%$ strain.

\begin{tabular}{|c|c|c|c|}
\hline $\begin{array}{c}\text { Stress } \\
{[\mathrm{MPa}]}\end{array}$ & $\begin{array}{c}\text { LAGB } \\
{[\%]}\end{array}$ & $\begin{array}{c}\text { HAGB } \\
{[\%]}\end{array}$ & $\begin{array}{c}\text { Grain Size } \\
{[\text { microns }]}\end{array}$ \\
\hline 80 & 36 & 64 & 4.75 \\
\hline 90 & 42 & 58 & 4.4 \\
\hline 100 & 40 & 60 & 4 \\
\hline 120 & 40 & 60 & 4.5 \\
\hline 140 & 40 & 60 & 5.55 \\
\hline
\end{tabular}

\section{Observations and comparison with transmission electron microscopy}

TEM observations were made at the highest and lowest stress levels of $140 \mathrm{MPa}$ and $80 \mathrm{MPa}$ from the gage and grip sections of each fractured specimen. The grip section is the part of the sample that undergoes only heat treatment at $650{ }^{\circ} \mathrm{C}$, which is the temperature at which creep is carried out whereas the gage section is where the stress is applied and the effect of both stress and temperature can be seen. Figure 59(a) shows the uniform sub-grain structure of the heat 
treated material with a huge carbide particle present at the grain boundary. Figure 59(b) shows the magnified carbide present exactly at the grain boundary triple point. When the images in Figure 59 are compared with those in Figure 43 from the as-received material, it can be confirmed that after heat treatment for almost 1,500 hours, the grains and sub-grains increased in size and the carbides along the grain boundaries and sub-grain boundaries also increased in size. Figures 59(c) and 59(d) also show the uniform sub-grains with dislocations. The effect of heat treatment is seen in the form of decrease in dislocation density as compared to the as received structure.
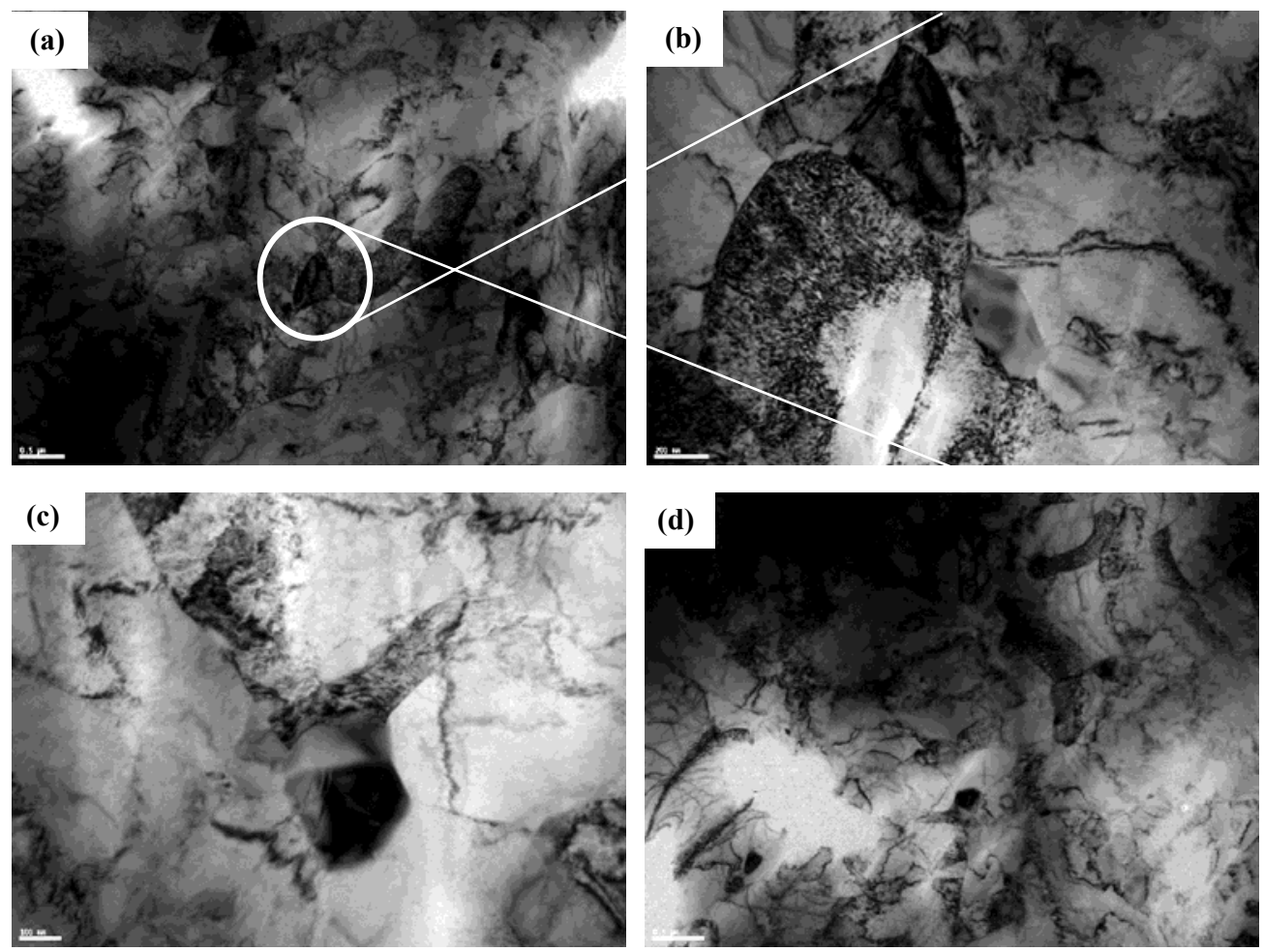

Fig. 59. TEM images of modified $9 \mathrm{Cr}-1 \mathrm{Mo}$ steel heat treated at $650{ }^{\circ} \mathrm{C}$ for about 1,462 hours.

Figure 60 shows the TEM images from the grip section of the $140 \mathrm{MPa}$ sample, which is equivalent to heat treatment at $650^{\circ} \mathrm{C}$ for about 75 hours. When compared with the above images, we can see that sub-grains are much smaller here and also the carbides are smaller than those heat treated for about 1,500 hours. This proves the fact the carbides and sub-grains both grow enormously under the effect of just time. It can be seen that dislocation density in this case is higher compared to those in Figure 59. 

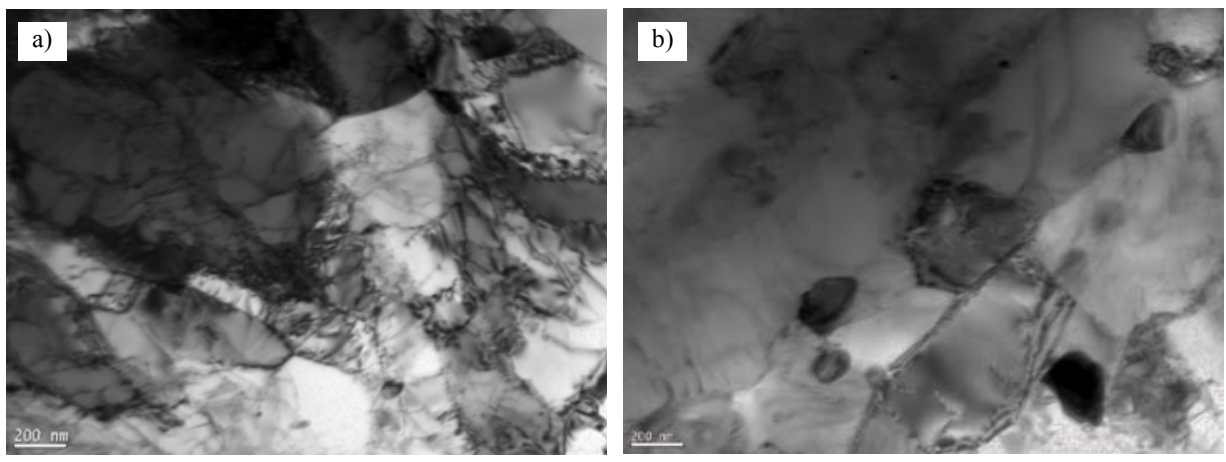

Fig. 60. TEM images of modified $9 \mathrm{Cr}-1 \mathrm{Mo}$ steel heat treated at $650{ }^{\circ} \mathrm{C}$ for about 75 hours.

TEM images from the uniformly elongated gauge section of the crept specimen at a stress level of $80 \mathrm{MPa}$ can be seen in Figure 61. When compared to the as-received material, dislocations are observed at this stage and the sub-grain size almost remains the same. Grain boundary pinning by carbide particles can be clearly seen Figure 61(b).
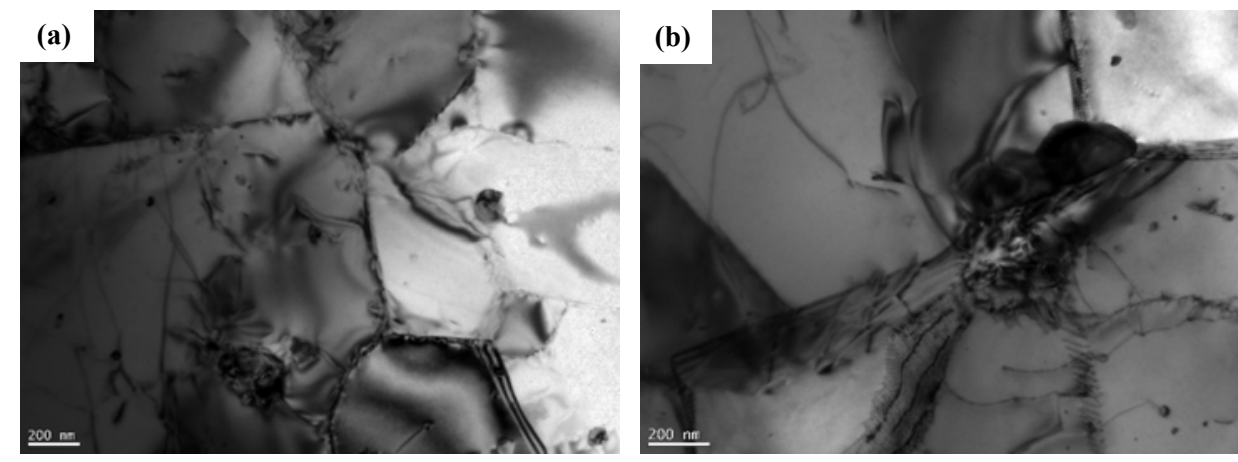

Fig. 61. TEM images from the gauge section after creep at $80 \mathrm{MPa}$.

Figure 62 shows images from the gauge area of a sample crept at $80 \mathrm{MPa}$. The images show the interaction of dislocations with the small carbides inside the lath. These carbides also help pin the sub grain boundaries. Pinning by small carbides in the matrix during the creep is one of the main reasons for the high creep strength in these materials.
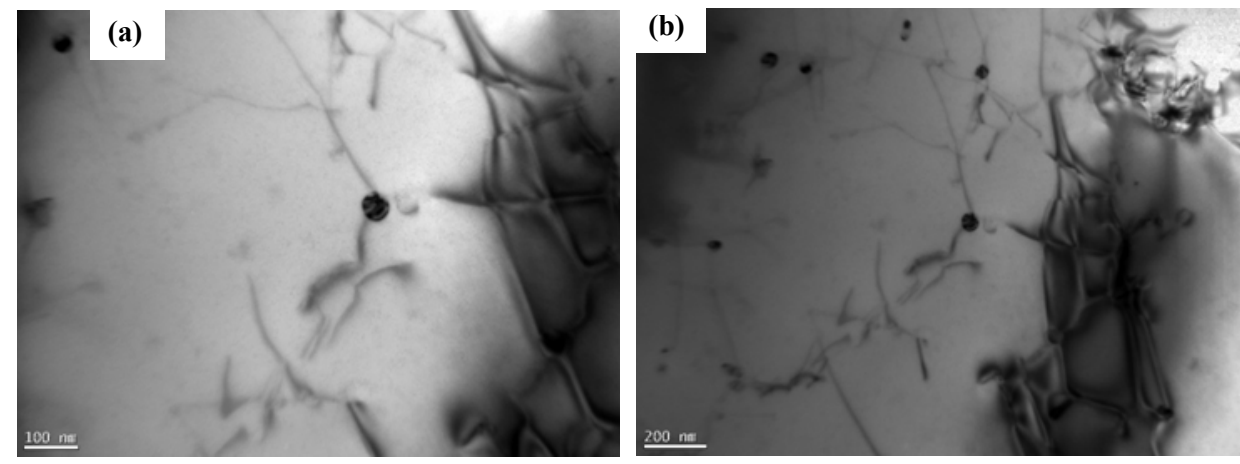

Fig. 62. Interaction of dislocations with carbides $\left(80 \mathrm{MPa}, 650^{\circ} \mathrm{C}\right)$. 
Figure 63 shows images from the uniformly elongated gauge area of a sample crept at 140 $\mathrm{MPa}$. When compared to Figure 61 from a sample crept at $80 \mathrm{MPa}$, a higher density of dislocations can be seen. The sub-grain size is smaller when compared to Figure 62 at $80 \mathrm{MPa}$ with regard to higher amount of time. Figures 63(a) and 63(d) show elongated grains and subgrains due to creep. In Figure 63(e) strain-free, dislocation-free small newly formed recrystallized grains can be seen. This supports the idea that recrystallization occurs under the effect of strain and temperature. Figures 63(f) and 63(g) show typical sub-grain boundaries formed by arrays of dislocations and the carbides present on the sub-grain boundaries. Figure 63(f) also shows small carbides and their interaction with dislocations. Figure 63 on the whole shows a mixture of sub-grains with free dislocations, dislocation-free sub-grains, recrystallized grains, and carbides interacting with sub-grain boundaries and dislocations and hence depicting a clear picture of recovery and recrystallization during the creep.
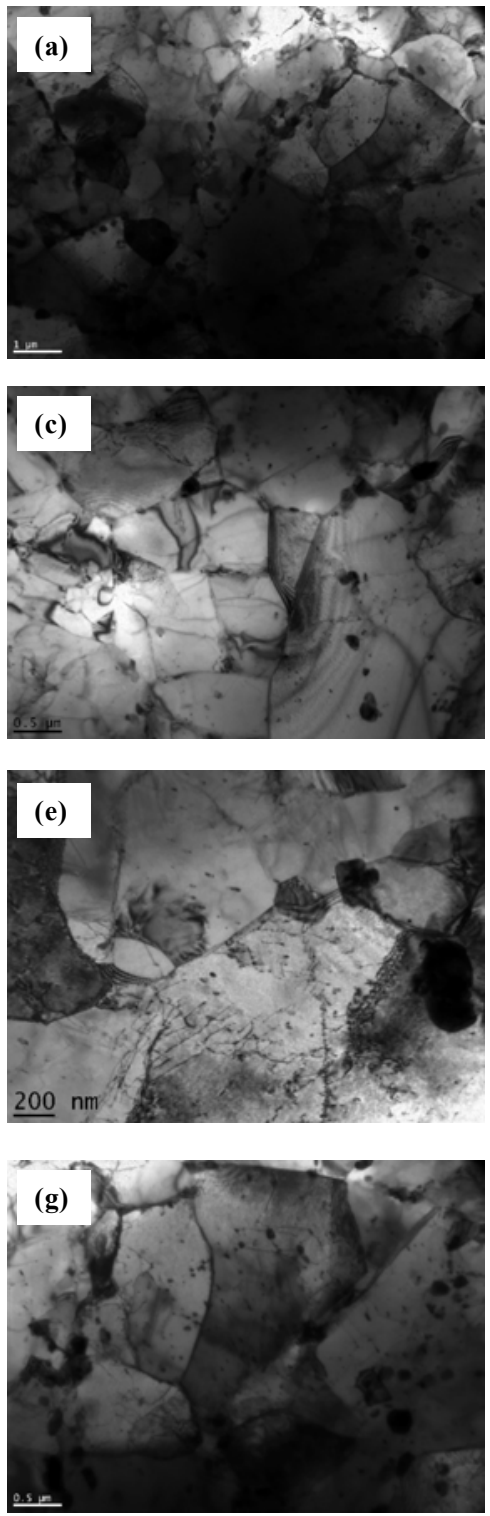
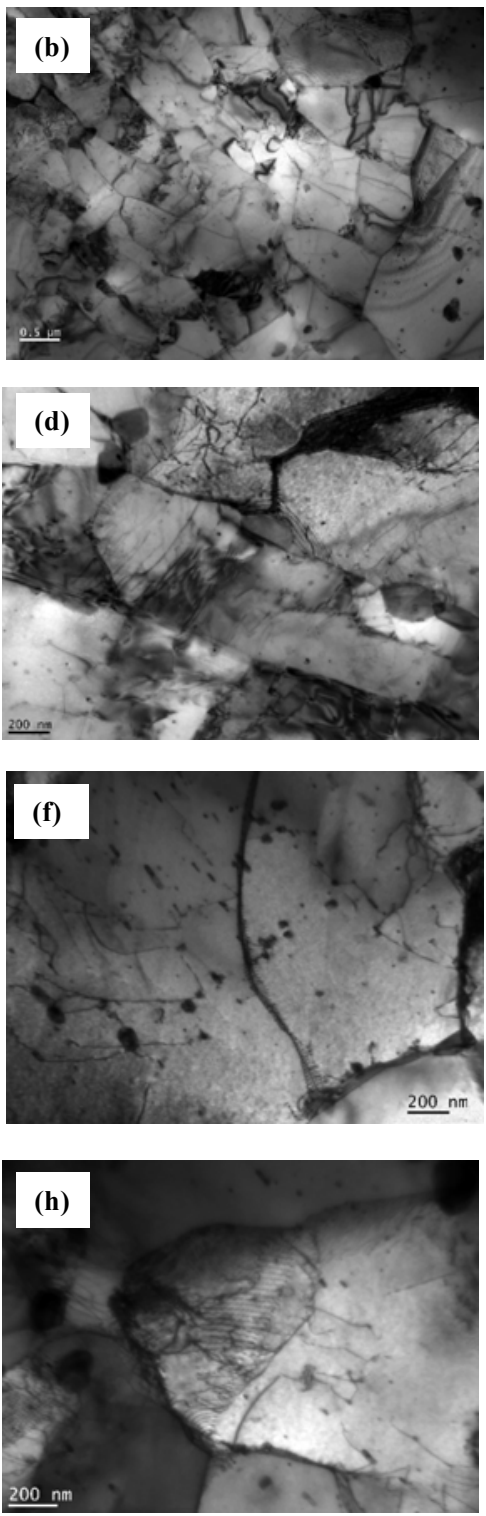

Fig. 63. TEM images from the gauge section after creep at $140 \mathrm{MPa}$. 
In conclusion, the main findings of our metallurgical investigation can be summarized as follows.

- A standard metallographic technique was developed for observing cavity and voids by multiple etching and polishing. Close to the fracture surface, the calculated values of the number density of voids and cavities are about $13,000 / \mathrm{mm}^{2}$ and $33,000 / \mathrm{mm}^{2}$ for samples tested at $120 \mathrm{MPa}$ and $140 \mathrm{MPa}$, respectively. Cavity density is increased by a factor of about 3 by increasing the stress level from 120 to $140 \mathrm{MPa}$. Further away from the fracture surface the number density of cavities is much lower.

- Creep voids/cavities form at grain boundaries and later they coalesce and become oriented along the grain boundary parallel to the stress axis, which leads to formation of grain boundary micro cracks. A number of creep voids/cavities coalesce and lead to dimples/larger cavities. This was mainly observed at $140 \mathrm{MPa}$.

- Carbides form mostly at grain boundaries, and martensite packet or lath boundaries. Carbides exhibit spherical morphology in the undeformed samples and the average size ranged from 50-90 nm. However, occasionally larger carbides around $200 \mathrm{~nm}$ are observed. Carbides are refined in the fracture area, i.e., in the region of highest strain. This could be because of the dissolution of carbides assisted by strain as well as recrystallization of the alpha phase. In the deformed areas carbides are located along grain boundaries and oriented parallel to the stress axis. Interestingly, carbides are deformed and elongated at high stress levels (140 MPa).

- At $650{ }^{\circ} \mathrm{C}$ and stress of $120 \mathrm{MPa}$, there is no evidence of the void formation and presence of any cracks until $75 \%$ of fracture time. Most of the damage initiation and accumulation occurs at the very end term of the creep process at this condition.

- The microstructure study at the necking part of the fractured samples at 125 and $140 \mathrm{MPa}$ clearly showed that no voids or cracks form until $30 \%$ creep deformation. This is almost after $99 \%$ of the whole creep life. These results show that the fracture mechanism is formation of voids in the vicinity of the deformed carbides that are located at grain boundaries and subjected to higher strain than 30\%. These voids grow very fast and coalesce resulting in micro grain boundary cracks and dimples or larger voids. Voids are oriented parallel to the stress axis and are not distributed randomly.

- Microstructure of the near-fracture surface region with the strain of around $40 \%$ clearly shows recrystallization, which is a very interesting phenomenon. EBSD images show very well that dislocation creep is the main deformation mechanism. Increasing strain during the creep test results in formation of more sub-grains and refinement in sub-grain size. The angles of these sub-grains tend to increase by increasing strain. The dynamic recrystallization mechanism could be continuous; however this needs a more detailed study. There are a couple of hypotheses at this time but it is clear that high amount of strain is applied to those regions of near-fracture surface which overcomes the barrier energy for recrystallization. The recrystallization itself could damp the strain energy of deformation, resulting in higher elongation or creep strain. It is worth mentioning that the average size and fraction of carbides show a change in this region.

- The microstructure study at the necking part of the fractured samples at $100 \mathrm{MPa}$ interestingly shows that micro-cavities form after about $25-35 \%$ creep deformation. This is still near $90 \%$ of whole creep life. These results show that by decreasing the level of stress, we could be able to observe the very first steps of void initiation. Also the fracture mechanism is formation of voids at the vicinity of the deformed carbides that are located 
at the grain boundaries and subjected to the higher strain. These voids grow rapidly into larger voids and link-up into microcracks.

- Carbide size ranges from $320 \mathrm{~nm}$ to $250 \mathrm{~nm}$ going from the fracture surface to the uniformly deformed area.

- Fairly large numbers of cavities are observable not only at the fracture surface but also at the deformed areas at the lower stress level of $80 \mathrm{MPa}$. However, cavity formation, coalescence and propagation occur only after $90 \%$ of the creep life even at these lower levels of stress.

- Formation and growth of sub-grain boundaries is the main microstructure evolution mechanism during creep at lower stress levels. The creep mechanism is still dislocation creep at stress levels around $80 \mathrm{MPa}$.

\section{Conclusions}

The feasibility of nondestructive evaluation (NDE) is dependent on the presence of perceivable precursors well before failure so that they can be detected by inspections during regular maintenance periods. The working hypothesis of our project was that this conventional mode of operation of NDE cannot be adapted to creep damage detection in 9Cr-1Mo steel because perceivable damage forms too close to rupture. This hypothesis was fully confirmed by our investigation. To facilitate creep assessment even before the appearance of significant material damage, we proposed a structural health monitoring (SHM) approach that avoids this problem by (i) continuously monitoring the state of the material at critical locations and (ii) being sensitive to the presence of plastic strain essentially from the beginning of creep in the primary stage.

The main goal of this project was the development and validation of a novel NDE technique for in-situ monitoring of ferritic-martensitic steels like Grade 91 9Cr-1Mo, which are candidate materials for Generation IV nuclear energy structural components operating at temperatures up to $\approx 650{ }^{\circ} \mathrm{C}$ and for steam generator tubing for sodium-cooled fast reactors. Successful assessment of thermo-mechanical damage requires a clear separation between thermally activated microstructural evolution and creep damage caused by simultaneous mechanical stress. First, we analyzed the feasibility of a directional ACPD probe with square-electrode configuration for in-situ monitoring of creep in high-temperature environments. By detecting variations in the ratio of the axial and lateral resistances, the sensor can distinguish directional creep damage from mostly non-directional thermally activated microstructural evolution. The main advantage of directional electric resistance measurements over non-directional ones is sensitivity to early stages of creep, which is still a challenge for most existing NDE methods. We found that under uniaxial stress square-electrode PD sensors exhibit a geometrical gauge factor of $G \approx 5$, i.e., more than twice that of a conventional strain gauge.

The sensitivity of the PD creep sensor to material effects was also investigated. In order to optimize the sensitivity of the probe to material damage, we used a square-electrode configuration which is much more directional than the common in-line configuration. However, the probe's sensitivity to creep-induced electric anisotropy is still relatively low, especially in materials with cubic symmetry that do not exhibit crystallographic electric anisotropy due to preferred grain orientation. In this way, the creep sensor is mostly sensitive to geometrical effects up to the initiation of preferentially oriented microcracks and the monitored resistance ratio is initially proportional to the creep strain.

Due to its high geometrical gauge factor, a permanently installed directional ACPD probe acts essentially as a high-temperature strain gauge throughout most of the creep life of the monitored component. In most cases, the influence of electric resistivity anisotropy caused by 
preferentially oriented material defects remains negligible until the last phase of secondary creep and becomes significant only in the tertiary stage of creep just before rupture.

Microstructure evolution and cavity nucleation and growth over the creep life of 9Cr-1Mo ferritic-martensitic steel were investigated. Microstructure of the samples was characterized by transmission electron microscopy (TEM) and by electron backscattered diffraction analysis (EBSD)/orientation imaging microscopy (OIM) and scanning electron microscopy (SEM) to determine the accurate grain size, boundary character, texture formation, and precipitation characteristics. The results showed that at high levels of stress damage initiation and failure occur in a very short time, whereas at lower stress levels damage initiation and propagation are distinguishable before fracture. As far as the developed NDE method is concerned, the main metallurgical findings are that creep voids/cavities are formed at the grain boundaries and sometimes they coalesce and become oriented along the grain boundary parallel to the stress axis, which leads to formation of oriented grain boundary micro cracks. At $650{ }^{\circ} \mathrm{C}$ and $120 \mathrm{MPa}$, there is no evidence of void formation and presence of any cracks until $75 \%$ of fracture time. Our microstructural study showed that at the necking part of samples crept at 125 and $140 \mathrm{MPa}$ voids or cracks are formed only above 30\% creep deformation, which corresponds to almost $99 \%$ of the whole creep life.

Our aim was to establish the most effective nondestructive materials evaluation technique for various degradation modes in Grade 91 steel as well as to provide the necessary mechanismbased underpinnings for relating the two. Based on our investigation, early creep monitoring is best achieved by a measurement of the associated strain. Our NDE development effort was based on the directional alternating current potential drop (DACPD) technique using a squareelectrode configuration. This measurement technique is based on a modified potential drop (PD) technique as it measures simultaneously two resistance values in orthogonal directions. Our tests showed that small anisotropic changes in the two resistances caused by directional effects of creep can be readily distinguished from potentially far larger isotropic changes caused by nondirectional reversible and irreversible thermal effects. The sensitivity of the square-electrode PD sensor to geometrical and material variations was analyzed separately and the analytical predictions were validated by experimental tests. The directional PD technique was found to exhibit high sensitivity that allows the detection of both elastic and plastic strains. Extensive creep tests lasting between 4 and 4,000 hours were conducted in Grade 91 9Cr-1Mo between 70 $\mathrm{MPa}$ and $150 \mathrm{MPa}$ at $650{ }^{\circ} \mathrm{C}$.

Our tests with permanently mounted electrodes demonstrated the feasibility of the DACPD technique for creep to monitoring. Such permanently mounted probes are ideal for both continuous and intermittent creep monitoring since the electrodes attached to the surface follow the deformation of the component and therefore the measured resistance ratio is sensitive not only to material changes caused by creep damage but also to much stronger geometrical changes caused directly by creep deformation. However, in some applications, it is desirable to use deployable probes even if the sensitivity to creep is necessarily lower. Therefore, we also developed a deployable DACPD probe that is solely sensitive to material effects and can be used to detect creep-induced electric anisotropy in the material by manually scanning the surface area of interest. Deployable probes with spring-loaded contact pins can be used to selectively assess the otherwise negligible material effects of creep. Because of the inevitably larger positioning error of spring-loaded electrodes, deployable probes exhibit is inherently higher measurement uncertainty than their permanently mounted counterparts. Still, such deployable probes might find application in nondestructive creep assessment because they can be used to scan larger areas 
of interest, including welds and the crucial boundary region between welds and the neighboring heat-affected zone of the base metal.

\section{References}

1. Maruyama K, Sawada K, and Koike J, ISIJ Int 41, 641-653 (2001).

2. Sposito G, Ward C, Cawley P, Nagy PB, and Scruby C, NDT\&E Int 43, 555-567 (2010).

3. Furtado HC, de Almeida LH, Le May I, Mat Char 58, $72-77$ (2007).

4. Perry AJ, J Mat Sci 9, 1016-1039 (1974).

5. Jeong H, Kim D-H, Mat Sci Eng A 337, 82-87 (2002).

6. Andreikiv OE, Lesiv RM, Levyts'ka NM, Mat Sci 45, 11-17 (2009).

7. Betten J, Sklepus S, Zolochevsky A, Eng Fract Mech 59, 623-641 (1998).

8. Fuji A, Kitagawa M, Yokobori AT, Eng Fract Mech 45, 39-50 (1993).

9. Dogan B, Nikbin K, Petrovski B, Ceyhan U, Dean DW, Int J Press Vessels Pip 83, 784-797 (2006).

10. Sukekawa M, Shiga M, Tobita Y, Kirihara S, Int Conf Creep, JSME, 321-325 (1986).

11. Kirihara S, Shiga M, Sukekawa M, Yoshioka T, Asano C, J Soc Mat Sci 33, 1097-1102 (1984).

12. Yu KM, Nahm SH, Kim YI, J Mat Sci Lett 18, 1175-1176 (1999).

13. Nahm SH, Yu KM, Park JS, Nam YH, Han SI, Kim JM, Int J Mod Phys B 17, 1615-1620 (2003).

14. Nahm SH, Kim JM, Park JS, Yu KM, Kim DK, Kim A, Key Eng Mat 270, 1212-1217 (2004).

15. Nagy PB, Insight 52, 61-71 (2010).

16. Masuyama F, Int J Press Vess Piping 83, 819-825 (2006).

17. Sukegawa M, Shiga M, Asano C, Tobita Y, Kirihara S, J Soc Mat Sci 34, 1478-1482 (1985).

18. Bressers J, Hessler W, Hildebrandt UW, Willems H, in High Temperature Materials for Power Engineering (Kluwer, 1990) pp. 629-642.

19. Willems H, Dobmann G, Nucl Eng Design 128, 139-149 (1991).

20. Masuyama F, Tokunaga T, Shimohata N, Yamamoto T, Hirano M, in Creep \& Fracture in High Temperature Components: Design \& Life Assessment Issues (DEStech Publications, 2009) pp. 19-30.

21. Pelloux RM, Peltier JM, Zilberstein VA, J Eng Mat Techn 111, 19-20 (1989).

22. Bowler N, Meas Sci Techn 22, 012001 (2011).

23. Madhi E, Sposito G, Davies CM, Cawley P, Nagy PB, in Rev Progr Quant NDE 30, 16311638 (2011).

24. Madhi E, Nagy PB, in Rev Progr Quant NDE 29, 1325-1332 (2010).

25. Madhi E, Nagy PB, in Rev Progr Quant NDE 30, 1233-1240 (2011).

26. Madhi E, Nagy PB, in Rev Progr Quant NDE 30, 1623-1630 (2011).

27. Madhi E, Nagy PB, NDT\&E Int 44, 708-717 (2011).

28. Berezin VV, Kostyurin AA, Popov YA, J Eng Phys Thermophys 54, 197-201 (1988).

29. Das UC, Li P, J Appl Geophys 35, 63-67 (1996).

30. Tatarnikov VM, Meas Tech 13, 877-881 (1970).

31. Yu F, Nagy PB, J Nondestr Eval 24, 143-52 (2005).

32. Shafiro B, Kachanov M, J Appl Phys 87, 8561-8569 (2000).

33. Viswanathan R, Stringer J, J Eng Mat Techn 122, 246-255 (2000).

34. Sedman KG, Thornley JC, Griffin RM, Eng Fail Anal 4, 89-98 (1997).

35. Budden J, Int J Press Ves Piping 75, 509-519 (1998). 
36. Ellis F, Viswanathan R, Fitness for Service Evaluation in Petroleum and Fossil Plants, ASME (1998) pp. 59-76.

37. Francis JA, Mazur W, Bhadeshia HK, Mat Sci Techn 22, 1387-1395 (2006).

38. Hongo H, Tabuchi M, Takahashi Y, J Solid Mech Mat Eng 3, 464-469 (2009).

39. Venkatasubramanian TV, Unvala BA, J Phys E Sci Instr 17, 765-771 (1984).

40. Dover WD, Collins R, Michael DH, Phil Trans Roy Soc Lond A 320, 271-283 (1986).

41. Michael D, Waechter R, Collins R, Roy Soc Proc London A 381, 139-157 (1982).

42. Saguy H, Rittel D, NDT\&E Int 40, 505-509 (2007).

43. Bowler N, Huang Y, IEEE Trans Magn 41, 2102-2110 (2005).

44. Bowler JR, Bowler N, Proc Roy Soc Lond A 463, 817-836 (2007).

45. Hwang S, Ballinger RG, Meas Sci Techn 3, 62-74 (1992).

46. Dover WD, Charlesworth FDW, Taylor KA, Collins R, Michael DH, in The Measurement of Crack Length and Shape During Fracture and Fatigue, Warley, (1980) pp. 222-260.

47. Mirshekar-Syahkal D, Collins R, Michael DH, J Nondestr Eval 3, 65-76 (1982).

48. Blodgett M, Nagy PB, Appl Phys Lett 72, 1045-1047 (1998).

49. Tsang T, Classical Electrodynamics, World Scientific (1997) pp. 59-62.

50. Prajapati S, Nagy PB, Cawley P, NDT\&E Int 47, 56-65 (2012).

51. Williams RK, Graves RS, McElroy DL, Int J Thermophys 5, 301-313 (1984).

52. Swindeman RW, Santella ML, Maziasz PJ, Roberts BW, Coleman K, Int J Press Vess Piping 81, 507-512 (2004).

53. Klueh RL, Int Mat Rev, 50, 287-310 (2005).

54. Murty KL, Charit I, J Nucl Mat 383, 189-195 (2008).

55. Vitek JM, Klueh RL, Metall Trans A 14, 1047-1055 (1983).

56. Chandravathi KS, Laha K, Parameswaran P, Mathew MD, Int J Press Vess Piping 89, 162169 (2012).

57. Jones WB, Hills CR, Polonis DH, Metall Trans A 22, 1049-1058 (1991).

58. Sonderegger B, Mitsche S, Cerjak H, Mat Sci Eng A 48, 466-470 (2008).

59. Chen RP, Ghassemi-Armaki H, Maruyama K, Igarashi M, Mat Sci Eng A 528, 4390-4394 (2011).

60. Anderson P, Bellgardt T, Jones FL, Mat Sci Techn 19, 207-213 (2003).

61. Cerri E, Evangelista E, Spigarelli S, Bianchi P, Mat Sci Eng A 245, 285-292 (1998). 